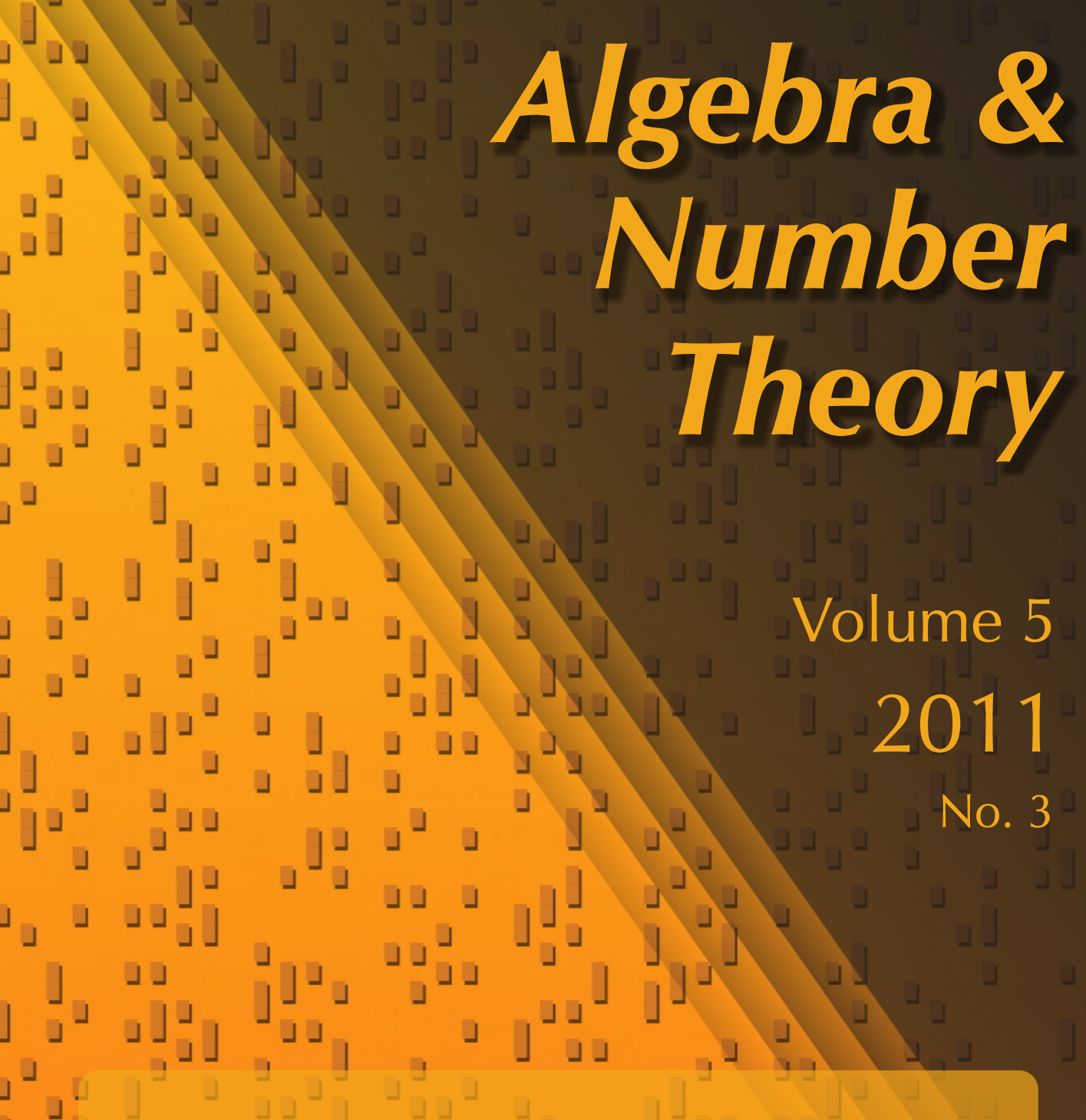

A categorical proof of the Parshin reciprocity laws on algebraic surfaces

Denis Osipov and Xinwen Zhu

\lrcorner \lrcorner \lrcorner$\lrcorner$

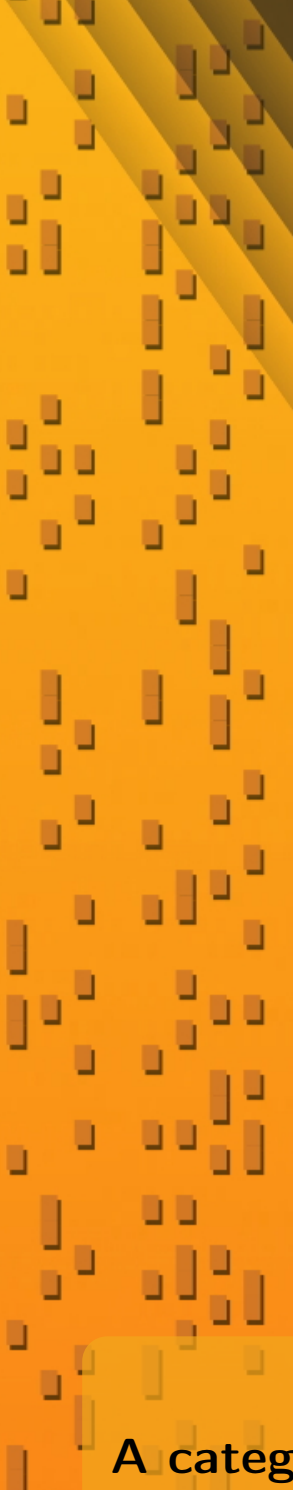




\title{
A categorical proof of the Parshin reciprocity laws on algebraic surfaces
}

\section{Denis Osipov and Xinwen Zhu}

\begin{abstract}
We define and study the 2-category of torsors over a Picard groupoid, a central extension of a group by a Picard groupoid, and commutator maps in this central extension. Using this in the context of two-dimensional local fields and twodimensional adèle theory we obtain the two-dimensional tame symbol and a new proof of Parshin reciprocity laws on an algebraic surface.
\end{abstract}

\section{Introduction}

Let $C$ be a projective algebraic curve over a perfect field $k$. The famous Weil reciprocity law states that

$$
\prod_{p \in C} \mathrm{Nm}_{k(p) / k}\{f, g\}_{p}=1
$$

where $f, g \in k(C)^{\times}$,

$$
\{f, g\}_{p}=(-1)^{v_{p}(f) v_{p}(g)} \frac{f^{v_{p}(g)}}{g^{v_{p}(f)}}(p)
$$

is the one-dimensional tame symbol, and $k(p)$ is the residue field of the point $p$. The product (1-1) contains only finitely many terms not equal to 1 .

There is a proof of this law (and the analogous reciprocity law for residues of rational differential forms: sum of residues equals to zero) by reduction to the case of $\mathbf{P}_{k}^{1}$ using the connection between tame symbols (and residues of differentials) in extensions of local fields; see, for example, [Serre 1988, Chapters 2 and 3].

On the other hand, Tate [1968] gave a definition of the local residue of a differential form as the trace of a certain infinite-dimensional matrix. Starting from this definition he gave an intrinsic proof of the residue formula on a projective algebraic curve $C$ using the fact that $\operatorname{dim}_{k} H^{i}\left(C, O_{C}\right)<\infty$, for $i=0,1$.

Osipov was financially supported by Russian Foundation for Basic Research (grant no. 11-01-00145) and by the Programme for the Support of Leading Scientific Schools of the Russian Federation (grant no. NSh-4713.2010.1). Zhu's research is supported by NSF grant under DMS-1001280.

MSC2000: primary 19F15; secondary 18D05.

Keywords: Picard groupoids, central extensions, commutator maps, two-dimensional local fields, higher adeles, reciprocity laws. 
The multiplicative analog of Tate's approach, i.e., the case of the tame symbol and the proof of Weil reciprocity law, was done later by Arbarello, De Concini and Kac [1988]. They used the central extension of the infinite-dimensional group $\mathrm{GL}(K)$ of continuous automorphisms of $K$, where $K=k((t))$, by the group $k^{\times}$, and obtained the tame symbol up to sign as the commutator of the lifting of two elements from $K^{\times} \subset \mathrm{GL}(K)$ to this central extension. Hence, as in Tate's proof mentioned above, they obtained an intrinsic proof of the Weil reciprocity law on an algebraic curve. However, in this proof the exterior algebra of finite-dimensional $k$-vector spaces was used. Therefore difficult sign conventions were used in this paper to obtain the reciprocity law. To avoid these difficulties, Beilinson, Bloch and Esnault [2002] used the category of graded lines instead of the category of lines. The category of graded lines has nontrivial commutativity constraints multipliers $(-1)^{m n}$, where $m, n \in \mathbb{Z}$ are corresponding gradings. In other words, they used the Picard groupoid of graded lines which is a nonstrictly commutative instead of strictly commutative Picard groupoid. It was the first application of this notion of nonstrictly commutative Picard groupoid.

Now let $X$ be an algebraic surface over a perfect field $k$. For any pair $x \in C$, where $C \subset X$ is a curve that $x \in C$ is a closed point, it is possible to define the ring $K_{x, C}$ such that $K_{x, C}$ is isomorphic to the two-dimensional local field

$$
k(x)((t))((s))
$$

when $x$ is a smooth point on $C$ and $X$. If $x$ is not a smooth point, then $K_{x, C}$ is a finite direct sum of two-dimensional local fields (see Section 5B of this paper). For any two-dimensional local field $k^{\prime}((t))((s))$ one can define the two-dimensional tame symbol of 3 variables with values in $k^{\prime \times}$, see Section 4A and [Parshin 1975, 1984, §3]. Parshin formulated and proved the reciprocity laws for two-dimensional tame symbols, but his proof was never published. Contrary to the one-dimensional case, there are a lot of reciprocity laws for two-dimensional tame symbols, which belong to two types. For the first type we fix a point on the surface and will vary irreducible curves containing this point. For the second type we fix a projective irreducible curve on the surface and will vary points on this curve. Parshin's idea for the proof, for example, of more unexpected first type of reciprocity laws, was to use the chain of successive blowups of points on algebraic surfaces. Later, Kato [1986, Proposition 1] generalized the reciprocity laws for excellent schemes by using the reduction to the reciprocity law of Bass and Tate for Milnor K-groups of some field $L(t)$. He used them to construct an analog of the Gersten-Quillen complex for Milnor K-theory.

In this paper, we give a generalization of Tate's proof of the reciprocity law on an algebraic curve to the case of two-dimensional tame symbols and obtain an 
intrinsic proof of Parshin reciprocity laws for two-dimensional tame symbols on an algebraic surface.

To fulfill this goal, we first generalize the notion of a central extension of a group by a commutative group and of the commutator map associated to the central extension. More precisely, we define and study in some detail the properties of the category of central extensions of a group $G$ by a (nonstrictly commutative) Picard groupoid $\mathscr{P}$. Roughly speaking, an object in this category is a rule to assign every $g \in G$ a $\mathscr{P}$-torsor, satisfying certain properties. For such a central extension $\mathscr{L}$ we define a map $C_{3}^{\mathscr{L}}$ which is an analog of the commutator map. In this case when $G$ is abelian, this commutator map is an antisymmetric and trimultiplicative map from $G^{3}$ to the group $\pi_{1}(\mathscr{P})$. Let us remark that to obtain some of these properties, we used the results of Breen [1999] on group-like monoidal 2-groupoids. We hope these constructions will be of some independent interest.

We then apply this formalism to $\mathscr{P}=\mathscr{P} i c^{\mathbb{Z}}$, where $\mathscr{P} i c^{\mathbb{Z}}$ stands for the Picard groupoid of graded lines. The key ingredient here is Kapranov's [2001] gradeddeterminantal theory, which associates a $\mathscr{P} i c^{\mathbb{Z}}$-torsor to every 1 -Tate vector space (a locally linearly compact vector space). This allows one to construct the central extension Det of GL( $\mathbb{K}$ ) by $\mathscr{P} i c^{\mathbb{Z}}$, where $\mathbb{K}$ is a two-dimensional local field (or more generally, a 2-Tate vector space). It turns out that the two-dimensional tame symbol coincides with the commutator map $C_{3}^{\text {Det }}$. Finally, using "semilocal" adèle complexes on an algebraic surface we obtain that the corresponding central extension constructed by semilocal fields on the surface is the trivial one. This leads us to a new proof of Parshin's reciprocity laws on an algebraic surface, which is distinct from both Parshin's original approach as well as Kato's.

Our approach to the reciprocity laws on the algebraic surfaces has the following features. First, we use the nonstrictly commutative Picard groupoid, which can be regarded as another application of this notion after [Beilinson et al. 2002]. However, unlike the one-dimensional case where one can just plays with the usual Picard groupoid of lines (though complicated, as done in [Arbarello et al. 1988]), the use of $\mathscr{P} i c^{\mathbb{Z}}$ is essential here. This indicates that the nonstrictly commutative Picard groupoid is an important and fruitful mathematical object that deserves further attention. Also, in order to apply this notion, we develop certain constructions in higher categories (e.g., the commutator map $C_{3}^{\mathscr{L}}$ ), which could be potentially useful elsewhere. Second, as in the one-dimensional case, our approach uses a local-toglobal (in other words, factorization) principle. Since the local-to-global (factorization) principle in the one-dimensional story is very important in the Langlands program and conformal field theory, we hope our approach is just a shadow of a whole fascinating realm of mathematics yet to be explored. Finally, our approach can be generalized by replacing the ground field $k$ by an Artinian ring $A$ (and even more general rings) and we can obtain reciprocity laws for two-dimensional 
Contou-Carrère symbols. By choosing $A$ appropriately, this specializes to residue formulas for algebraic surfaces. ${ }^{1}$ We will carefully discuss this in a future paper.

The paper is organized as follows. In Section 2 we describe some categorical constructions, which we need further on. In Section 2A we recall the definition of a Picard groupoid. In Section 2B we discuss the difference between strictly commutative and nonstrictly commutative Picard groupoids. In Section $2 \mathrm{C}$ we describe the 2-category of $\mathscr{P}$-torsors, where $\mathscr{P}$ is a Picard groupoid. In Section 2D we study the Picard groupoid of homomorphisms from a group $G$ to a Picard groupoid $\mathscr{P}$ and describe the "commutator" of two commuting elements from $G$ with values in $\pi_{1}(\mathscr{P})$. In Section $2 \mathrm{E}$ we define and study the Picard 2-groupoid of central extensions of a group $G$ by a Picard groupoid $\mathscr{P}$. We define and study properties of the commutator category of such a central extension, and finally study the "commutator" of three commuting elements form $G$ with values in $\pi_{1}(\mathscr{P})$. This section may be of independent interest.

In Section 3 we recall the theory of graded-determinantal theories on Tate vector spaces. We recall the definition and basic properties of the category of $n$-Tate vector spaces in Section 3A. In Section 3B we recall the definition of determinant functor from the exact category (Tate ${ }_{0}$, isom) to the Picard groupoid $\mathscr{P} i c^{\mathbb{Z}}$ of graded lines and the definition of graded-determinantal theory on the exact category Tate $_{1}$ of 1-Tate vector spaces.

In Section 4 we apply the constructions given above to one-dimensional and two-dimensional local fields. In Section 4A we review one-dimensional and twodimensional tame symbols. In Section 4B we obtain a description of the onedimensional (usual) tame symbol as some commutator. In Section $4 \mathrm{C}$ we obtain the two-dimensional tame symbol as commutator of 3 elements in some central extension of the group $K^{\times}=k((t))((s))^{\times}$by the Picard groupoid $\mathscr{P} i c^{\mathbb{Z}}$.

In Section 5 we obtain the reciprocity laws. In Section 5A we give the proof of Weil reciprocity law using the constructions given above and adèle complexes on a curve. In Section 5B we apply the previous results in order to obtain a proof of Parshin's reciprocity laws on an algebraic surface using "semilocal" adèle complexes on an algebraic surface.

\section{General nonsense}

2A. Picard groupoid. Let $\mathscr{P}$ be a Picard groupoid, i.e., a symmetric monoidal group-like groupoid. Let us recall that this means that $\mathscr{P}$ is a groupoid, together with a bifunctor

$$
+: \mathscr{P} \times \mathscr{P} \rightarrow \mathscr{P}
$$

${ }^{1}$ The generalization of Tate's approach to the $n$-dimensional residue of differential forms was done in [Beilinson 1980], but that note contains no proofs. 
and natural (functorial) isomorphisms

$$
a_{x, y, z}:(x+y)+z \simeq x+(y+z)
$$

called the associativity constraints, and natural (functorial) isomorphisms

$$
c_{x, y}: x+y \simeq y+x
$$

called the commutativity constraints, such that:

(i) For each $x \in \mathscr{P}$, the functor $y \mapsto x+y$ is an equivalence.

(ii) The pentagon axiom holds, i.e., the following diagram is commutative:

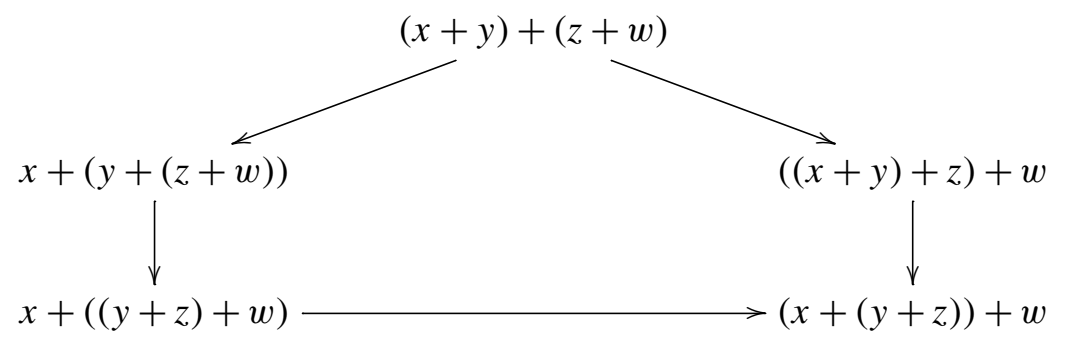

(iii) The hexagon axiom holds, i.e., the following diagram is commutative:

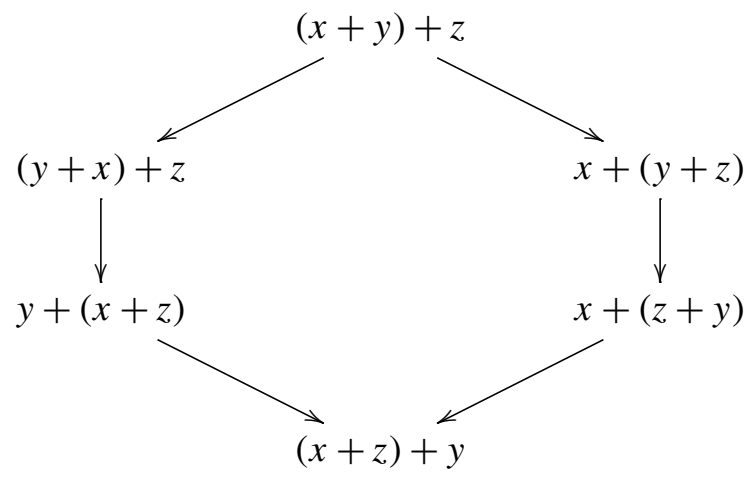

(iv) For any $x, y \in \mathscr{P}, c_{y, x} c_{x, y}=i d_{x+y}$.

A unit $(e, \varphi)$ of $\mathscr{P}$ is an object $e \in \mathscr{P}$ together with an isomorphism $\varphi: e+e \simeq e$. It is an exercise to show that $(e, \varphi)$ exists and is unique up to a unique isomorphism. For any $x \in \mathscr{P}$, there is a unique isomorphism $e+x \simeq x$ such that the following diagram is commutative:

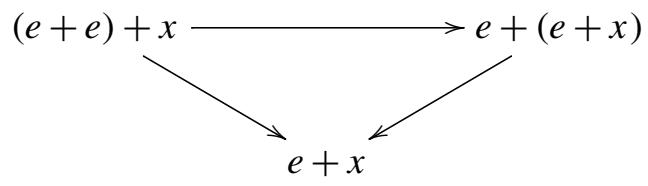


and therefore $x+e \simeq e+x \simeq x$. For any $x \in \mathscr{P}$, we choose an object, denoted by $-x$, together with an isomorphism $\phi_{x}: x+(-x) \simeq e$. The pair $\left(-x, \phi_{x}\right)$ is called an inverse of $x$, and it is unique up to a unique isomorphism. We choose for each $x$ its inverse $\left(-x, \phi_{x}\right)$, then we have a canonical isomorphism

$$
\begin{aligned}
-(-x) & \simeq e+(-(-x)) \simeq(x+(-x))+(-(-x)) \\
& \simeq x+((-x)+(-(-x))) \simeq x+e \simeq x,
\end{aligned}
$$

and therefore a canonical isomorphism

$$
(-x)+x \simeq(-x)+(-(-x)) \simeq e .
$$

Observe that we have another isomorphism $(-x)+x \simeq x+(-x) \simeq e$ using the commutativity constraint. When the Picard groupoid $\mathscr{P}$ is strictly commutative (Section 2B), these two isomorphisms are the same [Zhu 2009, Lemma 1.6], but in general they are different.

If $\mathscr{P}_{1}, \mathscr{P}_{2}$ are two Picard groupoids, then $\operatorname{Hom}\left(\mathscr{P}_{1}, \mathscr{P}_{2}\right)$ is defined as follows. Objects are 1-homomorphisms, i.e., functors $F: \mathscr{P}_{1} \rightarrow \mathscr{P}_{2}$ together with isomorphisms $F(x+y) \simeq F(x)+F(y)$ such that the following diagrams are commutative:

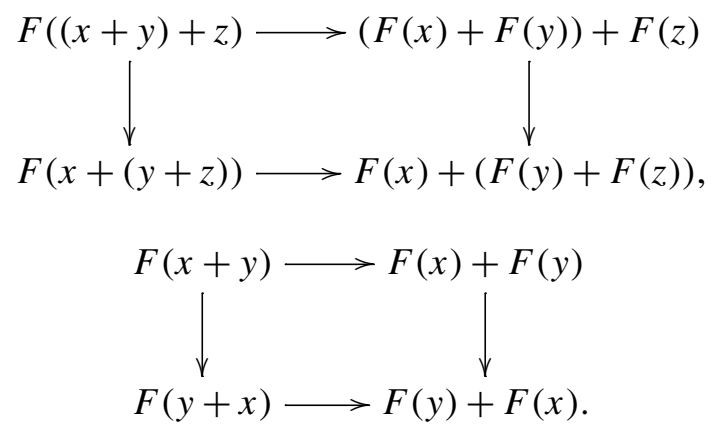

Morphisms in $\operatorname{Hom}\left(\mathscr{P}_{1}, \mathscr{P}_{2}\right)$ are 2-isomorphisms, i.e., natural transformations

$$
\theta: F_{1} \rightarrow F_{2}
$$

such that the following diagram is commutative:

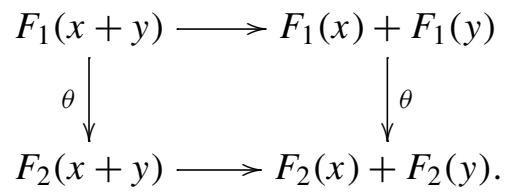

It is clear that $\operatorname{Hom}\left(\mathscr{P}_{1}, \mathscr{P}_{2}\right)$ has a natural structure as a Picard groupoid. Namely,

$$
\left(F_{1}+F_{2}\right)(x):=F_{1}(x)+F_{2}(x),
$$


and the isomorphism $\left(F_{1}+F_{2}\right)(x+y) \simeq\left(F_{1}+F_{2}\right)(x)+\left(F_{1}+F_{2}\right)(y)$ is the unique one such that the following diagram is commutative:

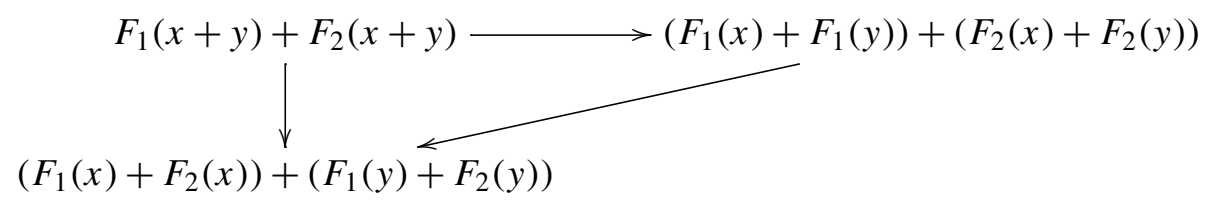

The associativity constraints and the commutativity constraints for $\operatorname{Hom}\left(\mathscr{P}_{1}, \mathscr{P}_{2}\right)$ are clear. If $\mathscr{P}_{1}, \mathscr{P}_{2}, \mathscr{P}_{3}$ are three Picard groupoids, then $\operatorname{Hom}\left(\mathscr{P}_{1}, \mathscr{P}_{2} ; \mathscr{P}_{3}\right)$ is defined as $\operatorname{Hom}\left(\mathscr{P}_{1}, \operatorname{Hom}\left(\mathscr{P}_{2}, \mathscr{P}_{3}\right)\right)$, called the Picard groupoid of bilinear homomorphisms from $\mathscr{P}_{1} \times \mathscr{P}_{2}$ to $\mathscr{P}_{3}$. The Picard groupoid of trilinear homomorphisms from $\mathscr{P}_{1} \times \mathscr{P}_{2} \times \mathscr{P}_{3}$ to $\mathscr{P}_{4}$ is defined similarly.

For a (small) monoidal group-like groupoid (or gr-category) $C$ we denote by $\pi_{0}(C)$ the group ${ }^{2}$ of isomorphism classes of objects. We denote by $\pi_{1}(C)$ the group $\operatorname{Aut}_{C}(e)$, where $e$ is the unit object of $C$. It follows that $\pi_{1}(C)$ is an abelian group. If $C$ is a Picard groupoid, then $\pi_{0}(C)$ is also an abelian group.

2B. Strictly commutative vs. nonstrictly commutative Picard groupoids. If the commutativity constraints $c$ further satisfy $c_{x, x}=\mathrm{id}$, then the Picard groupoid $\mathscr{P}$ is called strictly commutative. It is a theorem of Deligne's [1973] that the 2-category of strictly commutative Picard groupoids is 2-equivalent to the 2-category of 2term complexes of abelian groups concentrated on degree -1 and 0 , whose terms of degree -1 are injective abelian groups. ${ }^{3}$

Example 2.1. The most famous example is $\mathscr{P}=B A$, where $A$ is an abelian group, and $B A$ is the category of $A$-torsors. The tensor products of $A$-torsors make $B A$ a strictly commutative Picard groupoid. The 2-term complex of abelian groups that represents $B A$ under Deligne's theorem is any injective resolution of $A[1]$. If $A=k^{\times}$is the group of invertible elements in a field $k$, then $B A$ is also denoted by $\mathscr{P} i c$, which is the symmetric monoidal category of one-dimensional $k$-vector spaces.

However, it is also important for us to consider nonstrictly commutative Picard groupoids. The following example of a nonstrictly commutative Picard groupoid is crucial.

Example 2.2. Let $\mathscr{P} i c^{\mathbb{Z}}$ denote the category of graded lines (one-dimensional $k$ vector spaces with gradings) over a base field $k$. An object in $\mathscr{P} i c^{\mathbb{Z}}$ is a pair $(\ell, n)$, where $\ell$ is a one-dimensional $k$-vector space, and $n$ is an integer. The morphism set $\operatorname{Hom}_{\mathscr{P} i c^{\mathbb{Z}}}\left(\left(\ell_{1}, n_{1}\right),\left(\ell_{2}, n_{2}\right)\right.$ is empty unless $n_{1}=n_{2}$, and in this case, it is just

${ }^{2}$ The group structure on $\pi_{0}(C)$ is induced by the monoidal structure of $C$.

${ }^{3}$ In fact, Deligne's theorem holds in any topos. 
$\operatorname{Hom}_{k}\left(\ell_{1}, \ell_{2}\right) \backslash 0$. Observe that as a groupoid, $\mathscr{P} i c^{\mathbb{Z}}$ is not connected. In fact $\pi_{0}(\mathscr{P}) \simeq \mathbb{Z}$. The tensor product $\mathscr{P} i c^{\mathbb{Z}} \times \mathscr{P} i c^{\mathbb{Z}} \rightarrow \mathscr{P} i c^{\mathbb{Z}}$ is given as

$$
\left(\ell_{1}, n_{1}\right) \otimes\left(\ell_{2}, n_{2}\right) \mapsto\left(\ell_{1} \otimes \ell_{2}, n_{1}+n_{2}\right) .
$$

There is a natural associativity constraint that makes $\mathscr{P} i c^{\mathbb{Z}}$ a monoidal groupoid.

Convention. For the Picard groupoids $\mathscr{P} i c$ and $\mathscr{P} i c^{\mathbb{Z}}$, we will often use in this article the usual notation $\otimes$ for monoidal structures in these categories, although for a general Picard groupoid we denoted it as + .

We note that the commutativity constraint in category $\mathscr{P} i c^{\mathbb{Z}}$ is the interesting one. Namely,

$$
c_{\ell_{1}, \ell_{2}}:\left(\ell_{1} \otimes \ell_{2}, n_{1}+n_{2}\right) \simeq\left(\ell_{2} \otimes \ell_{1}, n_{2}+n_{1}\right), \quad c_{\ell_{1}, \ell_{2}}(v \otimes w)=(-1)^{n_{1} n_{2}} w \otimes v .
$$

Of course, there is another commutativity constraint on the category of graded lines given by $c(v \otimes w)=w \otimes v$. Then as a Picard groupoid with this naive commutativity constraints, it is just the strictly commutative Picard groupoid $\mathscr{P} i c \times$ $\mathbb{Z}$. There is a natural monoidal equivalence $\mathscr{P} i c^{\mathbb{Z}} \simeq \mathscr{P} i c \times \mathbb{Z}$, but this equivalence is not symmetric monoidal (that is, it is not a 1-homomorphism of Picard groupoids). We denote by

$$
F_{\mathscr{P} i c}: \mathscr{P i c} \mathbb{\mathbb { Z }}^{\rightarrow} \mathscr{P i c}
$$

the natural monoidal functor.

The importance of $\mathscr{P} i c^{\mathbb{Z}}$ lies in the following observation. Let us make the following convention.

Convention. For any category $\mathscr{C}$ we denote by $(\mathscr{C}$, isom) a category with the same objects as in the category $\mathscr{C}$, and morphisms in the category $(\mathscr{C}$, isom) are the isomorphisms in the category $\mathscr{C}$.

Now let Tate $_{0}$ be the category of finite dimensional vector spaces over a field $k$. The categories Tate $_{0}$ and (Tate $e_{0}$, isom) are symmetric monoidal categories under the direct sum. The commutativity constraints in the categories Tate $\mathrm{T}_{0}$ and ( Tate $_{0}$, isom) are defined in the natural way. Namely, the map $c_{V, W}: V \oplus W \rightarrow$ $W \oplus V$ is given by $c_{V, W}(v, w)=(w, v)$. Then there is a natural symmetric monoidal functor

$$
\operatorname{det}:\left(\text { Tate }_{0}, \text { isom }\right) \rightarrow \mathscr{P i c} c^{\mathbb{Z}}
$$

which assigns to every $V$ its top exterior power and the grading $\operatorname{dim} V$, the dimension of the vector space $V$ over the field $k$. Observe, however, that the functor $F_{\mathscr{P} i c} \circ$ det $:\left(\right.$ Tate $_{0}$, isom) $\rightarrow \mathscr{P}$ ic is not symmetric monoidal. 
It is a folklore theorem that the category of Picard groupoids (not necessarily strictly commutative) is equivalent to the category of spectra whose only nonvanishing homotopy groups are $\pi_{0}$ and $\pi_{1}{ }^{4}$. For example, $\mathscr{P} i c^{\mathbb{Z}}$ should correspond to the truncation $\tau_{\leq 1} \mathscr{K}$, where $\mathscr{K}$ is the spectra of algebraic $K$-theory of $k$.

2C. $\mathscr{P}$-torsors. Let $\mathscr{P}$ be a Picard groupoid. Recall (see also [Beilinson et al. 2002, Appendix A6] and [Drinfeld 2006, §5.1]) that a $\mathscr{P}$-torsor $\mathscr{L}$ is a module category over $\mathscr{P}$, i.e., there is a bifunctor

$$
+: \mathscr{P} \times \mathscr{L} \rightarrow \mathscr{L}
$$

together with natural isomorphisms

$$
a_{x, y, v}:(x+y)+v \simeq x+(y+v), \quad x, y \in \mathscr{P}, v \in \mathscr{L},
$$

satisfying

(i) the pentagon axiom, i.e., a diagram similar to (2-1) holds;

(ii) for any $x \in \mathscr{P}$, the functor from $\mathscr{L}$ to $\mathscr{L}$ given by $v \mapsto x+v$ is an equivalence;

(iii) for any $v \in \mathscr{L}$, the functor from $\mathscr{P}$ to $\mathscr{L}$ given by $x \mapsto x+v$ is an equivalence of categories.

It is clear that we can verify the condition (ii) of this definition only for the unit object $e$ of $\mathscr{P}$.

For any $v \in \mathscr{L}$, there is a unique isomorphism $e+v \simeq v$ such that the following diagram is commutative:

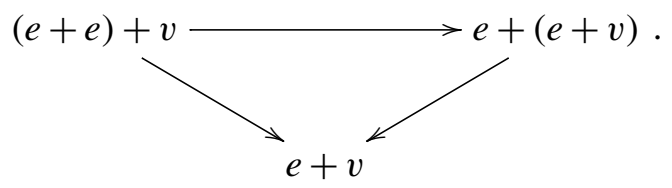

If $\mathscr{L}_{1}, \mathscr{L}_{2}$ are $\mathscr{P}$-torsors, then $\operatorname{Hom}_{\mathscr{P}}\left(\mathscr{L}_{1}, \mathscr{L}_{2}\right)$ is the category defined as follows. Objects are 1-isomorphisms, i.e., equivalences $F: \mathscr{L}_{1} \rightarrow \mathscr{L}_{2}$ together with isomorphisms $\lambda: F(x+v) \simeq x+F(v)$ such that the following diagram is commutative:

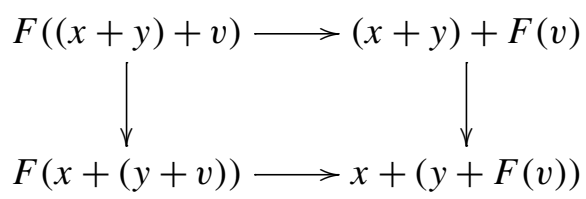

\footnotetext{
${ }^{4}$ Indeed, consider the geometrization of the nerve of $\mathscr{P}$. Then the Picard structure of $\mathscr{P}$ puts an $E_{\infty}$-structure on this space.
} 
Morphisms are natural transformations $\theta: F_{1} \rightarrow F_{2}$ such that the following diagram is commutative:

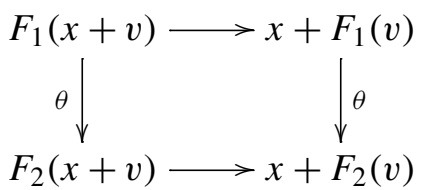

From these discussions it follows that all $\mathscr{P}$-torsors form a 2-category, denoted by $B \mathscr{P}$. We will choose, once and for all, for any $\mathscr{P}$-torsors $\mathscr{L}_{1}, \mathscr{L}_{2}$ and any $F$ in $\operatorname{Hom}_{\mathscr{P}}\left(\mathscr{L}_{1}, \mathscr{L}_{2}\right)$, a quasi-inverse $F^{-1}$ of $F$ together with an isomorphism $F^{-1} F \simeq$ id.

Moreover, $B \mathscr{P}$ is a category enriched over itself. That is, for any $\mathscr{P}$-torsors $\mathscr{L}_{1}, \mathscr{L}_{2}$ the category $\operatorname{Hom}_{\mathscr{P}}\left(\mathscr{L}_{1}, \mathscr{L}_{2}\right)$ is again a $\mathscr{P}$-torsor, where an action of $\mathscr{P}$ on $\operatorname{Hom}_{\mathscr{P}}\left(\mathscr{L}_{1}, \mathscr{L}_{2}\right)$ is defined as follows: for any $z \in \mathscr{P}, v \in \mathscr{L}_{1}, F \in \operatorname{Hom}_{\mathscr{P}}\left(\mathscr{L}_{1}, \mathscr{L}_{2}\right)$ we put $z+F \in \operatorname{Hom}_{\mathscr{P}}\left(\mathscr{L}_{1}, \mathscr{L}_{2}\right)$ as $(z+F)(v):=z+F(v)$. Now the isomorphism $\lambda$ for the equivalence $z+F$ is defined by means of the braiding maps $c$ in $\mathscr{P}$ (commutativity constraints from Section 2A). Then the diagram (2-7) for the equivalence $z+F$ follows from hexagon diagram (2-2). It is clear that this definition is extended to the definition of a bifunctor

$$
+: \mathscr{P} \times \operatorname{Hom}_{\mathscr{P}}\left(\mathscr{L}_{1}, \mathscr{L}_{2}\right) \rightarrow \operatorname{Hom}_{\mathscr{P}}\left(\mathscr{L}_{1}, \mathscr{L}_{2}\right)
$$

such that the axioms of $\mathscr{P}$-torsor are satisfied (see the beginning of this section).

We note that to prove that the category $B \mathscr{P}$ is enriched over itself we used the commutativity constraints in $\mathscr{P}$. The commutativity constraints will be important also below to define the sum of two $\mathscr{P}$-torsors.

The category $B \mathscr{P}$ furthermore forms a Picard 2-groupoid. We will not make the definition of Picard 2-groupoids precise. (However, one refers to [Kapranov and Voevodsky 1994; Breen 1994] for details). We will only describe the Picard structure on $B \mathscr{P}$ in the way we need.

First, if $\mathscr{L}_{1}, \mathscr{L}_{2}$ are two $\mathscr{P}$-torsors, then $\mathscr{L}_{1}+\mathscr{L}_{2}$ is defined to be the category whose objects are pairs $(v, w)$, where $v \in \mathscr{L}_{1}$ and $w \in \mathscr{L}_{2}$. The morphisms from $(v, w)$ to $\left(v^{\prime}, w^{\prime}\right)$ are defined as the equivalence classes of triples $\left(x, \varphi_{1}, \varphi_{2}\right)$, where $x \in \mathscr{P}, \varphi_{1} \in \operatorname{Hom}_{\mathscr{L}_{1}}\left(v, x+v^{\prime}\right)$ and $\varphi_{2} \in \operatorname{Hom}_{\mathscr{L}_{2}}\left(x+w, w^{\prime}\right)$, and $\left(x, \varphi_{1}, \varphi_{2}\right) \sim$ $\left(y, \phi_{1}, \phi_{2}\right)$ if there exists a map $f: x \rightarrow y$ such that $\phi_{1}=f\left(\varphi_{1}\right)$ and $\varphi_{2}=f\left(\phi_{2}\right)$. The identity in $\operatorname{Hom}_{\mathscr{L}_{1}+\mathscr{L}_{2}}((v, w),(v, w))$ and the composition

$$
\begin{aligned}
\operatorname{Hom}_{\mathscr{L}_{1}+\mathscr{L}_{2}}\left((v, w),\left(v^{\prime}, w^{\prime}\right)\right) \times \operatorname{Hom}_{\mathscr{L}_{1}+\mathscr{L}_{2}}\left(\left(v^{\prime}, w^{\prime}\right)\right. & \left.,\left(v^{\prime \prime}, w^{\prime \prime}\right)\right) \\
& \rightarrow \operatorname{Hom}_{\mathscr{L}_{1}+\mathscr{L}_{2}}\left((v, w),\left(v^{\prime \prime}, w^{\prime \prime}\right)\right)
\end{aligned}
$$

are clear. (To define the composition we have to use the commutativity constraints in $B \mathscr{P}$.) So $\mathscr{L}_{1}+\mathscr{L}_{2}$ is a category. Define the action of $\mathscr{P}$ on $\mathscr{L}_{1}+\mathscr{L}_{2}$ by 


$$
x+(v, w):=(x+v, w) .
$$

The natural isomorphism $(x+y)+(v, w) \simeq x+(y+(v, w))$ is the obvious one. It is easy to check that $\mathscr{L}_{1}+\mathscr{L}_{2}$ is a $\mathscr{P}$-torsor.

There is an obvious 1 -isomorphism of $\mathscr{P}$-torsors

$$
A:\left(\mathscr{L}_{1}+\mathscr{L}_{2}\right)+\mathscr{L}_{3} \simeq \mathscr{L}_{1}+\left(\mathscr{L}_{2}+\mathscr{L}_{3}\right)
$$

which is the associativity constraint. Namely, objects in $\left(\mathscr{L}_{1}+\mathscr{L}_{2}\right)+\mathscr{L}_{3}$ and in $\mathscr{L}_{1}+\left(\mathscr{L}_{2}+\mathscr{L}_{3}\right)$ are both canonically bijective to triples $\left(v_{1}, v_{2}, v_{3}\right)$ where $v_{i} \in \mathscr{L}_{i}$. Then $A$ is identity on objects. A morphism from $\left(v_{1}, v_{2}, v_{3}\right)$ to $\left(w_{1}, w_{2}, w_{3}\right)$ in $\left(\mathscr{L}_{1}+\mathscr{L}_{2}\right)+\mathscr{L}_{3}$ is of the form $\left(x,\left(y, \varphi_{1}, \varphi_{2}\right), \varphi_{3}\right)$, where $x, y \in \mathscr{P}, \varphi_{1}: v_{1} \rightarrow$ $y+\left(x+w_{1}\right), \varphi_{2}: y+v_{2} \rightarrow w_{2}, \varphi_{3}: x+v_{3} \rightarrow w_{3}$. Then $A$ maps $\left(x,\left(y, \varphi_{1}, \varphi_{2}\right), \varphi_{3}\right)$ to $\left(x+y, \varphi_{1}^{\prime},\left(x, \varphi_{2}^{\prime}, \varphi_{3}^{\prime}\right)\right)$, where $\varphi_{1}^{\prime}: v_{1} \rightarrow(x+y)+w_{1}$ comes from

$$
v_{1} \stackrel{\varphi_{1}}{\rightarrow} y+\left(x+w_{1}\right) \simeq(y+x)+w_{1} \simeq(x+y)+w_{1},
$$

$\varphi_{2}^{\prime}:(x+y)+v_{2} \rightarrow x+w_{2}$ comes from

$$
(x+y)+v_{2} \simeq x+\left(y+v_{2}\right) \stackrel{x+\varphi_{2}}{\longrightarrow} x+w_{2},
$$

and $\varphi_{3}^{\prime}: x+v_{3} \rightarrow w_{3}$ is the same as $\varphi_{3}$.

To complete the definition of $A$, we should specify for every $x \in \mathscr{P},\left(v_{1}, v_{2}, v_{3}\right) \in$ $\left(\mathscr{L}_{1}+\mathscr{L}_{2}\right)+\mathscr{L}_{3}$, an isomorphism $\lambda: A\left(x+\left(v_{1}, v_{2}, v_{3}\right)\right) \simeq x+A\left(v_{1}, v_{2}, v_{3}\right)$ such that the diagram (2-7) is commutative for $F=A$. It is clear that $\lambda=\mathrm{id}:\left(x+v_{1}, v_{2}, v_{3}\right)=$ $\left(x+v_{1}, v_{2}, v_{3}\right)$ will suffice for this purpose.

It is clear from definition of $A$ that we can similarly construct a 1-morphism $A^{-1}$ of $\mathscr{P}$-torsors such that the following equalities are satisfied:

$$
A^{-1} A=A A^{-1}=\mathrm{id} \text {. }
$$

From above construction of the associativity constraints (1-morphisms $A$ and $A^{-1}$ ) it follows that for any $\mathscr{P}$-torsors $\mathscr{L}_{1}, \mathscr{L}_{2}, \mathscr{L}_{3}, \mathscr{L}_{4}$ the following diagram of 1-morphisms (pentagon diagram) is commutative:

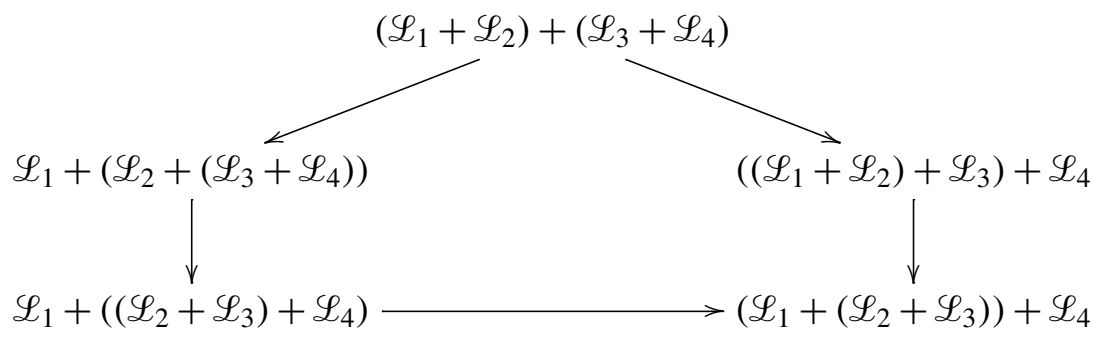

(To prove this diagram we note that this diagram is evident for objects from category $\left(\mathscr{L}_{1}+\mathscr{L}_{2}\right)+\left(\mathscr{L}_{3}+\mathscr{L}_{4}\right)$. To verify this diagram for morphisms from this 
category one needs to make some routine calculations. The analogous reasonings are also applied to the diagram (2-13) below.)

The following axioms are satisfied in the category $B \mathscr{P}$ and describe the functoriality of the associativity constraints. Let $\mathscr{L}_{1}, \mathscr{L}_{2}, \mathscr{L}_{3}, \mathscr{L}_{1}^{\prime}$ be any $\mathscr{P}$-torsors, and $\mathscr{L}_{1} \rightarrow \mathscr{L}_{1}^{\prime}$ be any 1-morphism of $\mathscr{P}$-torsors, then the following diagram of 1-morphisms is commutative:

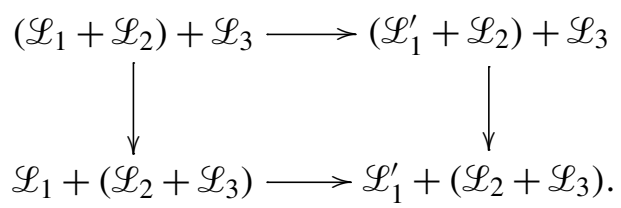

Let $\mathscr{L}_{1}, \mathscr{L}_{2}, \mathscr{L}_{3}, \mathscr{L}_{2}^{\prime}$ be any $\mathscr{P}$-torsors, and $\mathscr{L}_{2} \rightarrow \mathscr{L}_{2}^{\prime}$ be any 1 -morphism of $\mathscr{P}$ torsors, then the following diagram of 1-morphisms is commutative:

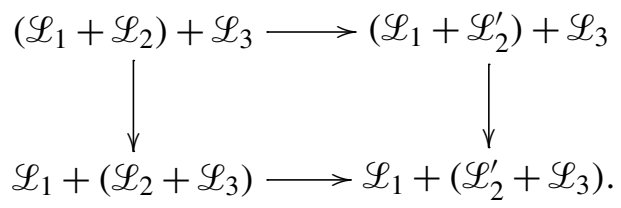

Let $\mathscr{L}_{1}, \mathscr{L}_{2}, \mathscr{L}_{3}, \mathscr{L}_{3}^{\prime}$ be any $\mathscr{P}$-torsors, and $\mathscr{L}_{3} \rightarrow \mathscr{L}_{3}^{\prime}$ be any 1 -morphism of $\mathscr{P}_{-}$ torsors, then the following diagram of 1-morphisms is commutative:

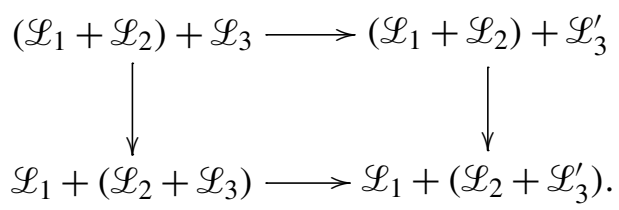

(In diagrams (2-10)-(2-12) the vertical arrows are the associativity constraints.)

Next we define the commutativity constraints. Recall that we have chosen for each $x \in \mathscr{P}$ its inverse $\left(-x, \phi_{x}\right)$, and then obtained the isomorphism (2-4). This gives an obvious 1-isomorphism

$$
C: \mathscr{L}_{1}+\mathscr{L}_{2} \simeq \mathscr{L}_{2}+\mathscr{L}_{1}
$$

Namely, $C$ will map the object $\left(v_{1}, v_{2}\right)$ to $\left(v_{2}, v_{1}\right)$, and $\left(x, \varphi_{1}, \varphi_{2}\right):\left(v_{1}, v_{2}\right) \rightarrow$ $\left(w_{1}, w_{2}\right)$ to $\left(-x, \varphi_{1}^{\prime}, \varphi_{2}^{\prime}\right):\left(v_{2}, v_{1}\right) \rightarrow\left(w_{2}, w_{1}\right)$, where

$$
\begin{aligned}
& \varphi_{1}^{\prime}: v_{2} \simeq e+v_{2} \simeq(-x+x)+v_{2} \simeq-x+\left(x+v_{2}\right) \stackrel{-x+\varphi_{2}}{\rightarrow}-x+w_{2}, \\
& \varphi_{2}^{\prime}:-x+v_{1} \stackrel{-x+\varphi_{1}}{\rightarrow}-x+\left(x+w_{1}\right) \simeq(-x+x)+w_{1} \simeq e+w_{1} \simeq w_{1} .
\end{aligned}
$$

We also define for each $x \in \mathscr{P},\left(v_{1}, v_{2}\right) \in \mathscr{L}_{1}+\mathscr{L}_{2}$, the isomorphism

$$
\lambda: C\left(x+\left(v_{1}, v_{2}\right)\right)=\left(v_{2}, x+v_{1}\right) \rightarrow x+C\left(v_{1}, v_{2}\right)=\left(x+v_{2}, v_{1}\right)
$$


by $\lambda=\left(-x, \varphi_{1}, \varphi_{2}\right)$, where $\varphi_{1}: v_{2} \simeq(-x+x)+v_{2} \simeq-x+\left(x+v_{2}\right)$ and $\varphi_{2}$ : $-x+\left(x+v_{1}\right) \simeq(-x+x)+v_{1} \simeq v_{1}$.

In addition, by (2-3), there is an equality of 1-morphisms $C^{2}=\mathrm{id}$.

The commutativity constrains together with the associativity constrains satisfy the hexagon diagram; i.e., for any $\mathscr{P}$-torsors $\mathscr{L}_{1}, \mathscr{L}_{2}, \mathscr{L}_{3}$ the following diagram of 1-morphisms is commutative:

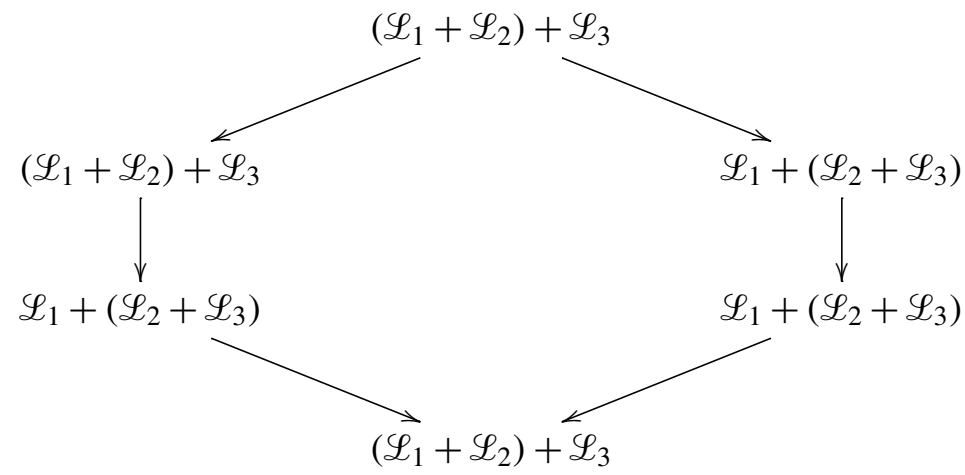

The following axiom is satisfied in the category $B \mathscr{P}$ and describes the functoriality of the commutativity constraints. Let $\mathscr{L}_{1}, \mathscr{L}_{2}, \mathscr{L}_{1}^{\prime}$ be any $\mathscr{P}$-torsors, and $\mathscr{L}_{1} \rightarrow \mathscr{L}_{1}^{\prime}$ be any 1-morphism of $\mathscr{P}$-torsors, then the following diagram of 1 morphisms is commutative:

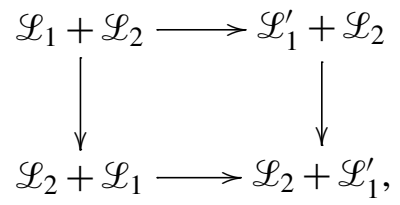

where the vertical arrows are the commutativity constraints.

By regarding $\mathscr{P}$ as a $\mathscr{P}$-torsor, there is a canonical 1-isomorphism of $\mathscr{P}$-torsors $\mathscr{P}+\mathscr{L} \rightarrow \mathscr{L},(x, v) \mapsto x+v$ satisfying the associativity and commutativity constraints. This means that $\mathscr{P}$ is the unit in $B \mathscr{P}$. For each $\mathscr{L} \in B \mathscr{P}$, we have an object

$$
-\mathscr{L}:=\operatorname{Hom}_{\mathscr{P}}(\mathscr{L}, \mathscr{P})
$$

together with a natural 1 -isomorphism of $\mathscr{P}$-torsors $\varphi_{\mathscr{L}}: \mathscr{L}+(-\mathscr{L}) \simeq \mathscr{P}$. This object is called an inverse of $\mathscr{L}$.

For $\mathscr{L}$ a $\mathscr{P}$-torsor, $\operatorname{Hom}_{\mathscr{P}}(\mathscr{L}, \mathscr{L})$ is a natural monoidal groupoid (by composition). The natural homomorphism

$$
\mathscr{L}: \mathscr{P} \rightarrow \operatorname{Hom}_{\mathscr{P}}(\mathscr{L}, \mathscr{L})
$$


given by $\mathscr{L}(z)=z+\mathrm{id}^{5}$ is a 1 -isomorphism of monoidal groupoids. We will fix once and for all its inverse, i.e., we choose an 1-isomorphism of monoidal groupoids

$$
\mathscr{L}^{-1}: \operatorname{Hom}_{\mathscr{P}}(\mathscr{L}, \mathscr{L}) \rightarrow \mathscr{P}
$$

together with a 2-isomorphism $\mathscr{L}^{-1} \circ \mathscr{L} \simeq$ id.

Remark 2.3. We constructed some "semistrict" version of Picard 2-groupoid, because diagrams (2-9)-(2-14) are true in $B \mathscr{P}$ for 1-morphisms without consideration of additional 2-morphisms which involve higher coherence axioms for braided monoidal 2-categories as in [Kapranov and Voevodsky 1994] and [Baez and Neuchl 1996]. Besides, from the equality $C^{2}=$ id we obtain at one stroke that our 2category $B \mathscr{P}$ is strongly braided, i.e, the diagram (8.4.6) in [Breen 1994, p. 149] holds. Let us mention that in loc. cit., the commutativity constraint $C$ is denoted by $R$.

2D. The case $H^{\mathbf{1}}(B G, \mathscr{P})$. Let $\mathscr{P}$ be a Picard groupoid, and $G$ be a group. Then we define $H^{1}(B G, \mathscr{P})$ to be the Picard groupoid of homomorphisms from $G$ to $\mathscr{P}$. That is, the objects are monoidal functors from $G$ to $\mathscr{P}$, where $G$ is regarded as a discrete monoidal category (the monoidal groupoid whose objects are elements of $G$ and whose only morphisms are the unit morphisms of objects), and morphisms between these monoidal functors are monoidal natural transformations. In concrete terms, $f \in H^{1}(B G, \mathscr{P})$ is a functor $f: G \rightarrow \mathscr{P}$, together with isomorphisms

$$
f\left(g g^{\prime}\right) \simeq f(g)+f\left(g^{\prime}\right)
$$

which are compatible with the associativity constraints. The monoidal structure on $H^{1}(B G, \mathscr{P})$ is given by $\left(f+f^{\prime}\right)(g)=f(g)+f\left(g^{\prime}\right)$. The natural isomorphism $\left(f+f^{\prime}\right)\left(g g^{\prime}\right) \simeq\left(f+f^{\prime}\right)(g)+\left(f+f^{\prime}\right)\left(g^{\prime}\right)$ is the obvious one. The associativity constraints and the commutativity constraints on $H^{1}(B G, \mathscr{P})$ are clear. Let $(e, \varphi)$ be a unit of $\mathscr{P}$, and $e$ is regarded as a discrete Picard groupoid with one object. Then $f: G \rightarrow \mathscr{P}$ is called trivial if it is isomorphic to $G \rightarrow e \rightarrow \mathscr{P}$.

Example 2.4. If $\mathscr{P}=B A$, then $H^{1}(B G, B A)$ is equivalent to the category of central extensions of $G$ by $A$ as Picard groupoids.

Let $Z_{2} \subset G \times G$ be the subset of commuting elements, so that if $G$ itself is an abelian group, then $Z_{2}=G \times G$. In general, fix $g \in G$, then $Z_{2} \cap(G \times g) \simeq$ $Z_{2} \cap(g \times G) \simeq Z_{G}(g)$, the centralizer of $g$ in $G$.

Lemma-Definition 2.5. There is a well defined antisymmetric bimultiplicative map $\operatorname{Comm}(f): Z_{2} \rightarrow \pi_{1}(\mathscr{P})=\operatorname{End}_{\mathscr{P}}(e)$.

\footnotetext{
${ }^{5}$ Recall that we constructed the bifunctor $+: \mathscr{P} \times \operatorname{Hom}_{\mathscr{P}}(\mathscr{L}, \mathscr{L}) \rightarrow \operatorname{Hom}_{\mathscr{P}}(\mathscr{L}, \mathscr{L})$ in $(2-8)$.
} 
Proof. The definition of $\operatorname{Comm}(f)$ is as follows. For $g_{1}, g_{2} \in Z_{2}$, we have

$$
f\left(g_{1} g_{2}\right) \simeq f\left(g_{1}\right)+f\left(g_{2}\right) \simeq f\left(g_{2}\right)+f\left(g_{1}\right) \simeq f\left(g_{2} g_{1}\right)=f\left(g_{1} g_{2}\right),
$$

where the first and the third isomorphisms come from the constraints for the homomorphism $f$, and the second isomorphism comes from the commutativity constraints of the Picard groupoid $\mathscr{P}$. We thus obtain an element

$$
\operatorname{Comm}(f)\left(g_{1}, g_{2}\right) \in \operatorname{Aut} \mathscr{P}\left(f\left(g_{1} g_{2}\right)\right) \simeq \pi_{1}(\mathscr{P}) .
$$

Since $\mathscr{P}$ is Picard, i.e., the commutativity constraints satisfy

$$
c_{f\left(g_{1}\right), f\left(g_{2}\right)}=c_{f\left(g_{2}\right), f\left(g_{1}\right)}^{-1},
$$

the map Comm is antisymmetric. One checks directly by diagram that Comm $(f)$ is also bimultiplicative (see the analogous diagram (2-29) below).

Here we will give another proof of bimultiplicativity whose higher categorical analog we will use in the proof of Lemma-Definition 2.13. We construct the following category $H_{f}$, where objects of $H_{f}$ are all possible expressions $\left.f\left(g_{1}\right)+\cdots+f\left(g_{k}\right):=\left(\cdots\left(f\left(g_{1}\right)+f\left(g_{2}\right)\right)+f\left(g_{3}\right)\right)+\cdots\right)+f\left(g_{k}\right)$, where $g_{i} \in G$, and morphisms in $H_{f}$ are defined as follows:

$$
\begin{aligned}
& \operatorname{Hom}_{H_{f}}\left(f\left(g_{i_{1}}\right)+\cdots+f\left(g_{i_{k}}\right), f\left(g_{j_{1}}\right)+\cdots+f\left(g_{j_{l}}\right)\right) \\
& \varnothing \begin{array}{cl}
\varnothing & \text { if } g_{i_{1}} \ldots g_{i_{k}} \neq g_{j_{1}} \ldots g_{j_{l}}, \\
\operatorname{Hom}_{\mathscr{P}}\left(f\left(g_{i_{1}}\right)+\cdots+f\left(g_{i_{k}}\right), f\left(g_{j_{1}}\right)+\cdots+f\left(g_{j_{l}}\right)\right) & \text { if } g_{i_{1}} \ldots g_{i_{k}}=g_{j_{1}} \ldots g_{j_{l}} .
\end{array}
\end{aligned}
$$

The category $H_{f}$ is a monoidal group-like groupoid (or $g r$-category), where the monoidal structure on $H_{f}$ is given in an obvious way by using the associativity constraints in the category $\mathscr{P}$. We have $\pi_{0}\left(H_{f}\right)=G$, and $H_{f}$ is equivalent to the trivial $g r$-category. We consider $\pi_{1}(\mathscr{P})$-torsor $E$ over $Z_{2}$ which is the commutator of $H_{f}$ (see [Breen 1999, §3]). The fiber of $E$ over $\left(g_{1}, g_{2}\right) \in Z_{2}$ is the set

$$
E_{g_{1}, g_{2}}=\operatorname{Hom}_{H_{f}}\left(f\left(g_{1}\right)+f\left(g_{2}\right), f\left(g_{2}\right)+f\left(g_{1}\right)\right) .
$$

The $\pi_{1}(\mathscr{P})$-torsor $E$ has a natural structure of a weak biextension of $Z_{2}$ by $\pi_{1}(\mathscr{P})$ (see [Breen 1999, Proposition 3.1]), i.e., there are partial composition laws on $E$ which are compatible (see also (2-22)). Now the commutativity constraints $c_{f\left(g_{1}\right), f\left(g_{2}\right)}$ give a section of $E$ over $Z_{2}$ which is compatible with partial composition laws on $E$, i.e., "bimultiplicative". (The compatibility of this section with the composition laws follows at once from the definition of the partial composition laws on $E$ and the hexagon diagram (2-2).) The other section of $E$ which is compatible with partial composition laws on $E$ is obtained as the composition of following two morphisms from definition of $f: f\left(g_{1}\right)+f\left(g_{2}\right) \simeq f\left(g_{1} g_{2}\right)=f\left(g_{2} g_{1}\right) \simeq f\left(g_{2}\right)+f\left(g_{1}\right)$. (The compatibility of this section with composition laws follows from diagrams 
(3.10) and (1.4) of [Breen 1999], because of the compatibility of our homomorphism $f$ with the associativity constraints.) Now the difference between the first section and the second section coincides with $\operatorname{Comm}(f)$, which is, thus, a bimultiplicative function, because both sections are "bimultiplicative".

Remark 2.6. In [Breen 1999, §2] the notion of a weak biextension was intoduced only for $Z_{2}=B \times B$ where $B$ is an abelian group. Here, we generalize this notion by allowing $B$ to be non-commutative and by replacing $B \times B$ by $Z_{2}$. But all the axioms for partial composition laws in loc. cit. are still applicable in this setting. The same remark applies when we talk about $(2,2)$-extensions on page 308 .

Remark 2.7. It is clear that if $f \simeq f^{\prime}$ in $H^{1}(B G, \mathscr{P})$, then $\operatorname{Comm}(f)=\operatorname{Comm}\left(f^{\prime}\right)$.

Remark 2.8. When $\mathscr{P}=B A$, this construction reduces to the usual construction of inverse to the commutator pairing maps for central extensions.

Corollary 2.9. One has $\operatorname{Comm}\left(f+f^{\prime}\right)=\operatorname{Comm}(f)+\operatorname{Comm}\left(f^{\prime}\right)$.

Proof. It can be easily checked directly by diagrams. See, for example, analogous formulas and diagrams in (2-30)-(2-32) below.

Corollary 2.10. Assume that $G$ is abelian so that $Z_{2}=G \times G$. Then $\operatorname{Comm}(f)$ is trivial if and only if the 1-homomorphism $f$ is a 1-homomorphism of Picard groupoids. In particular, if the homomorphism $f$ is trivial, then $\operatorname{Comm}(f)$ is trivial.

Proof. This follows from diagram (2-5).

Together, these two corollaries can be rephrased as saying that if $G$ is abelian, then there is an exact sequence of Picard groupoids

$$
1 \rightarrow \operatorname{Hom}(G, \mathscr{P}) \rightarrow H^{1}(B G, \mathscr{P}) \rightarrow \operatorname{Hom}\left(\bigwedge^{2} G, \pi_{1}(\mathscr{P})\right) .
$$

2E. The case $\boldsymbol{H}^{2}(B G, \mathscr{P})$. If $\mathscr{P}^{\prime}$ is a Picard $n$-groupoid, and $G$ is a group, one should be able to define $H^{1}\left(B G, \mathscr{P}^{\prime}\right)$ as the Picard $n$-groupoid of homomorphisms from $G$ to $\mathscr{P}^{\prime}$. When $n=1$, this is what we discussed in the previous subsection. The next step for consideration is $n=2$. Again, instead of discussing general Picard 2-groupoids, we will focus on the case when $\mathscr{P}^{\prime}=B \mathscr{P}$, where $\mathscr{P}$ is a Picard groupoid. Then one can interpret $H^{1}(B G, B \mathscr{P})$ as the Picard groupoid ${ }^{6}$ of central extensions of the group $G$ by the Picard groupoid $\mathscr{P}$. For this reason, we also denote $H^{1}(B G, B \mathscr{P})$ by $H^{2}(B G, \mathscr{P})$.

In concrete terms, an object $\mathscr{L}$ in $H^{2}(B G, \mathscr{P})$ is a rule to assign to every $g \in G$ a $\mathscr{P}_{\text {-torsor }} \mathscr{L}_{g}$, and to every $g, g^{\prime}$ an equivalence $\mathscr{L}_{g g^{\prime}} \simeq \mathscr{L}_{g}+\mathscr{L}_{g^{\prime}}$ of $\mathscr{P}_{\text {-torsors, and }}$

${ }^{6}$ As we just mentioned, it is in fact a Picard 2-groupoid. 
to every $g, g^{\prime}, g^{\prime \prime}$ an isomorphism between two equivalences

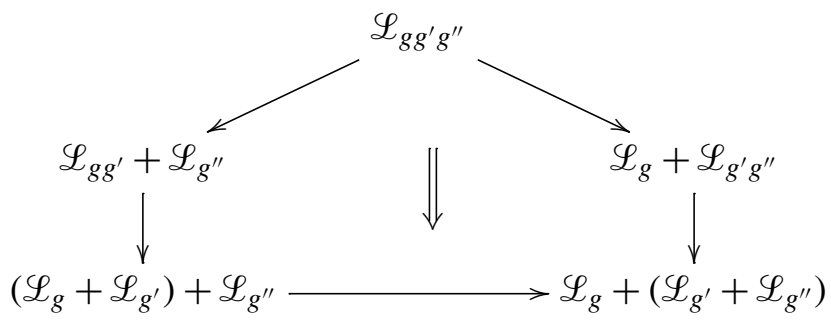

such that for every $g, g^{\prime}, g^{\prime \prime}, g^{\prime \prime \prime}$, the natural compatibility condition holds, which we describe below.

Remark 2.11. Our notation for the 2 -arrow in diagram (2-17) is symbolic, and is distinct from the traditional notation of 2-arrows in a 2-category, because this 2-arrow is between a pair of 1 -arrows from $\mathscr{L}_{g g^{\prime} g^{\prime \prime}}$ to $\mathscr{L}_{g}+\left(\mathscr{L}_{g}^{\prime}+\mathscr{L}_{g}^{\prime \prime}\right)$ and should be written horizontally from left to right rather than vertically. This notation for the 2-arrow will be important for us in diagram (2-28).

We define an isomorphism between two central extensions of $G$ by $\mathscr{P}$. An isomorphism between two central extensions $\mathscr{L}, \mathscr{L}^{\prime}$ is a rule which assigns to any $g$ a $\mathscr{P}$-torsor 1-isomorphism $\mathscr{L}_{g} \simeq \mathscr{L}_{g}^{\prime}$, and to any $g, g^{\prime}$ the following 2-isomorphism

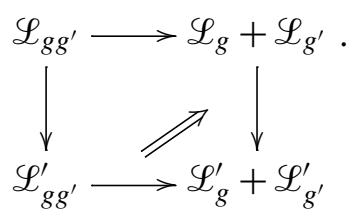

In addition, these assignments have to be compatible with diagram (2-17) in an obvious way.

Now we describe the compatibility condition which we need after diagram (2-17). If we don't consider the associativity constraints in category $B \mathscr{P}$, then the 2-arrows induced by the one in (2-17) should satisfy the compatibility condition described by the following cube:

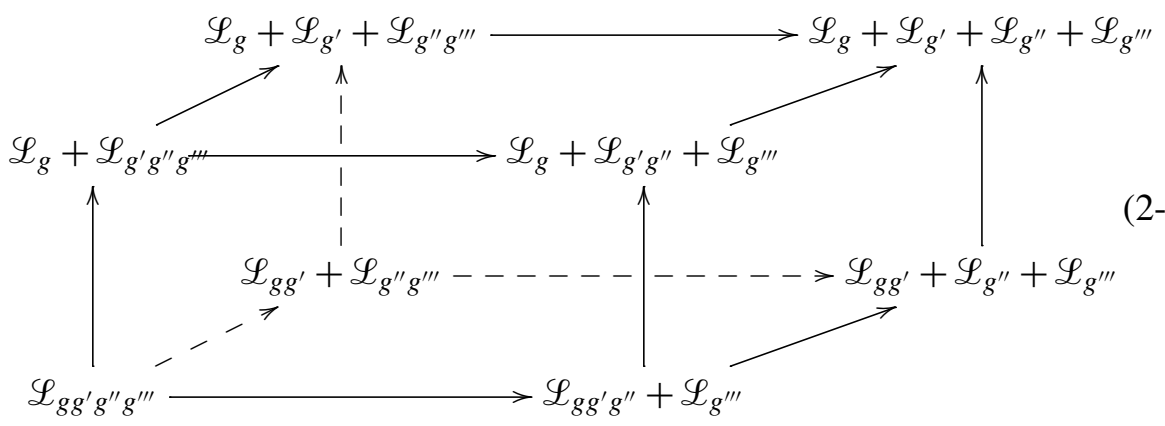


To obtain the correct compatibility diagram for 2-morphisms, we have to replace in diagram (2-18) the arrow (an edge of cube)

$$
\mathscr{L}_{g g^{\prime}}+\mathscr{L}_{g^{\prime \prime}}+\mathscr{L}_{g^{\prime \prime \prime}} \longrightarrow \mathscr{L}_{g}+\mathscr{L}_{g^{\prime}}+\mathscr{L}_{g^{\prime \prime}}+\mathscr{L}_{g^{\prime \prime \prime}}
$$

by the following commutative diagram of 1-morphisms in the category $B \mathscr{P}$

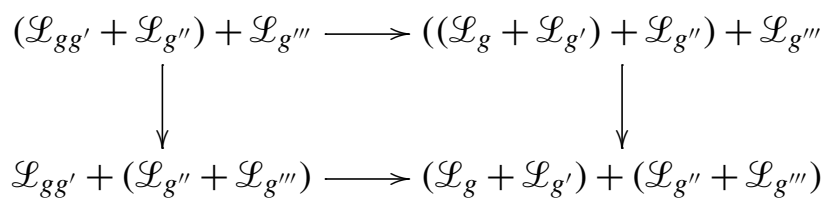

(where the vertical arrows are associativity constraints); we have to replace in diagram (2-18) the arrow (an edge of the cube)

$$
\mathscr{L}_{g}+\mathscr{L}_{g^{\prime} g^{\prime \prime}}+\mathscr{L}_{g^{\prime \prime \prime}} \longrightarrow \mathscr{L}_{g}+\mathscr{L}_{g^{\prime}}+\mathscr{L}_{g^{\prime \prime}}+\mathscr{L}_{g^{\prime \prime \prime}}
$$

by the following commutative diagram of 1-morphisms in the category $B \mathscr{P}$ :

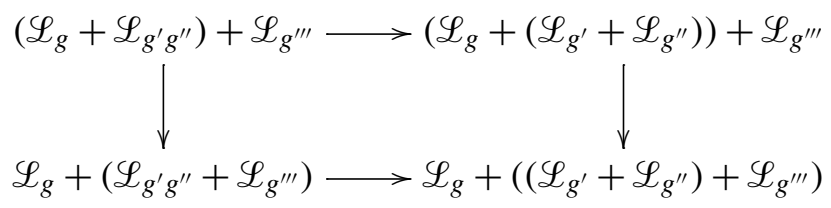

(where vertical arrows are associativity constraints); we have to replace in diagram (2-18) the arrow (an edge of the cube)

$$
\mathscr{L}_{g}+\mathscr{L}_{g^{\prime}}+\mathscr{L}_{g^{\prime \prime} g^{\prime \prime \prime}} \longrightarrow \mathscr{L}_{g}+\mathscr{L}_{g^{\prime}}+\mathscr{L}_{g^{\prime \prime}}+\mathscr{L}_{g^{\prime \prime \prime}}
$$

by the following commutative diagram of 1-morphisms in the category $B \mathscr{P}$ :

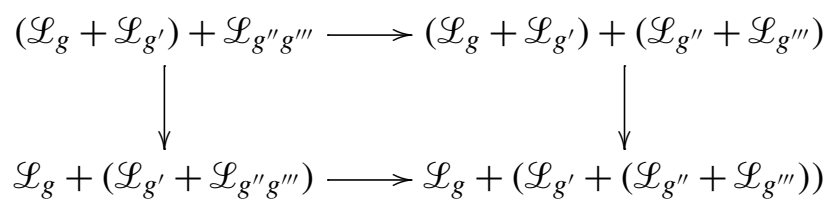

(where vertical arrows are associativity constraints). Besides, instead of the vertex $\mathscr{L}_{g}+\mathscr{L}_{g^{\prime}}+\mathscr{L}_{g^{\prime \prime}}+\mathscr{L}_{g^{\prime \prime \prime}}$ in diagram (2-18) we insert the commutative diagram which is the modification of pentagon diagram (2-9) for $\mathscr{L}_{g}, \mathscr{L}_{g^{\prime}}, \mathscr{L}_{g^{\prime \prime}}, \mathscr{L}_{g^{\prime \prime \prime}}$, and this diagram is always true in category $B \mathscr{P}$. The correct compatibility diagram for 2-morphisms from diagrams (2-17) has 15 vertices.

We note that diagrams (2-19)-(2-21) are commutative for 1-morphisms; that is, the corresponding 2-isomorphisms equal identity morphisms. These diagrams express the "functoriality" of associativity constraints in $B \mathscr{P}$ and follow from axiomsdiagrams (2-10)-(2-12) in category $B \mathscr{P}$. 
The trivial central extension of $G$ by $\mathscr{P}$, which we will denote by the same letter $\mathscr{P}$, is the rule that assigns to every $g \in G$ the trivial $\mathscr{P}$-torsor $\mathscr{P}$, to every $g, g^{\prime}$ the natural 1-isomorphism $\mathscr{P} \simeq \mathscr{P}+\mathscr{P},{ }^{7}$ and to every $g, g^{\prime}, g^{\prime \prime}$ the corresponding natural 2-isomorphism.

Remark 2.12. A central extension $\mathscr{L}$ of $G$ by $\mathscr{P}$ gives rise to a gr-category, $\tilde{\mathscr{L}}$, together with a short exact sequence of gr-categories in the sense of [Breen 1992, Definition 2.1.2]

$$
1 \rightarrow \mathscr{P} \stackrel{i}{\rightarrow} \tilde{\mathscr{L}} \stackrel{\pi}{\rightarrow} G \rightarrow 1 .
$$

Namely, as a category, $\tilde{\mathscr{L}}=\bigcup_{g \in G} \mathscr{L}_{g}$. Then the natural equivalence $\mathscr{L}_{g g^{\prime}} \simeq \mathscr{L}_{g}+\mathscr{L}_{g^{\prime}}$ together with the compatibility conditions endows $\tilde{\mathscr{L}}$ with a gr-category structure. The natural morphism $\pi: \tilde{\mathscr{L}} \rightarrow G$ is clearly monoidal, and one can show that ker $\pi=\mathscr{L}_{e}$ is 1 -isomorphic to $\mathscr{P}$.

As is shown in loc. cit., such a short exact sequence endows every $\tilde{\mathscr{L}}_{g}:=$ $\pi^{-1}(g)=\mathscr{L}_{g}$ with a $\mathscr{P}$-bitorsor structure. This $\mathscr{P}$-bitorsor structure is nothing but the canonical $\mathscr{P}$-bitorsor structure on $\mathscr{L}_{g}$ (observe that the morphism $\mathscr{E}: \mathscr{P} \rightarrow$ $\operatorname{Hom}_{\mathscr{P}}\left(\mathscr{L}_{g}, \mathscr{L}_{g}\right)$ as in (2-15) induces a canonical $\mathscr{P}$-bitorsor structure on $\left.\mathscr{L}_{g}\right)$.

The upshot is that an object $\mathscr{L}$ in $H^{2}(B G, \mathscr{P})$ gives rise to a categorical generalization of a central extension of a group by an abelian group. This justifies our terminology. Indeed, one can define a central extension of $G$ by $\mathscr{P}$ as a short exact sequence as above such that the induced $\mathscr{P}$-bitorsor structure on each $\tilde{\mathscr{L}}_{g}$ is the canonical one induced from its left $\mathscr{P}$-torsor structure. Since we do not use this second definition, we will not make it precise.

Finally, let us define the Picard structure on $H^{2}(B G, \mathscr{P})$. Let $\mathscr{L}$ and $\mathscr{L}^{\prime}$ be two central extensions of $G$ by $\mathscr{P}$. Then we define the central extension $\mathscr{L}+\mathscr{L}^{\prime}$ by the following way:

$$
\left(\mathscr{L}+\mathscr{L}^{\prime}\right)_{g}:=\mathscr{L}_{g}+\mathscr{L}_{g}^{\prime},
$$

and the equivalence $\left(\mathscr{L}+\mathscr{L}^{\prime}\right)_{g g^{\prime}} \simeq\left(\mathscr{L}+\mathscr{L}^{\prime}\right)_{g}+\left(\mathscr{L}+\mathscr{L}^{\prime}\right)_{g^{\prime}}$ as the composition of the equivalences

$$
\begin{aligned}
\left(\mathscr{L}+\mathscr{L}^{\prime}\right)_{g g^{\prime}}=\mathscr{L}_{g g^{\prime}}+\mathscr{L}_{g g^{\prime}}^{\prime} & \simeq\left(\mathscr{L}_{g}+\mathscr{L}_{g^{\prime}}\right)+\left(\mathscr{L}_{g}^{\prime}+\mathscr{L}_{g^{\prime}}^{\prime}\right) \\
& \simeq\left(\mathscr{L}_{g}+\mathscr{L}_{g}^{\prime}\right)+\left(\mathscr{L}_{g^{\prime}}+\mathscr{L}_{g^{\prime}}^{\prime}\right)=\left(\mathscr{L}+\mathscr{L}^{\prime}\right)_{g}+\left(\mathscr{L}+\mathscr{L}^{\prime}\right)_{g^{\prime}} .
\end{aligned}
$$

The corresponding 2-isomorphism for central extension $\mathscr{L}+\mathscr{L}^{\prime}$ and any elements $g, g^{\prime}, g^{\prime \prime}$ of $G$ follows from diagrams (2-17) for central extensions $\mathscr{L}$ and $\mathscr{L}^{\prime}$. The further compatibility conditions for these 2-isomorphisms hold as in diagrams (2-18)-(2-21), since they follow at once from the corresponding diagrams for central extensions $\mathscr{L}$ and $\mathscr{L}^{\prime}$.

\footnotetext{
${ }^{7}$ Naturality means this 1 -isomorphism is the chosen quasi-inverse of the natural 1-isomorphism $\mathscr{P}+\mathscr{P} \rightarrow \mathscr{P}$.
} 
Again, let $Z_{2}$ denote the subset of $G \times G$ consisting of commuting elements. We will give a categorical analog of Lemma-Definition 2.5. For this purpose, let us first explain some terminology. A 1-morphism $f: Z_{2} \rightarrow \mathscr{P}$ is called bimultiplicative if for fixed $g \in G,\left(Z_{G}(g), g\right) \subset Z_{2} \rightarrow \mathscr{P}$ and $\left(g, Z_{G}(g)\right) \subset Z_{2} \rightarrow \mathscr{P}$ are homomorphisms, i.e., monoidal functors from discrete monoidal categories $\left(Z_{G}(g), g\right)$ and $\left(g, Z_{G}(g)\right)$ to $\mathscr{P}$. In addition, the following diagram must be commutative (which is the compatibility condition between these two homomorphisms):

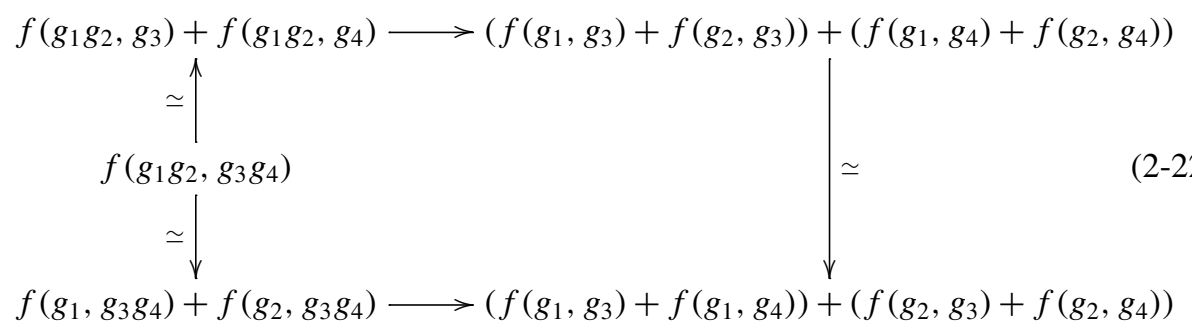

When $\mathscr{P}=B A$, a bimultiplicative 1-morphism from $Z_{2} \rightarrow B A$ is the same as a weak biextension of $Z_{2}$ by $A$ as defined in [Breen 1999, §2] (see also Remark 2.6).

A 1-morphism $f: Z_{2} \rightarrow \mathscr{P}$ is called antisymmetric if there is a 2-isomorphism $\theta: f \simeq-f \circ \sigma$, where $\sigma$ is the natural flip on $Z_{2}$, such that for any $\left(g_{1}, g_{2}\right) \in Z_{2}$, the following diagram is commutative:

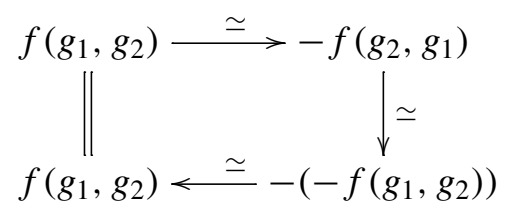

We need some more terminology. Following [Breen 1999, §7], we define a weak $(2,2)$-extension of $Z_{2}$ by $\mathscr{P}$ as a rule which assigns to every $\left(g, g^{\prime}\right) \in Z_{2}$ a $\mathscr{P}$-torsor $\mathscr{E}_{\left(g, g^{\prime}\right)}$ such that its restrictions to $\left(g, Z_{G}(g)\right)$ and $\left.Z_{G}(g), g\right)$ are central extensions of $Z_{G}(g)$ by $\mathscr{P}$, and that the corresponding diagram (2-22) is 2-commutative (i.e., commutative modulo some 2-isomorphism), and these 2-isomorphisms satisfy further compatibility conditions (see (7.1), (7.3) in loc. cit. where these compatibility conditions are carefully spelt out).

Lemma-Definition 2.13. There is an antisymmetric bimultiplicative homomorphism $C_{2}^{\mathscr{L}}: Z_{2} \rightarrow \mathscr{P}$.

Proof. As in the proof of 2.5, using the commutativity constraints $C: \mathscr{L}_{g}+\mathscr{L}_{g^{\prime}} \simeq$ $\mathscr{L}_{g^{\prime}}+\mathscr{L}_{g}$ in the category $B \mathscr{P}$, one constructs the following composition of 1 isomorphisms:

$$
\mathscr{L}_{g g^{\prime}} \simeq \mathscr{L}_{g}+\mathscr{L}_{g^{\prime}} \simeq \mathscr{L}_{g^{\prime}}+\mathscr{L}_{g} \simeq \mathscr{L}_{g^{\prime} g}=\mathscr{L}_{g g^{\prime}},
$$


for $\left(g, g^{\prime}\right) \in Z_{2}$. In this way, we obtain a functor $Z_{2} \rightarrow \operatorname{Hom}_{\mathscr{P}}\left(\mathscr{L}_{g g^{\prime}}, \mathscr{L}_{g g^{\prime}}\right)$. Using $\mathscr{L}^{-1}: \operatorname{Hom}_{\mathscr{P}}\left(\mathscr{L}_{g g^{\prime}}, \mathscr{L}_{g g^{\prime}}\right) \rightarrow \mathscr{P}($ see $(2-16))$, we get a morphism $C_{2}^{\mathscr{L}}: Z_{2} \rightarrow \mathscr{P}$.

We need to construct the following canonical isomorphisms $C_{2}^{\mathscr{L}}\left(g g^{\prime}, g^{\prime \prime}\right) \simeq C_{2}^{\mathscr{L}}\left(g, g^{\prime \prime}\right)+C_{2}^{\mathscr{L}}\left(g^{\prime}, g^{\prime \prime}\right), \quad C_{2}^{\mathscr{L}}\left(g, g^{\prime} g^{\prime \prime}\right) \simeq C_{2}^{\mathscr{L}}\left(g, g^{\prime}\right)+C_{2}^{\mathscr{L}}\left(g, g^{\prime \prime}\right)$,

satisfying the natural compatibility conditions. We now construct the first isomorphism. The second is similar. Let $\mathscr{L}: \mathscr{P} \rightarrow \operatorname{Hom}_{\mathscr{P}}\left(\mathscr{L}_{g g^{\prime} g^{\prime \prime}}, \mathscr{L}_{g g^{\prime} g^{\prime \prime}}\right)$ be the canonical equivalence as monoidal groupoids as in (2-15). It is enough to construct a canonical 2-isomorphism $\mathscr{L}\left(C_{2}^{\mathscr{L}}\left(g g^{\prime}, g^{\prime \prime}\right)\right) \simeq \mathscr{L}\left(C_{2}^{\mathscr{L}}\left(g, g^{\prime \prime}\right)+C_{2}^{\mathscr{L}}\left(g^{\prime}, g^{\prime \prime}\right)\right)$.

By the definition of the morphism $C_{2}^{\mathscr{L}}$, there is a canonical 2-isomorphism from 1-isomorphism $\mathscr{L}\left(C_{2}^{\mathscr{L}}\left(g, g^{\prime \prime}\right)+C_{2}^{\mathscr{L}}\left(g^{\prime}, g^{\prime \prime}\right)\right)$ to the following composition of 1-isomorphisms:

$$
\begin{aligned}
\mathscr{L}_{g g^{\prime} g^{\prime \prime}} & \simeq \mathscr{L}_{g}+\mathscr{L}_{g^{\prime} g^{\prime \prime}} \simeq \mathscr{L}_{g}+\left(\mathscr{L}_{g^{\prime}}+\mathscr{L}_{g^{\prime \prime}}\right) \simeq \mathscr{L}_{g}+\left(\mathscr{L}_{g^{\prime \prime}}+\mathscr{L}_{g^{\prime}}\right) \simeq \mathscr{L}_{g}+\mathscr{L}_{g^{\prime \prime} g^{\prime}} \simeq \mathscr{L}_{g g^{\prime \prime} g^{\prime}} \\
& \simeq \mathscr{L}_{g g^{\prime \prime}}+\mathscr{L}_{g^{\prime}} \simeq\left(\mathscr{L}_{g}+\mathscr{L}_{g^{\prime \prime}}\right)+\mathscr{L}_{g^{\prime}} \simeq\left(\mathscr{L}_{g^{\prime \prime}}+\mathscr{L}_{g}\right)+\mathscr{L}_{g^{\prime}} \simeq \mathscr{L}_{g g^{\prime \prime}}+\mathscr{L}_{g^{\prime}} \simeq \mathscr{L}_{g g^{\prime} g^{\prime \prime}}
\end{aligned}
$$

By the definition of the central extension of $G$ by $\mathscr{P}$ (see diagram (2-17)), there is a canonical 2-isomorphism from the above composition of 1-isomorphisms to the following composition of 1-isomorphisms

$$
\begin{aligned}
\mathscr{L}_{g g^{\prime} g^{\prime \prime}} & \simeq \mathscr{L}_{g}+\mathscr{L}_{g^{\prime} g^{\prime \prime}} \simeq \mathscr{L}_{g}+\left(\mathscr{L}_{g^{\prime}}+\mathscr{L}_{g^{\prime \prime}}\right) \simeq \mathscr{L}_{g}+\left(\mathscr{L}_{g^{\prime \prime}}+\mathscr{L}_{g^{\prime}}\right) \\
& \simeq\left(\mathscr{L}_{g}+\mathscr{L}_{g^{\prime \prime}}\right)+\mathscr{L}_{g^{\prime}} \simeq\left(\mathscr{L}_{g^{\prime \prime}}+\mathscr{L}_{g}\right)+\mathscr{L}_{g^{\prime}} \simeq \mathscr{L}_{g g^{\prime \prime}}+\mathscr{L}_{g^{\prime}} \simeq \mathscr{L}_{g g^{\prime} g^{\prime \prime}} .
\end{aligned}
$$

From the hexagon axiom for 1-morphisms in the category $B \mathscr{P}$ (see diagram (2-13)) we have that the 1-isomorphism which is the composition of the 1-isomorphisms above is equal to the 1-isomorphism that is the composition of the 1-isomorphisms

$$
\begin{aligned}
\mathscr{L}_{g g^{\prime} g^{\prime \prime}} & \simeq \mathscr{L}_{g}+\mathscr{L}_{g^{\prime} g^{\prime \prime}} \simeq \mathscr{L}_{g}+\left(\mathscr{L}_{g^{\prime}}+\mathscr{L}_{g^{\prime \prime}}\right) \simeq\left(\mathscr{L}_{g}+\mathscr{L}_{g^{\prime}}\right)+\mathscr{L}_{g^{\prime \prime}} \\
& \simeq \mathscr{L}_{g^{\prime \prime}}+\left(\mathscr{L}_{g}+\mathscr{L}_{g^{\prime}}\right) \simeq\left(\mathscr{L}_{g^{\prime \prime}}+\mathscr{L}_{g}\right)+\mathscr{L}_{g^{\prime}} \simeq \mathscr{L}_{g g^{\prime \prime}}+\mathscr{L}_{g^{\prime}} \simeq \mathscr{L}_{g g^{\prime} g^{\prime \prime}} .
\end{aligned}
$$

By the "functoriality" of the commutativity constraints in the category $B \mathscr{P}$ (see axiom-diagram (2-14)), the 1-isomorphism that is the composition of the 1-isomorphisms above is equal to the one that is the composition of the 1-isomorphisms

$$
\begin{aligned}
\mathscr{L}_{g g^{\prime} g^{\prime \prime}} & \simeq \mathscr{L}_{g}+\mathscr{L}_{g^{\prime} g^{\prime \prime}} \simeq \mathscr{L}_{g}+\left(\mathscr{L}_{g^{\prime}}+\mathscr{L}_{g^{\prime \prime}}\right) \simeq\left(\mathscr{L}_{g}+\mathscr{L}_{g^{\prime}}\right)+\mathscr{L}_{g^{\prime \prime}} \simeq \mathscr{L}_{g g^{\prime}}+\mathscr{L}_{g^{\prime \prime}} \\
& \simeq \mathscr{L}_{g^{\prime \prime}}+\mathscr{L}_{g g^{\prime}} \simeq \mathscr{L}_{g^{\prime \prime}}+\left(\mathscr{L}_{g}+\mathscr{L}_{g^{\prime}}\right) \simeq\left(\mathscr{L}_{g^{\prime \prime}}+\mathscr{L}_{g}\right)+\mathscr{L}_{g^{\prime}} \simeq \mathscr{L}_{g g^{\prime \prime}}+\mathscr{L}_{g^{\prime}} \simeq \mathscr{L}_{g g^{\prime} g^{\prime \prime}} .
\end{aligned}
$$

Again, by the definition of the central extension of $G$ by $\mathscr{P}$ (see diagram (2-17), which we apply twice now), there is a canonical 2-isomorphism from the composition of 1-isomorphisms above to the composition of 1-isomorphisms

$$
\mathscr{L}_{g g^{\prime} g^{\prime \prime}} \simeq \mathscr{L}_{g g^{\prime}}+\mathscr{L}_{g^{\prime \prime}} \simeq \mathscr{L}_{g^{\prime \prime}}+\mathscr{L}_{g g^{\prime}} \simeq \mathscr{L}_{g g^{\prime} g^{\prime \prime}},
$$


which is canonically isomorphic to $\mathscr{L}\left(C_{2}^{\mathscr{L}}\left(g g^{\prime}, g^{\prime \prime}\right)\right)$.

Let us write down a diagram which will represent the above 2-isomorphisms. To simplify the notation, we will denote the 2-commutative diagram (2-17) by

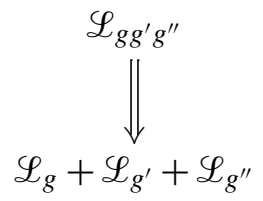

Then, the 2-isomorphism $\mathscr{L}\left(C_{2}^{\mathscr{L}}\left(g g^{\prime}, g^{\prime \prime}\right)\right) \simeq \mathscr{L}\left(C_{2}^{\mathscr{L}}\left(g, g^{\prime \prime}\right)\right)+\mathscr{L}\left(C_{2}^{\mathscr{L}}\left(g^{\prime}, g^{\prime \prime}\right)\right)$ is represented by the diagram

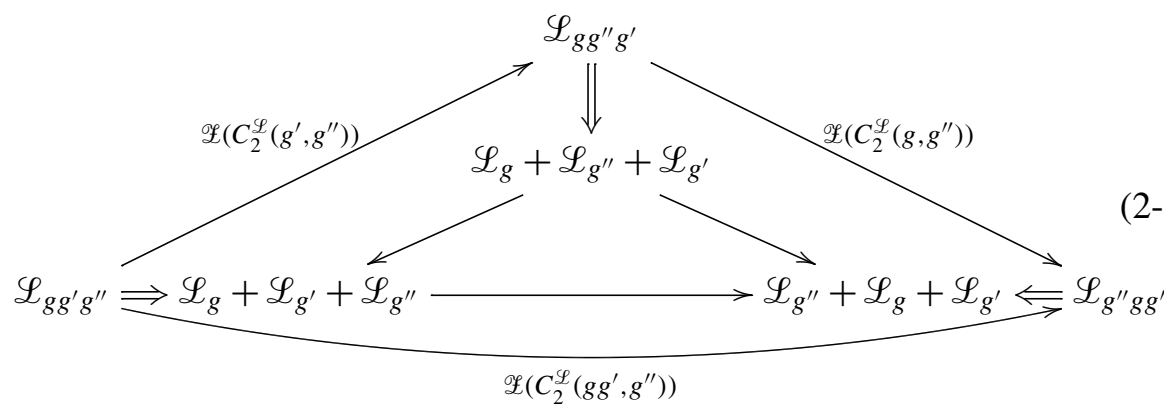

To check all the compatibility conditions between these canonical isomorphisms we generalize the proof of Lemma-Definition 2.5. We construct a 2-category $H_{\mathscr{L}}$ whose objects are objects from categories given by all expressions

$$
\left.\mathscr{L}_{g_{1}}+\cdots+\mathscr{L}_{g_{k}}:=\left(\cdots\left(\mathscr{L}_{g_{1}}+\mathscr{L}_{g_{2}}\right)+\mathscr{L}_{g_{3}}\right)+\cdots\right)+\mathscr{L}_{g_{k}}, \text { where } g_{i} \in G ;
$$

the 1-morphisms in $H_{\mathscr{L}}$ are defined as follows:

$$
\begin{aligned}
& \operatorname{Hom}_{H_{\mathscr{L}}}\left(\mathscr{L}_{g_{i_{1}}}+\cdots+\mathscr{L}_{g_{i_{k}}}, \mathscr{L}_{g_{j_{1}}}+\cdots+\mathscr{L}_{g_{j_{l}}}\right) \\
& =\left\{\begin{array}{cl}
\varnothing & \text { if } g_{i_{1}} \ldots g_{i_{k}} \neq g_{j_{1}} \ldots g_{j_{l}}, \\
\operatorname{Hom}_{B \mathscr{P}}\left(\mathscr{L}_{g_{i_{1}}}+\cdots+\mathscr{L}_{g_{i_{k}}}, \mathscr{L}_{g_{j_{1}}}+\cdots+\mathscr{L}_{g_{j_{l}}}\right) & \text { if } g_{i_{1}} \ldots g_{i_{k}}=g_{j_{1}} \ldots g_{j_{l}},
\end{array}\right.
\end{aligned}
$$

and the 2-morphisms in the 2-category $H_{\mathscr{L}}$ come from the 2-morphisms of category $B \mathscr{P}$. The category $H_{\mathscr{L}}$ is a monoidal group-like 2-groupoid (or a 2-gr-category), see [Breen 1994, §8], where the monoidal structure on $H_{\mathscr{L}}$ is given in an obvious way by using the associativity constraints in the category $B \mathscr{P}$ and the pentagon diagram (2-9). We have $\pi_{0}\left(H_{\mathscr{L}}\right)=G$. We consider the $\mathscr{P}$-torsor $\mathscr{E}^{\mathscr{L}}$ on $Z_{2}$ which is the commutator of $H_{\mathscr{L}}$. (See [Breen 1999, §8]. $\left.{ }^{8}\right)$ The fiber of $\mathscr{C}^{\mathscr{L}}$ over $\left(g_{1}, g_{2}\right) \in Z_{2}$

${ }^{8} \mathrm{~L}$. Breen assumed for simplicity in loc. cit. that the group $\pi_{1}$ of a 2-gr-category is equal to 0 . We have $\pi_{1}\left(H_{\mathscr{L}}\right) \neq 0$, but the constructions and its properties which we need remain true in our situation. 
is the $\mathscr{P}$-torsor

$$
\mathscr{\mathscr { E }}_{g_{1}, g_{2}}^{\mathscr{L}}=\operatorname{Hom}_{H_{\mathscr{L}}}\left(\mathscr{L}_{g_{1}}+\mathscr{L}_{g_{2}}, \mathscr{L}_{g_{2}}+\mathscr{L}_{g_{1}}\right) \text {. }
$$

The $\mathscr{P}$-torsor $\mathscr{C}^{\mathscr{L}}$ on $Z_{2}$ has a natural structure of a weak $(2,2)$-extension (see [Breen 1999, Proposition 8.1]), i.e., there are partial composition (group) laws on $\mathscr{E}^{\mathscr{L}}$ which are compatible (see diagrams (7.1), (7.3) in loc. cit). Now the commutativity constraints $C$ from $B \mathscr{P}$ give a trivialization of $\mathscr{P}$-torsor $\mathscr{E}^{\mathscr{L}}$ on $Z_{2}$ which is compatible with partial composition laws on $\mathscr{E}^{\mathscr{L}}$, i.e., "bimultiplicative". (The compatibility of this trivialization with composition laws follows at once from definition of partial composition laws on $\mathscr{C}^{\mathscr{L}}$ and hexagon diagram (2-13). See also the discussion in the end of [Breen 1999, §8] regarding the braiding structure

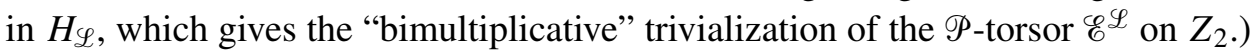
The other trivialization of the $\mathscr{P}$-torsor $\mathscr{E}^{\mathscr{L}}$ on $Z_{2}$ which is compatible with partial composition laws on $\mathscr{E}^{\mathscr{L}}$ is obtained as the composition of the following two equivalences from definition of $\mathscr{L}$ :

$$
S_{\mathscr{L}_{1}, \mathscr{L}_{g_{2}}}: \mathscr{L}_{g_{1}}+\mathscr{L}_{g_{2}} \simeq \mathscr{L}_{g_{1} g_{2}}=\mathscr{L}_{g_{2} g_{1}} \simeq \mathscr{L}_{g_{2}}+\mathscr{L}_{g_{1}} .
$$

Now the difference between the first trivialization and the second trivialization of the $\mathscr{P}$-torsor $\mathscr{E}^{\mathscr{L}}$ on $Z_{2}$ coincides with $C_{2}^{\mathscr{L}}$, which is, thus, a bimultiplicative homomorphism, because both trivializations are "bimultiplicative".

We have shown that $C_{2}^{\mathscr{L}}: Z_{2} \rightarrow \mathscr{P}$ is a bimultiplicative 1-morphism. One readily checks from the above constructions that this is antisymmetric from $Z_{2}$ to $\mathscr{P}$, since $C^{2}=\mathrm{id}$.

Remark 2.14. If $\mathscr{P}=B A$, then the construction of $C_{2}^{\mathscr{L}}$ given above is equivalent to the construction of the commutator category of the central extension $-\mathscr{L}$ introduced in [Deligne 1991].

We also have the following categorical analog of Corollary 2.9. First, let us remark that if $f_{1}, f_{2}: Z_{2} \rightarrow \mathscr{P}$ are two bimultiplicative homomorphisms, one can define $f_{1}+f_{2}$, which is again a bimultiplicative homomorphism, in the same way as defining the Picard structure on $H^{1}(B G, \mathscr{P})$.

Lemma 2.15. For any two central extensions $\mathscr{L}$ and $\mathscr{L}^{\prime}$ of $G$ by $\mathscr{P}$ there is a natural bimultiplicative 2-isomorphism (i.e., it respects the bimultiplicative structure) between bimultiplicative 1-morphisms $C_{2}^{\mathscr{L}+\mathscr{L}^{\prime}}$ and $C_{2}^{\mathscr{L}}+C_{2}^{\mathscr{L}^{\prime}}$.

Proof. Recall that we have the following canonical 1-isomorphism

$$
\mathscr{L}: \mathscr{P} \rightarrow \operatorname{Hom}\left(\mathscr{L}_{g g^{\prime}}+\mathscr{L}_{g g^{\prime}}^{\prime}, \mathscr{L}_{g g^{\prime}}+\mathscr{L}_{g g^{\prime}}^{\prime}\right) .
$$

We construct a canonical isomorphism

$$
\mathscr{L}\left(C_{2}^{\mathscr{L}+\mathscr{L}^{\prime}}\left(g, g^{\prime}\right)\right) \simeq \mathscr{L}\left(C_{2}^{\mathscr{L}}\left(g, g^{\prime}\right)+C_{2}^{\mathscr{L}^{\prime}}\left(g, g^{\prime}\right)\right)
$$


for any $\left(g, g^{\prime}\right) \in Z_{2}$ as follows. By definition, $\mathscr{L}\left(C_{2}^{\mathscr{L}+\mathscr{L}^{\prime}}\left(g, g^{\prime}\right)\right)$ is canonically 2-isomorphic to the composition of 1-morphisms

$$
\begin{aligned}
& \left(\mathscr{L}+\mathscr{L}^{\prime}\right)_{g g^{\prime}}=\mathscr{L}_{g g^{\prime}}+\mathscr{L}_{g g^{\prime}}^{\prime} \simeq\left(\mathscr{L}_{g}+\mathscr{L}_{g^{\prime}}\right)+\left(\mathscr{L}_{g}^{\prime}+\mathscr{L}_{g^{\prime}}^{\prime}\right) \simeq\left(\mathscr{L}_{g}+\mathscr{L}_{g}^{\prime}\right)+\left(\mathscr{L}_{g^{\prime}}+\mathscr{L}_{g^{\prime}}^{\prime}\right) \\
& =\left(\mathscr{L}+\mathscr{L}^{\prime}\right)_{g}+\left(\mathscr{L}+\mathscr{L}^{\prime}\right)_{g^{\prime}} \simeq\left(\mathscr{L}+\mathscr{L}^{\prime}\right)_{g^{\prime}}+\left(\mathscr{L}+\mathscr{L}^{\prime}\right)_{g} \simeq\left(\mathscr{L}_{g^{\prime}}+\mathscr{L}_{g^{\prime}}^{\prime}\right)+\left(\mathscr{L}_{g}+\mathscr{L}_{g}^{\prime}\right) \\
& \simeq\left(\mathscr{L}_{g^{\prime}}+\mathscr{L}_{g}\right)+\left(\mathscr{L}_{g^{\prime}}^{\prime}+\mathscr{L}_{g}^{\prime}\right) \simeq \mathscr{L}_{g^{\prime} g}+\mathscr{L}_{g^{\prime} g}^{\prime}=\left(\mathscr{L}+\mathscr{L}^{\prime}\right)_{g^{\prime} g}=\left(\mathscr{L}+\mathscr{L}^{\prime}\right)_{g g^{\prime}} .
\end{aligned}
$$

Using the functoriality of commutativity constraints, i.e., applying diagram (2-14) twice, and using the following commutative diagram (which is written without associativity constraints)

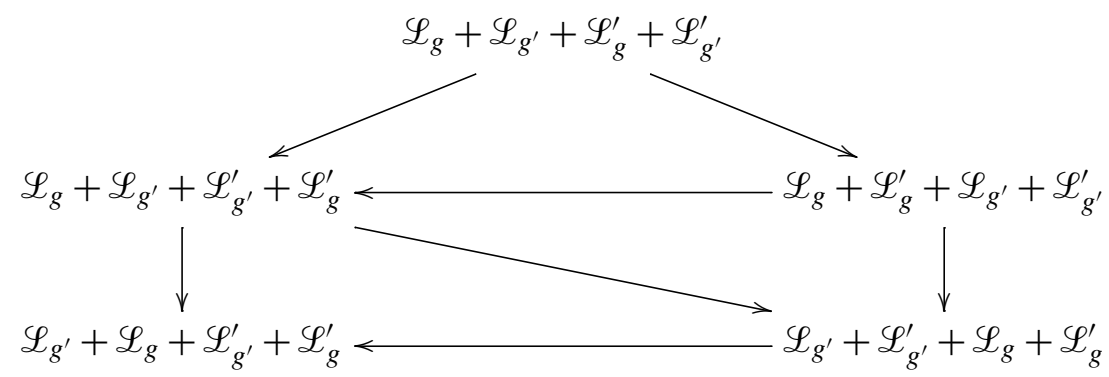

(to obtain the correct diagram we have to replace every triangle in this diagram by a hexagon coming from (2-13)), we obtain that the composition of 1-morphisms in (2-30) is equal to the composition of 1-morphisms

$$
\begin{aligned}
\mathscr{L}_{g g^{\prime}}+\mathscr{L}_{g g^{\prime}}^{\prime} & \simeq\left(\mathscr{L}_{g}+\mathscr{L}_{g^{\prime}}\right)+\left(\mathscr{L}_{g}^{\prime}+\mathscr{L}_{g^{\prime}}^{\prime}\right) \\
& \simeq\left(\mathscr{L}_{g^{\prime}}+\mathscr{L}_{g}\right)+\left(\mathscr{L}_{g^{\prime}}^{\prime}+\mathscr{L}_{g}^{\prime}\right) \simeq \mathscr{L}_{g^{\prime} g}+\mathscr{L}_{g^{\prime} g}^{\prime}=\mathscr{L}_{g g^{\prime}}+\mathscr{L}_{g g^{\prime}}^{\prime},
\end{aligned}
$$

which is, by definition, 2-isomorphic to $\mathscr{L}\left(C_{2}^{\mathscr{L}}\left(g, g^{\prime}\right)+C_{2}^{\mathscr{L}^{\prime}}\left(g, g^{\prime}\right)\right)$.

To complete the proof, we need to show that the diagram

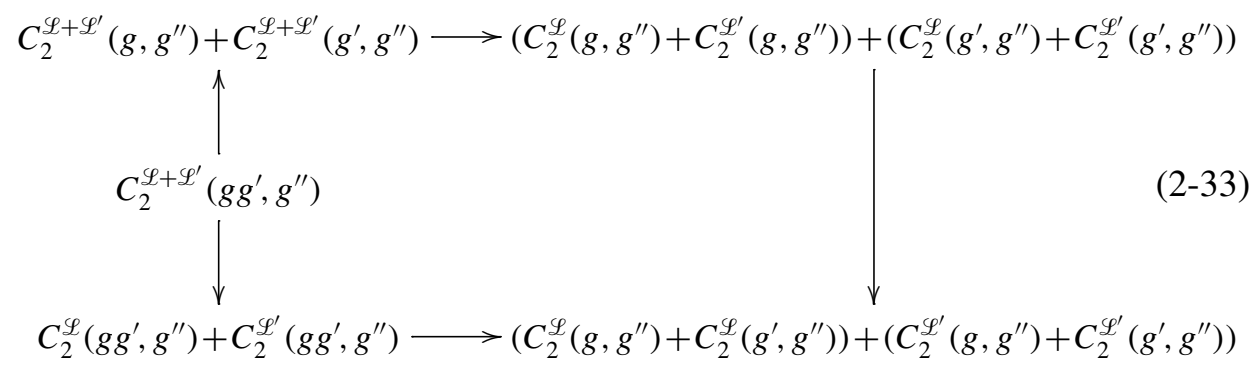

and a similar diagram involving $C_{2}^{\mathscr{L}+\mathscr{L}^{\prime}}\left(g, g^{\prime} g^{\prime \prime}\right)$ are commutative. To prove this, let us recall that the 2-isomorphism $C_{2}^{\mathscr{L}}\left(g g^{\prime}, g^{\prime \prime}\right) \simeq C_{2}^{\mathscr{L}}\left(g, g^{\prime \prime}\right)+C_{2}^{\mathscr{L}}\left(g^{\prime}, g^{\prime \prime}\right)$ is the composition of the 2-isomorphisms

$\mathscr{L}\left(C_{2}^{\mathscr{L}}\left(g, g^{\prime \prime}\right)+C_{2}^{\mathscr{L}}\left(g^{\prime}, g^{\prime \prime}\right)\right) \rightarrow(2-23) \rightarrow(2-24) \rightarrow \cdots \rightarrow(2-27) \rightarrow \mathscr{L}\left(C_{2}^{\mathscr{L}}\left(g g^{\prime}, g^{\prime \prime}\right)\right)$. 
Let us denote the 1-isomorphism (2-23) for $\mathscr{L}$ (resp. $\mathscr{L}^{\prime}$, resp. $\left.\mathscr{L}+\mathscr{L}^{\prime}\right)$ as $(2-23) \mathscr{L}$ (resp. $(2-23)_{\mathscr{L}}$, resp. $\left.(2-23)_{\mathscr{L}+\mathscr{L}^{\prime}}\right)$ and etc. Then it is readily checked that there exists a canonical 2-isomorphism

$$
(2-23)_{\mathscr{L}}+(2-23)_{\mathscr{L}^{\prime}} \simeq(2-23)_{\mathscr{L}+\mathscr{L}^{\prime}}
$$

between corresponding 1-isomorphisms $\mathscr{L}_{g g^{\prime} g^{\prime \prime}}+\mathscr{L}_{g g^{\prime} g^{\prime \prime}}^{\prime} \rightarrow \mathscr{L}_{g g^{\prime} g^{\prime \prime}}+\mathscr{L}_{g g^{\prime} g^{\prime \prime}}$, and canonical 2-isomorphisms for (2-24)-(2-27) such that the diagram

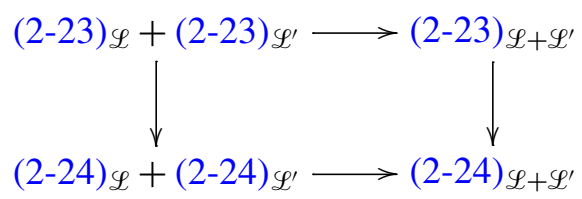

and similar diagrams for (2-24)-(2-27) commute. In addition, the following diagrams commute:

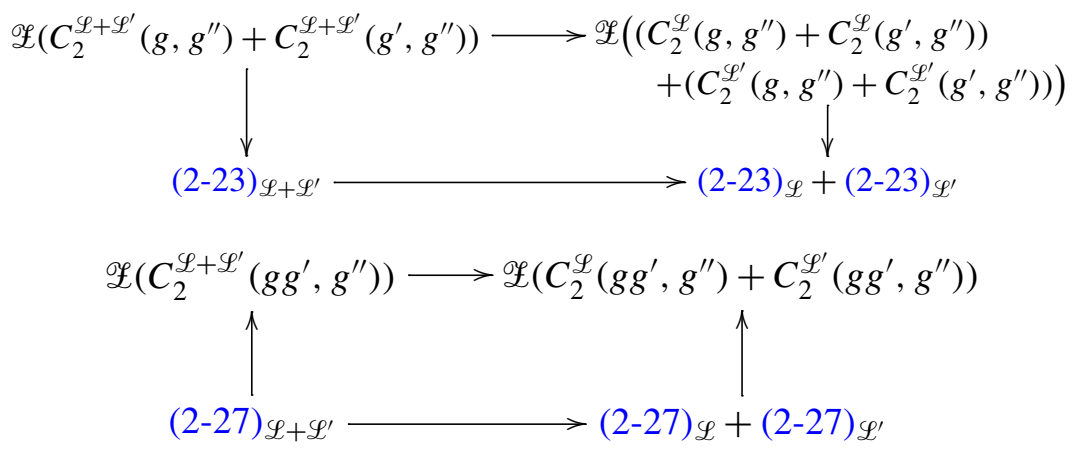

These facts together imply the commutativity of diagram (2-33).

Fix $g \in G$, the induced map $Z_{G}(g) \rightarrow \mathscr{P}$ given by $g^{\prime} \mapsto C_{2}^{\mathscr{L}}\left(g, g^{\prime}\right)$ is denoted by $C_{g}^{\mathscr{L}}$. The bimultiplicativity of $C_{2}^{\mathscr{L}}$ implies that $C_{g}^{\mathscr{L}}$ is an object in $H^{1}\left(B Z_{G}(g), \mathscr{P}\right)$. It is easy to see from the definition the following lemma:

Lemma 2.16. (i) If two central extensions $\mathscr{L}$ and $\mathscr{L}^{\prime}$ of $G$ by $\mathscr{P}$ are isomorphic in $H^{2}(B G, \mathscr{P})$, then for any $g$ the induced two homomorphisms $C_{g}^{\mathscr{L}}$ and $C_{g}^{\mathscr{L}^{\prime}}$ are isomorphic in $H^{1}\left(B Z_{G}(g), \mathscr{P}\right)$.

(ii) $C_{g}^{\mathscr{P}}$ is the trivial homomorphism for any $g \in G$.

Let $Z_{3} \subset G \times G \times G$ be the subset of pairwise commuting elements.

Proposition 2.17. The map

$$
C_{3}^{\mathscr{L}}: Z_{3} \rightarrow \pi_{1}(\mathscr{P})
$$

defined by

$$
C_{3}^{\mathscr{L}}\left(g, g^{\prime}, g^{\prime \prime}\right):=\operatorname{Comm}\left(C_{g}^{\mathscr{L}}\right)\left(g^{\prime}, g^{\prime \prime}\right) .
$$

is an antisymmetric trimultiplicative homomorphism from $Z_{3}$ to $\pi_{1}(\mathscr{P})$. 
Proof. We check the trimultiplicativity of the map $C_{3}^{\mathscr{L}}$. The multiplicativity of this map with respect to $g^{\prime}$ or $g^{\prime \prime}$ follows from Lemma-Definition 2.5. Multiplicativity with respect to $g$ follows from Lemma-Definition 2.13 and Corollary 2.9.

The hard part now is to prove that the map $C_{3}^{\mathscr{L}}$ is antisymmetric. Let us write $C_{2}$ instead of $C_{2}^{\mathscr{L}}$, and $C_{3}$ instead of $C_{3}^{\mathscr{L}}$ for simplicity. Let $\left(g, g^{\prime}, g^{\prime \prime}\right) \in Z_{3}$. First of all, let us observe that by definition, there is a canonical isomorphism

$$
C_{2}\left(g, g^{\prime} g^{\prime \prime}\right)+C_{2}\left(g^{\prime}, g^{\prime \prime}\right) \simeq C_{2}\left(g^{\prime}, g^{\prime \prime}\right)+C_{2}\left(g, g^{\prime \prime} g^{\prime}\right)
$$

induced by the 2-commutative diagram

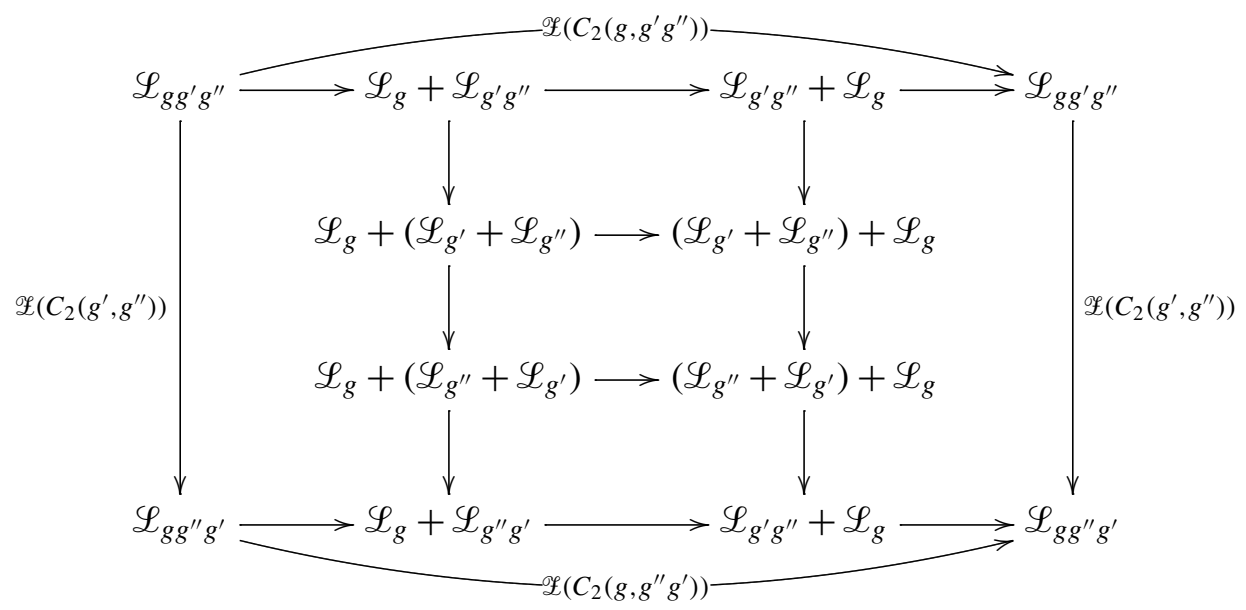

The following lemma can be checked using the definition of $B \mathscr{P}$.

Lemma 2.18. The isomorphism (2-34) is the same as the commutativity constraint in $\mathscr{P}$.

Now, there are two isomorphisms between $\left(C_{2}\left(g, g^{\prime}\right)+C_{2}\left(g, g^{\prime \prime}\right)\right)+C_{2}\left(g^{\prime}, g^{\prime \prime}\right)$ and $C_{2}\left(g^{\prime}, g^{\prime \prime}\right)+\left(C_{2}\left(g, g^{\prime \prime}\right)+C_{2}\left(g, g^{\prime}\right)\right)$. Namely, the first isomorphism is obtained by the associativity and commutativity constraints in $\mathscr{P}$. (Recall that such isomorphism is unique by Mac Lane's coherence theorem for Picard category.) The second isomorphism is

$$
\begin{gathered}
\left(C_{2}\left(g, g^{\prime}\right)+C_{2}\left(g, g^{\prime \prime}\right)\right)+C_{2}\left(g^{\prime}, g^{\prime \prime}\right) \simeq C_{2}\left(g, g^{\prime} g^{\prime \prime}\right)+C_{2}\left(g^{\prime}, g^{\prime \prime}\right) \stackrel{(2-34)}{\simeq} \\
C_{2}\left(g^{\prime}, g^{\prime \prime}\right)+C_{2}\left(g, g^{\prime \prime} g^{\prime}\right) \simeq C_{2}\left(g^{\prime}, g^{\prime \prime}\right)+\left(C_{2}\left(g, g^{\prime \prime}\right)+C_{2}\left(g, g^{\prime}\right)\right) .
\end{gathered}
$$

By the lemma, the difference between these two isomorphisms is $C_{3}\left(g, g^{\prime}, g^{\prime \prime}\right)$. If we recall the definition of $C_{2}\left(g, g^{\prime} g^{\prime \prime}\right) \simeq C_{2}\left(g, g^{\prime}\right)+C_{2}\left(g, g^{\prime \prime}\right)$ by (2-29), we see that the isomorphism (2-35) can be represented by the diagram 


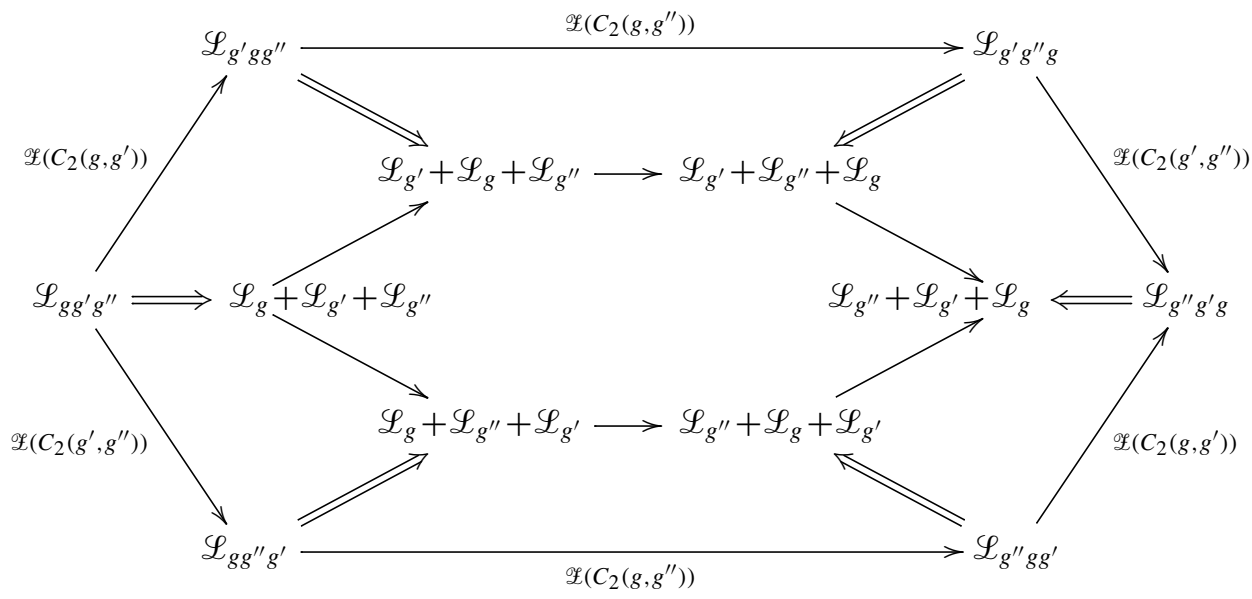

This diagram clearly implies that $C_{3}\left(g, g^{\prime}, g^{\prime \prime}\right)=C_{3}\left(g^{\prime}, g^{\prime \prime}, g\right)$. This, together with the fact that $C_{3}\left(g, g^{\prime}, g^{\prime \prime}\right)=-C_{3}\left(g, g^{\prime \prime}, g^{\prime}\right)$ (because the map $\operatorname{Comm}\left(C_{g}^{\mathscr{L}}\right)$ is antisymmetric), implies that $C_{3}$ is antisymmetric.

Corollary 2.19. (i) If two central extensions $\mathscr{L}$ and $\mathscr{L}^{\prime}$ of $G$ by $\mathscr{P}$ are isomorphic in $H^{2}(B G, \mathscr{P})$, then $C_{3}^{\mathscr{L}}=C_{3}^{\mathscr{L}^{\prime}}$.

(ii) $C_{3}^{\mathscr{P}}$ is trivial.

Corollary 2.20. For any two central extensions $\mathscr{L}$ and $\mathscr{L}^{\prime}$ of $G$ by $\mathscr{P}$ we have

$$
C_{3}^{\mathscr{L}+\mathscr{L}^{\prime}}=C_{3}^{\mathscr{L}}+C_{3}^{\mathscr{L}^{\prime}}
$$

Proof. This follows from Lemma 2.15, Corollary 2.9 and the definition of $C_{3}$.

Remark 2.21. If $\mathscr{P}=B A$, where $A$ is an abelian group, then a central extension $\mathscr{L}$ of a group $G$ by the Picard groupoid $\mathscr{P}$ is a $g r$-category such that these $g r$ categories are classified by the group $H^{3}(G, A)$ with the trivial $G$-module $A$. In this case the map $C_{3}^{\mathscr{L}}$ coincides with the symmetrization of corresponding 3-cocycle; see [Breen 1999, §4]. (This follows from Remarks 2.14, 2.8 and [Osipov 2003, Proposition 10].)

\section{Tate vector spaces}

3A. The category of Tate vector spaces. We first review the definition of Tate vector spaces, following [Osipov 2007; Arkhipov and Kremnizer 2010]. Let us fix a base field $k$.

Recall that Beilinson [1987] associates to an exact category $\mathscr{E}$ in the sense of

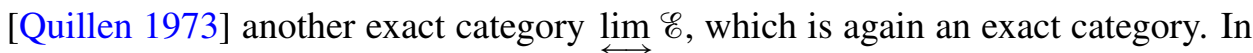
nowadays terminology, this is the category of locally compact objects of $\mathscr{E}$. 
For an exact category $\mathscr{E}$, let $\hat{\mathscr{E}}$ denote the category of left exact additive contravariant functors from the category $\mathscr{E}$ to the category of abelian groups. This is again an exact category (in fact an abelian category), in which arbitrary small colimits exist. The Yoneda embedding $h: \mathscr{E} \rightarrow \hat{\mathscr{E}}$ is exact. Then the category $\operatorname{Ind}(\mathscr{E})$ of (strict) ind-objects of $\mathscr{E}$, is the full subcategory of $\hat{\mathscr{E}}$ consisting of objects of the form $\lim _{i \in I} h\left(X_{i}\right)$, where $I$ is a filtered small category, and $X_{i} \in \mathscr{E}$, such that for $i \rightarrow j$ in $I$, the map $X_{i} \rightarrow X_{j}$ is an admissible monomorphism. This category is a natural exact category. Likewise, one can define $\operatorname{Pro}(\mathscr{E})$ as $\operatorname{Ind}(\mathscr{E} \circ)^{\text {op }}$.

Definition 3.1. Let $\mathscr{E}$ be an exact category. Then $\lim _{\longleftrightarrow} \mathscr{E}$ is the full subcategory of Pro(Ind $(\mathscr{E}))$ consisting of objects that can be represented as

$$
\lim _{i \in I^{\text {op }}} \underset{j \in I}{\lim } h\left(X_{i j}\right)
$$

such that, for any $i \rightarrow i^{\prime}, j \rightarrow j^{\prime}$, the following diagram is cartesian (which automatically makes it cocartesian).

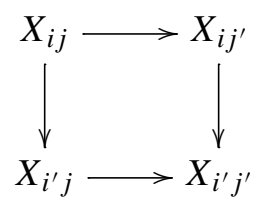

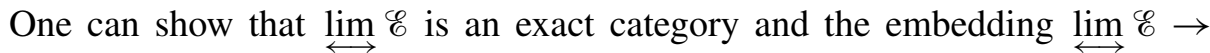

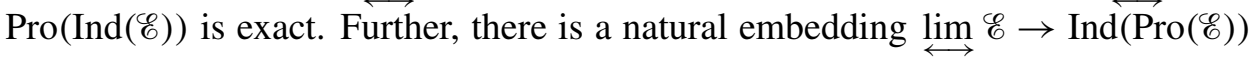
which is again exact. It is clear that the natural embedding $\overleftrightarrow{\operatorname{Ind}(\mathscr{E})} \rightarrow \operatorname{Pro}(\operatorname{Ind}(\mathscr{E}))$ lands in $\lim _{\longleftrightarrow} \mathscr{E}$, and similarly the natural embedding $\operatorname{Pro}(\mathscr{E}) \rightarrow \operatorname{Ind}(\operatorname{Pro}(\mathscr{E}))$ lands in $\lim _{\longleftrightarrow} \mathscr{E}$.

Definition 3.2. Define Tate $_{0}$ to be the category of finite dimensional vector spaces, together with its canonical exact category structure. Define Tate ${ }_{n}=\underset{\longleftrightarrow}{\lim }$ Tate $_{n-1}$, together with the canonical exact category structure given by Beilinson.

There is a canonical forgetful functor $F_{n}:$ Tate $_{n} \rightarrow$ Top, where $\mathscr{T}_{o p}$ denotes the category of topological vector spaces. As is shown in [Osipov 2007], the functor is fully faithful when $n=1$, but this is in general not the case when $n>1$.

Definition 3.3. Let $V$ be an object of Tate ${ }_{n}$. A lattice $L$ of $V$ is an object in Tate ${ }_{n}$ which actually belongs to Pro(Tate $\left.{ }_{n-1}\right)$, together with an admissible monomorphism $L \rightarrow V$ such that the object $V / L$ belongs to $\operatorname{Ind}\left(\right.$ Tate $\left._{n-1}\right)$. A colattice $L^{c}$ of $V$ is an object in Tate ${ }_{n}$ which actually belongs to Ind(Tate $\left.{ }_{n-1}\right)$, together with an addmissible monomorphism $L^{c} \rightarrow V$ such that the object $V / L^{c}$ belongs to Pro( Tate $\left._{n-1}\right)$. 
It is clear that if $L$ is a lattice of $V$ and $L^{c}$ is a colattice, then $L \cap L^{c}$ belongs to Tate $_{n-1}$.

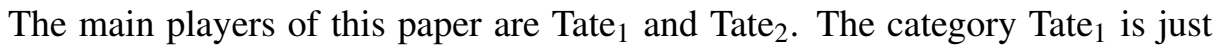
the category of locally linearly compact $k$-vector spaces. A typical object in Tate 1 is the field of formal Laurent series $k((t))$, that is, the field of fractions of the ring $k \llbracket t \rrbracket$. The field $k((t))$ is equipped with the standard topology, where the base of neighborhoods of zero consists of integer powers of the maximal ideal of $k \llbracket t \rrbracket$. The subspace $k \llbracket t \rrbracket$ is a lattice in $k((t))$ and $k\left[t^{-1}\right]$ is a colattice. Observe that $k[t] \subset k((t))$ is neither a lattice nor a colattice, because the subspace $k[t]$ is not closed in the topological space $k((t))$. Therefore the embedding $k[t] \hookrightarrow k((t))$ is not an admissible monomorphism, since any admissible (exact) triple in the category Tate $_{1}$ is of the form

$$
0 \longrightarrow V_{1} \longrightarrow V_{2} \longrightarrow V_{3} \longrightarrow 0,
$$

where the locally linearly compact vector space $V_{1}$ is a closed vector subspace in a locally linearly compact vector space $V_{2}$, and the locally linearly compact vector space $V_{3}$ has the quotient topology on the quotient vector space.

A typical object in Tate $_{2}$ is $k((t))((s))$, since

$$
k((t))((s))=\lim _{l \in \mathbb{Z}} \underset{m \leq l}{\lim } s^{m} k((t)) \llbracket s \rrbracket / s^{l} k((t)) \llbracket s \rrbracket={\underset{m \in \mathbb{Z}}{\longrightarrow}}_{l \geq m} \lim _{l} s^{m} k((t)) \llbracket s \rrbracket / s^{l} k((t)) \llbracket s \rrbracket,
$$

and $s^{m} k((t)) \llbracket s \rrbracket / s^{l} k((t)) \llbracket s \rrbracket$ is a locally linearly compact $k$-vector space.

The $k$-space $k((t)) \llbracket s \rrbracket$ is a lattice, and the $k$-space $k((t))\left[s^{-1}\right]$ is a colattice in the $k$-space $k((t))((s))$. As just mentioned above, it is not enough to regard them as topological vector spaces. On the other hand $k \llbracket t \rrbracket((s))$ is not a lattice in $k((t))((s))$ although the natural map $k \llbracket t \rrbracket((s)) \rightarrow k((t))((s))$ is an admissible monomorphism.

Remark 3.4. The category Tate ${ }_{n}$ coincides with the category of complete $C_{n^{-}}$ spaces from [Osipov 2007].

3B. Determinant theories of Tate vector spaces. We consider Tate ${ }_{0}$ as an exact category. Then det $:\left(\right.$ Tate $_{0}$, isom) $\rightarrow \mathscr{P} i c^{\mathbb{Z}}$ (see (2-6)) is a functor satisfying the following additional property: for each injective homomorphism $V_{1} \rightarrow V$ in the category Tate $_{0}$, there is a canonical isomorphism

$$
\operatorname{det}\left(V_{1}\right) \otimes \operatorname{det}\left(V / V_{1}\right) \simeq \operatorname{det}(V),
$$

such that:

(i) for $V_{1}=0$ (resp. $V_{1}=V$ ), equality (3-1) is the same as

$$
\ell_{0} \otimes \operatorname{det}(V) \simeq \operatorname{det}(V)
$$

resp.

$$
\operatorname{det}(V) \otimes \ell_{0} \simeq \operatorname{det}(V)
$$


where $\ell_{0}$ is the trivial $k$-line of degree zero.

(ii) For any diagram

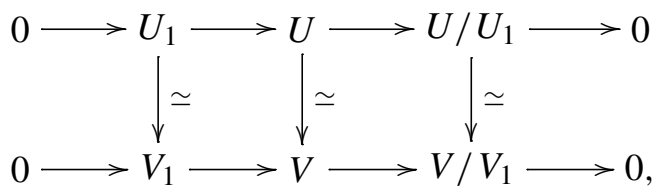

the following diagram is commutative:

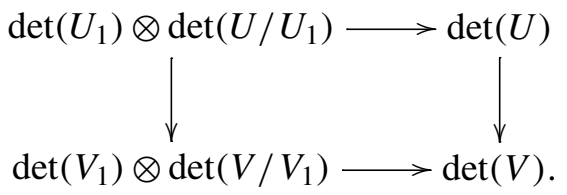

(iii) For any diagram

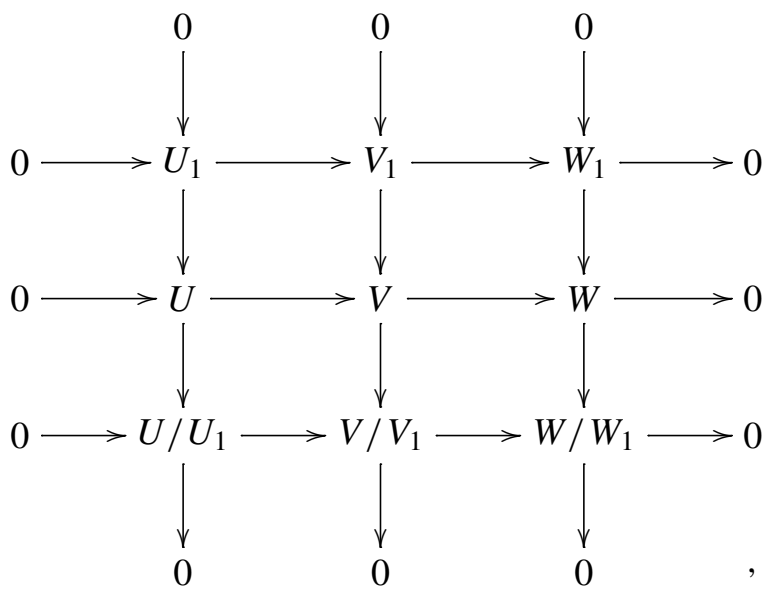

the following diagram is commutative:

$\left(\operatorname{det}\left(U_{1}\right) \otimes \operatorname{det}\left(U / U_{1}\right)\right) \otimes\left(\operatorname{det}\left(W_{1}\right) \otimes \operatorname{det}\left(W / W_{1}\right)\right) \longrightarrow \operatorname{det}(U) \otimes \operatorname{det}(W)$ ass. and comm. constraints

$\left(\operatorname{det}\left(U_{1}\right) \otimes \operatorname{det}\left(W_{1}\right)\right) \otimes\left(\operatorname{det}\left(U / U_{1}\right) \otimes \operatorname{det}\left(W / W_{1}\right)\right)$

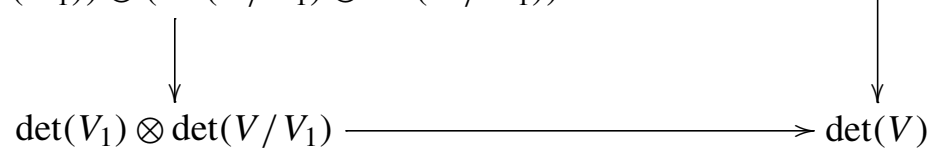

Definition 3.5. Let $\mathscr{P}$ be a Picard groupoid. A determinant functor from the category $\left(\right.$ Tate $_{0}$, isom) to $\mathscr{P}$ is a functor $D:\left(\right.$ Tate $_{0}$, isom) $\rightarrow \mathscr{P}$ together with isomorphisms (3-1) satisfying equalities and diagrams (3-2)-(3-7), where we have to change the notation det to $D$ everywhere in these formulas. 
The next proposition is obvious.

Proposition 3.6. Let $D:\left(\right.$ Tate $_{0}$, isom $) \rightarrow \mathscr{P}$ be a determinant functor. Then there is a 1-homomorphism of Picard groupoids $\tilde{D}: \mathscr{P} i c^{\mathbb{Z}} \rightarrow \mathscr{P}$ and a monoidal natural transformation $\varepsilon: \tilde{D} \circ$ det $\simeq D$. Furthermore, the pair $(\tilde{D}, \varepsilon)$ is unique up to a unique isomorphism.

Remark 3.7. All the above discussions are valid when one replaces $k$ by a noetherian commutative ring $A$, and replaces Tate ${ }_{0}$ by the category of finitely generated projective $A$-modules.

Next we turn to Tate ${ }_{1}$. The following result is fundamental and is due to Kapranov [2001] (but see also [Drinfeld 2006, §5.1-5.3]).

Proposition 3.8. There is a natural functor

$$
\text { Det }:\left(\text { Tate }_{1} \text {, isom }\right) \rightarrow B \mathscr{P i c} \text {, }
$$

and for each admissible monomorphism $\mathscr{V}_{1} \rightarrow \mathscr{V}$ there is a 1-isomorphism

$$
\operatorname{Det}\left(\mathscr{V}_{1}\right)+\operatorname{Det}\left(\mathscr{V} / \mathscr{V}_{1}\right) \rightarrow \operatorname{Det}(\mathscr{V})
$$

that coincides with the canonical 1-isomorphism $\mathscr{P}+\mathscr{D e t}(\mathscr{V}) \simeq \mathscr{D e t}(\mathscr{V})$ if $\mathscr{V}_{1}=0$ and with the canonical 1-isomorphism $\operatorname{Det}(\mathscr{V})+\mathscr{P} \simeq \operatorname{Det}(\mathscr{V})$ if $\mathscr{V}{ }_{1}=\mathscr{V}$.

For each admissible diagram (3-4) of 1-Tate vector spaces, the corresponding diagram (3-5) is commutative. For each admissible diagram (3-6) of 1-Tate vector spaces, there is a 2-isomorphism for the corresponding diagram (3-7).

Remark 3.9. Under conditions of Proposition 3.8, the 2-isomorphisms which appear from diagram (3-7) satisfy further compatibility conditions.

Proof. We recall the definition of a graded-determinantal theory $\Delta$ on a 1-Tate vector space $\mathscr{V}$. This is a rule that assign to every lattice $L \subset \mathscr{V}$ an object $\Delta(L)$ from $\mathscr{P} i c^{\mathbb{Z}}$ and to every lattices $L_{1} \subset L_{2} \subset \mathscr{V}$ an isomorphism

$$
\Delta_{L_{1}, L_{2}}: \Delta\left(L_{1}\right) \otimes \operatorname{det}\left(L_{2} / L_{1}\right) \longrightarrow \Delta\left(L_{2}\right)
$$

such that for any three lattices $L_{1} \subset L_{2} \subset L_{3} \subset \mathscr{V}$ the following diagram is commutative:

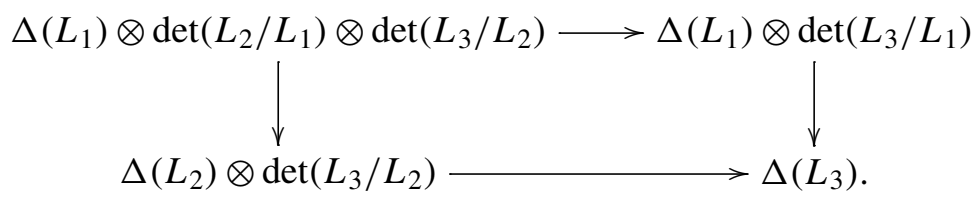

Let $\mathscr{D e t}(\mathscr{V})$ be the category of graded-determinantal theories on $\mathscr{V}$. This is a $\mathscr{P} i c^{\mathbb{Z}}$ torsor, where for any $x \in \mathscr{P} i c^{\mathbb{Z}}, \Delta \in \mathscr{D} e t(\mathscr{V})$, we have $(x+\Delta)(L):=x \otimes \Delta(L)$. 
Now for an admissible (exact) sequence

$$
0 \longrightarrow \mathscr{V}_{1} \longrightarrow \mathscr{V} \stackrel{\epsilon}{\longrightarrow} \mathscr{V} / \mathscr{V}_{1} \longrightarrow 0
$$

the 1-isomorphism (3-8) is constructed as

$$
\Delta(L):=\Delta_{1}\left(L \cap \mathscr{V}_{1}\right) \otimes \Delta_{2}(\epsilon(L)),
$$

where $L$ is a lattice in $\mathscr{V}, \Delta_{1} \in \operatorname{Det}(\mathscr{V} 1), \Delta_{2} \in \operatorname{Det}\left(\mathscr{V} / \mathscr{V}_{1}\right), \Delta \in \operatorname{Det}(\mathscr{V})$. (We used that the $k$-space $L \cap \mathscr{V}_{1}$ is a lattice in the 1-Tate vector space $\mathscr{V}_{1}$, and the $k$-space $\epsilon(L)$ is a lattice in the 1-Tate vector space $\left.\mathscr{V}_{3}\right)$.

We note that, by construction, $\mathscr{V} \mapsto \operatorname{Det}(\mathscr{V})$ is naturally a contravariant functor from the category (Tate ${ }_{1}$, isom) to the category $B \mathscr{P} i c^{\mathbb{Z}}$. To obtain the covariant functor we have to inverse arrows in the category (Tate ${ }_{1}$, isom).

\section{Applications to the case $G=\operatorname{GL}(k((t)))$ and $\operatorname{GL}(k((t))((s)))$}

4A. Tame symbols. Let us first review the tame symbols. Recall that if $K$ is a field with discrete valuation $v: K^{\times} \rightarrow \mathbb{Z}$, and $k$ denote its residue field, then there are so-called boundary maps for any $i \in \mathbb{N}$

$$
\partial_{i}: K_{i}^{M}(K) \longrightarrow K_{i-1}^{M}(k),
$$

where $K_{i}^{M}(F)$ denotes the $i$-th Milnor K-group of a field $F$. Recall also that for a field $F$, the $i$-th Milnor K-group $K_{i}^{M}(F)$ is the quotient of the abelian group $F^{\times} \otimes_{\mathbb{Z}} F^{\times} \otimes_{\mathbb{Z}} \cdots \otimes_{\mathbb{Z}} F^{\times}$modulo the so-called Steinberg relations. Then the tame symbol is defined as the composition of the maps

$$
\{\cdot, \cdot\}: K^{\times} \otimes_{\mathbb{Z}} K^{\times} \longrightarrow K_{2}^{M}(K) \stackrel{\partial_{2}}{\longrightarrow} K_{1}^{M}(k) \simeq k^{\times} .
$$

Explicitly, let $\pi \subset K$ be the maximal ideal. Then

$$
\{f, g\}=(-1)^{\nu(f) \nu(g)} \frac{f^{\nu(g)}}{g^{\nu(f)}} \bmod \pi
$$

Now, let $\mathbb{K}$ be a two-dimensional local field, whose residue field is denoted by $K$, whose residue field is $k$. Then we define the map

$$
\nu_{\mathbb{K}}: \mathbb{K}^{\times} \otimes_{\mathbb{Z}} \mathbb{K}^{\times} \longrightarrow K_{2}^{M}(\mathbb{K}) \stackrel{\partial_{2}}{\longrightarrow} K_{1}^{M}(K) \stackrel{\partial_{1}}{\longrightarrow} K_{0}^{M}(k) \simeq \mathbb{Z},
$$

and define the two-dimensional tame symbol as

$$
\{\cdot, \cdot, \cdot\}: \mathbb{K}^{\times} \otimes_{\mathbb{Z}} \mathbb{K}^{\times} \otimes_{\mathbb{Z}} \mathbb{K}^{\times} \longrightarrow K_{3}^{M}(\mathbb{K}) \stackrel{\partial_{3}}{\longrightarrow} K_{2}^{M}(K) \stackrel{\partial_{2}}{\longrightarrow} K_{1}^{M}(k) \simeq k^{\times} .
$$

We have the following explicit formulas for $\nu_{\mathbb{K}}$ and $\{\cdot, \cdot, \cdot\}$ (see [Osipov 2003]). Let $v_{1}: \mathbb{K} \rightarrow \mathbb{Z}$, and $\nu_{2}: K \rightarrow \mathbb{Z}$ be discrete valuations. Let $\pi_{\mathbb{}}$ be the maximal 
ideal of $\mathbb{K}, \pi_{K}$ be the maximal ideal of $K$. For an element $f \in \mathcal{O}_{\mathbb{K}}$, let $\bar{f}$ denote its residue class in $K$. Then

$$
\nu_{\mathbb{K}}(f, g)=v_{2}\left(\overline{\frac{f^{\nu_{1}(g)}}{g^{\nu_{1}(f)}}}\right)
$$

and

$$
\{f, g, h\}=\operatorname{sgn}(f, g, h) f^{\nu_{\nVdash}(g, h)} g^{\nu_{\nwarrow}(h, f)} h^{\nu_{\nwarrow}(f, g)} \quad \bmod \pi_{\mathbb{K}} \quad \bmod \pi_{K}
$$

where

$$
\operatorname{sgn}(f, g, h)=(-1)^{A},
$$

with

$$
\begin{aligned}
A=v_{\mathbb{K}}(f, g) \nu_{\mathbb{K}}(f, h)+v_{\mathbb{K}}(g, h) \nu_{\mathbb{K}}(g, f)+v_{\mathbb{K}}(h, f) v_{\mathbb{K}}(h, g) & \\
& +v_{\mathbb{S}}(f, g) v_{\mathbb{K}}(g, h) v_{\mathbb{K}}(h, f) .
\end{aligned}
$$

Remark 4.1. Originally one used another explicit formula for the sign of the twodimensional tame symbol. This other formula was introduced in [Parshin 1975].

It is easy to see that tame symbols $\{\cdot, \cdot\},\{\cdot, \cdot, \cdot\}$ and the map $\nu_{\mathbb{K}}$ are antisymmetric.

4B. The one-dimensional story. Let $\mathscr{V}$ be a 1-Tate vector space over $k$. The group of automorphisms of $\mathscr{V}$ in this category is denoted by GL( $\mathscr{V})$.

Proposition 4.2. There is a homomorphism $\mathscr{D e t} \mathrm{t}_{\mathbb{V}}: \mathrm{GL}(\mathscr{V}) \rightarrow \mathscr{P i c}{ }^{\mathbb{Z}}$, which is canonical up to a unique isomorphism in $H^{1}\left(B \mathrm{GL}(\mathscr{V}), \mathscr{P} i c^{\mathbb{Z}}\right)$.

Proof. According to Proposition 3.8, we have a homomorphism

$$
\mathrm{GL}(\mathscr{V}) \rightarrow \operatorname{Hom}_{\mathscr{P} i c^{\mathbb{Z}}}(\operatorname{Det}(\mathscr{V}), \operatorname{Det}(\mathscr{V})) \simeq \mathscr{P i c} c^{\mathbb{Z}}
$$

via $\mathscr{L}^{-1}$, where $\mathscr{L}: \mathscr{P} i c^{\mathbb{Z}} \rightarrow \operatorname{Hom}_{\mathscr{P} i c^{\mathbb{Z}}}(\mathscr{D} e t(\mathscr{V})$, $\mathscr{D e t}(\mathscr{V}))$ is a natural homomorphism from Section $2 \mathrm{C}$.

Choose $\mathscr{L} \subset \mathscr{V}$ a lattice. It follows from the proof of Proposition 3.8 that in concrete terms, one has to assign to $\operatorname{Det} v(g)$ the graded line

$$
\operatorname{det}(\mathscr{L} \mid g \mathscr{L}):=\operatorname{det}\left(\frac{g \mathscr{L}}{\mathscr{L} \cap g \mathscr{L}}\right) \otimes \operatorname{det}\left(\frac{\mathscr{L}}{\mathscr{L} \cap g \mathscr{L}}\right)^{-1},
$$

where $g \in \mathrm{GL}(\mathscr{V})$. Then, it is well-known that there is a canonical isomorphism

$$
\operatorname{det}\left(\mathscr{L} \mid g g^{\prime} \mathscr{L}\right) \simeq \operatorname{det}(\mathscr{L} \mid g \mathscr{L}) \otimes \operatorname{det}\left(g \mathscr{L} \mid g g^{\prime} \mathscr{L}\right) \simeq \operatorname{det}(\mathscr{L} \mid g \mathscr{L}) \otimes \operatorname{det}\left(\mathscr{L} \mid g^{\prime} \mathscr{L}\right),
$$

which is compatible with the associativity constraints in the category $\mathscr{P} i c^{\mathbb{Z}}$ (see, for example, [Frenkel and Zhu 2008, §1]). For different choice of $\mathscr{L}$, the resulting objects in $H^{1}\left(B G L(\mathscr{V}), \mathscr{P i} c^{\mathbb{Z}}\right)$ are isomorphic. 
We also have the following lemma, which easily follows from the construction of homomorphism Deto and the discussion in Section 3B (in particular the diagram $(3-7))$.

Lemma 4.3. If $0 \rightarrow \mathscr{V}^{\prime} \rightarrow \mathscr{V} \rightarrow \mathscr{V}^{\prime \prime} \rightarrow 0$ is a short exact sequence of 1-Tate vector spaces (recall that Tate $_{1}$ is an exact category). Let $P$ be the subgroup of GL( $\left.\mathscr{V}\right)$ that preserves this sequence, then there is a canonical 1-isomorphism Detw' + Detw" $\simeq$ Deto in $H^{1}\left(B P, \mathscr{P} i c^{\mathbb{Z}}\right)$.

Remark 4.4. The 1-homomorphism $F_{\mathscr{P} i c} \circ \mathscr{D}_{\mathrm{e} t_{\mathrm{V}}}: \mathrm{GL}(\mathscr{V}) \rightarrow \mathscr{P i c}$ is essentially constructed in [Arbarello et al. 1988]. However, the above lemma does not hold for this 1-homomorphism. This is the complication of the sign issues in that reference.

Now let $k^{\prime} / k$ be a finite extension and $K=k^{\prime}((t))$ be a local field with residue field $k^{\prime}$. Then $K$ has a natural structure as a 1-Tate vector space over $k$. Let $H=$ $K^{\times}$. The multiplication gives a natural embedding $H \subset \mathrm{GL}(K)$. The following proposition is from [Beilinson et al. 2002].

Proposition 4.5. If $f, g \in H$, then

$$
\operatorname{Comm}\left(\mathscr{D e t}_{K}\right)(f, g)=\mathrm{Nm}_{k^{\prime} / k}\{f, g\}^{-1}
$$

that is inverse to the tame symbol of $f$ and $g$.

Remark 4.6. Since the natural functor $F_{\mathscr{P} i c}$ is monoidal, the restriction to $H$ of the functor $F_{\mathscr{P} i c} \circ \mathscr{D e t}_{K}$ determines a homomorphism $H \rightarrow \mathscr{P} i c$. The commutator pairing $\operatorname{Comm}(f, g)$ constructed by this homomorphism is

$$
(-1)^{\operatorname{ord}(f) \operatorname{ord}(g)} \mathrm{Nm}_{k^{\prime} / k}\{f, g\}^{-1} .
$$

By Definition 3.3, a lattice $\mathscr{L}$ of $\mathscr{V}$ is a linearly compact open $k$-subspace of $\mathscr{V}$ such that $\mathscr{V} / \mathscr{L}$ is a discrete $k$-space. A colattice $\mathscr{L}^{c}$ is a $k$-subspace of $\mathscr{V}$ such that for any lattice $\mathscr{L}$, both $\mathscr{L}^{c} \cap \mathscr{L}$ and $\mathscr{V} /\left(\mathscr{L}^{c}+\mathscr{L}\right)$ are finite dimensional.

Lemma 4.7. Let $P \subset \mathrm{GL}(\mathscr{V})$ be a subgroup of $\mathrm{GL}(\mathscr{V})$ that preserves a lattice (or a colattice) in $\mathcal{V}$, then the homomorphism Det $\mathrm{V}$ is trivial on $P$.

Proof. Let $\mathscr{L} \subset \mathscr{V}$ be a lattice such that the group $P$ preserves it. We consider an exact sequence of 1-Tate vector spaces

$$
0 \longrightarrow \mathscr{L} \longrightarrow \mathscr{V} \longrightarrow \mathscr{V} / \mathscr{L} \longrightarrow 0 .
$$

Then the group $P$ preserves this sequence. Therefore by Lemma 4.3, it is enough to prove that the homomorphisms $\mathscr{D e t}_{\mathscr{L}}$ and $\operatorname{Det}_{\mathscr{L} / V}$ are trivial on $P$. But this is obvious from the proof of Proposition 4.2.

For a colattice $\mathscr{L}^{c} \subset \mathcal{V}$ we have to use the analogous reasonings. 
4C. The two-dimensional story. If $\mathbb{V} \in \mathrm{Tate}_{2}$, then we denote by GL( $\left.\mathbb{V}\right)$ the group of automorphisms of $\mathbb{V}$ in this category.

There should be a determinantal functor from (Tate 2 , isom) to $B^{2} \mathscr{P i} c^{\mathbb{Z}}$, which assigns to every such $\mathbb{V}$ the graded gerbal theory in the sense of [Arkhipov and Kremnizer 2010], satisfying properties generalizing those listed in Proposition 3.8 (and further compatibility conditions). We do not make it precise. But we define the corresponding central extension of GL( $\mathbb{V})$ as follows. Pick a lattice $\mathbb{L}$ of $\mathbb{V}$. Then one associates with $g$ the $\mathscr{P} i c^{\mathbb{Z}}$-torsor

$$
\operatorname{Det}_{\mathbb{V}}(g)=\operatorname{Det}(\mathbb{L} \mid g \mathbb{L}):=\operatorname{Det}\left(\frac{g \mathbb{L}}{\mathbb{L} \cap g \mathbb{L}}\right)-\operatorname{Det}\left(\frac{\mathbb{L}}{\mathbb{L} \cap g \mathbb{L}}\right) \text {. }
$$

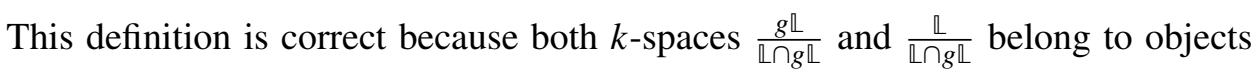
of category Tate $_{1}$. We define the 1-isomorphism as

$$
\operatorname{Det}\left(\mathbb{L} \mid g g^{\prime} \mathbb{L}\right) \simeq \operatorname{Det}(\mathbb{L} \mid g \mathbb{L})+\operatorname{Det}\left(g \mathbb{L} \mid g g^{\prime} \mathbb{L}\right) \simeq \operatorname{Det}(\mathbb{L} \mid g \mathbb{L})+\operatorname{Det}\left(\mathbb{L} \mid g^{\prime} \mathbb{L}\right) \text {. }
$$

One uses Proposition 3.8 to check that this defines a central extension of GL( $\mathbb{V})$ by $\mathscr{P} i c^{\mathbb{Z}}$. This central extension depends on the chosen lattice $\mathbb{L}$ of $\mathbb{V}$. If we change the lattice, then the central extension constructed by a new lattice will be isomorphic to the previous one.

Remark 4.8. If one replaces $\mathscr{P} i c^{\mathbb{Z}}$ by $\mathscr{P} i c$, such a central extension was constructed in [Osipov 2003; Frenkel and Zhu 2008]. In the first of these references the twodimensional tame symbol up to sign was obtained as an application of this construction, and the reciprocity laws on algebraic surfaces were proved up to sign.

As generalization of Lemma 4.3 and Lemma 4.7 it is not difficult to prove the following lemmas.

Lemma 4.9. If $0 \rightarrow \mathbb{V}^{\prime} \rightarrow \mathbb{V} \rightarrow \mathbb{V}^{\prime \prime} \rightarrow 0$ is a short exact sequence of 2-Tate vector spaces (recall that Tate $_{2}$ is an exact category). Let $P$ be the subgroup of GL(V) that preserves this sequence, then there is a canonical 1-isomorphism Det $\mathbb{V}^{\prime}+$ Det $_{\mathbb{V}^{\prime \prime}} \simeq$ Det $\sqrt{ }$ in $H^{2}\left(B P, \mathscr{P i} c^{\mathbb{Z}}\right)$.

Lemma 4.10. Let $P$ be subgroup of GL( $(\mathbb{V})$ which preserves a lattice or a colattice in $\mathbb{V}$, then the central extension restricted to $P$ can be trivialized.

Let $k^{\prime} / k$ be a finite field extension, and $\mathbb{K}=k^{\prime}((t))((s))$ be a two-dimensional local field. Then $\mathbb{K}$ has a natural structure as a 2-Tate vector space over $k$. The group $H=\mathbb{K}^{\times}$acts on $\mathbb{K}$ by left multiplications, which gives rise to an embedding $H \rightarrow \mathrm{GL}(\mathbb{K})$.

Theorem 4.11. For $f, g, h \in H$, one has

$$
C_{3}^{\mathscr{D} e t}(f, g, h)=\mathrm{Nm}_{k^{\prime} / k}\{f, g, h\},
$$


where the map $C_{3}^{\text {Det }}$ is constructed in Proposition 2.17 and $\{\cdot, \cdot, \cdot\}$ is the twodimensional tame symbol.

In what follows, we will denote the bimultiplicative homomorphism $C_{2}^{\text {Det }}$ by $C_{2}$, the homomorphism $C_{g}^{\mathscr{D} e t}$ by $C_{g}$ and the map $C_{3}^{\mathscr{D} e t}$ by $C_{3}$.

Proof. Since both maps $C_{3}$ and $\operatorname{Nm}_{k^{\prime} / k}\{\cdot, \cdot, \cdot\}$ are antisymmetric and trimultiplicative, we just need to consider the following cases: (i) $f, g, h \in \mathbb{O}_{\mathbb{K}}^{\times}$; (ii) $f, g \in$ $\mathcal{O}_{\mathbb{K}}^{\times}, h=s$; (iii) $f \in \mathbb{O}_{\mathbb{K}}^{\times}, g=h=s$; (iv) $f=g=h=s$. Here $\mathbb{O}_{\mathbb{K}}=k^{\prime}((t)) \llbracket s \rrbracket$ is the ring of integers of the field $\mathbb{K}$, which is also a lattice in $\mathbb{K}$. We will fix $\mathbb{L}=O_{\mathbb{K}}$.

In Case (i), both $C_{3}$ and $\operatorname{Nm}_{k^{\prime} / k}\{\cdot, \cdot, \cdot\}$ are trivial (to see that $C_{3}$ is trivial, one uses Lemma 4.10).

Case (ii). According to formulas (4-2)-(4-4), this case amounts to proving that

$$
C_{3}(f, g, s)=\operatorname{Nm}_{k^{\prime} / k}\{\bar{f}, \bar{g}\},
$$

where $\bar{f}, \bar{g}$ are the image of elements $f, g$ under the map $\mathbb{O}_{\mathbb{K}}^{\times} \rightarrow K^{\times}$.

Let us consider a little more general situation. Let $f, g \in \mathrm{GL}(\mathbb{K})$ that leave the lattice $O_{\mathbb{K}}$ invariant, and let $h \in \mathrm{GL}(\mathbb{K})$ such that $h O_{\mathbb{K}} \subset \mathrm{O}_{\mathbb{K}}$. Let $\mathscr{V}=\mathrm{O}_{\mathbb{K}} / h \mathrm{O}_{\mathbb{K}}$, which is a 1-Tate vector space over the field $k$. We assume that $f, g, h$ mutually commute with each other. Then $f, g: \mathbb{O}_{\mathbb{K}} \rightarrow \mathbb{O}_{\mathbb{K}}$ induce automorphisms $\pi_{h}(f), \pi_{h}(g): \mathscr{V} \rightarrow \mathscr{V}$. Let $\mathscr{D} e t$ be the central extension of GL(KK) by $\mathscr{P} i c^{\mathbb{Z}}$ defined by the lattice $\mathbb{L}=O_{\mathbb{K}}$. By definition, under the isomorphism

$$
\mathscr{L}: \mathscr{P i c} c^{\mathbb{Z}} \rightarrow \operatorname{Hom}_{\mathscr{P} i c^{\mathbb{Z}}}\left(\mathscr{D e t}\left(\bigcirc_{\mathbb{K}} \mid h g \bigcirc_{\mathbb{K}}\right), \operatorname{Det}\left(\bigcirc_{\mathbb{K}} \mid h g \mathcal{O}_{\mathbb{K}}\right)\right) \text {, }
$$

the 1-isomorphism $C_{2}(h, g)$ corresponds to the composition of 1-isomorphisms of $\mathscr{P} i c^{\mathbb{Z}}$-torsors:

$$
\begin{aligned}
& \operatorname{Det}\left(\mathcal{O}_{\mathbb{K}} \mid h g \mathcal{O}_{\mathbb{K}}\right) \rightarrow \operatorname{Det}\left(\mathcal{O}_{\mathbb{K}} \mid h O_{\mathbb{K}}\right)+\operatorname{Det}\left(h \mathscr{O}_{\mathbb{K}} \mid h g \mathscr{O}_{\mathbb{K}}\right) \\
& \rightarrow \operatorname{Det}\left(\mathrm{O}_{\mathbb{K}} \mid h O_{\mathbb{K}}\right)+\operatorname{Det}\left(O_{\mathbb{K}} \mid g O_{\mathbb{K}}\right) \\
& \rightarrow \operatorname{Det}\left(\mathrm{O}_{\mathbb{K}} \mid g \mathrm{O}_{\mathbb{K}}\right)+\operatorname{Det}\left(\mathscr{O}_{\mathbb{K}} \mid h \mathrm{O}_{\mathbb{K}}\right) \\
& \rightarrow \operatorname{Det}\left(\mathcal{O}_{\mathbb{K}} \mid g \mathcal{O}_{\mathbb{K}}\right)+\operatorname{Det}\left(g \mathcal{O}_{\mathbb{K}} \mid g h \mathcal{O}_{\mathbb{K}}\right) \rightarrow \operatorname{Det}\left(\mathcal{O}_{\mathbb{K}} \mid g h \mathcal{O}_{\mathbb{K}}\right) \text {. }
\end{aligned}
$$

Using the fact that $g \mathscr{O}_{\mathbb{K}}=\mathscr{O}_{\mathbb{K}}$ and Proposition 3.8, this 1-isomorphism is canonically 2-isomorphic to the 1-isomorphism

$$
\operatorname{Det}\left(\mathcal{O}_{\mathbb{K}} \mid h g \mathcal{O}_{\mathbb{K}}\right) \stackrel{\mathscr{L}(\mathscr{D e t V}(\pi(g)))}{\longrightarrow} \operatorname{Det}\left(\mathcal{O}_{\mathbb{K}} \mid h g \mathcal{O}_{\mathbb{K}}\right)
$$

Therefore, there is a canonical 2-isomorphism $C_{2}(h, g) \simeq-\operatorname{Det}_{V}\left(\pi_{h}(g)\right)$, because, by definition (see formula (4-6)), $\operatorname{Det}\left(\mathcal{O}_{\mathbb{K}} \mid h \mathcal{O}_{\mathbb{K}}\right) \simeq-\operatorname{Det}(\mathscr{V})$. One readily checks by the construction of Lemma-Definition 2.13, that these 2-isomorphisms fit into 
the commutative diagrams

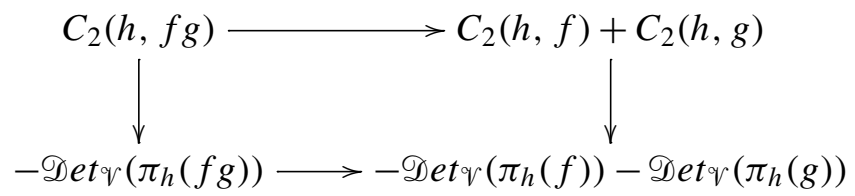

where the natural isomorphism $\operatorname{Det}_{\mathscr{V}}\left(\pi_{h}(f g)\right) \rightarrow \operatorname{Det}_{\mathscr{V}}\left(\pi_{h}(f)\right)+\operatorname{Det}_{\mathscr{V}}\left(\pi_{h}(g)\right)$ comes from Proposition 4.2. (We have to use that Det $((0))$ is canonically isomorphic to $\mathscr{P} i c^{\mathbb{Z}}$, and $\mathcal{O}_{\mathbb{K}} / g \mathcal{O}_{\mathbb{K}}=(0)$, where (0) is the zero-space.)

We now return to our proof of case (ii). Let $P_{s}$ be the subgroup of GL( $\left.\mathbb{K}\right)$ consisting of elements that preserve the lattice $O_{\mathbb{K}}$ and commute with the element $s$. Then the elements in the group $P_{s}$ also preserve the lattice $s \mathcal{O}_{\mathbb{K}}$, and therefore induce a group homomorphism

$$
\pi_{s}: P_{s} \rightarrow \mathrm{GL}(K)
$$

because $\mathbb{K}=\mathscr{O}_{\mathbb{K}} / s \mathcal{O}_{\mathbb{K}}$. Then the commutative diagram (4-8) amounts to the following lemma.

Lemma 4.12. The homomorphism $C_{s}: P_{s} \rightarrow \mathscr{P i c} c^{\mathbb{Z}}$ is isomorphic to the minus (or the inverse) of the homomorphism

$$
\operatorname{Det}_{K} \circ \pi_{s}: P_{s} \rightarrow \mathrm{GL}(K) \rightarrow \mathscr{P i c}{ }^{\mathbb{Z}} .
$$

By Proposition 4.5, we thus obtain that

$$
C_{3}(f, g, s)=C_{3}(s, f, g)=\operatorname{Comm}\left(C_{s}\right)(f, g)=\operatorname{Nm}_{k^{\prime} / k}\{\bar{f}, \bar{g}\}
$$

for $f, g \in \mathbb{O}_{\mathbb{K}}^{\times} \subset \mathrm{GL}(\mathbb{K})$. Case (ii) follows.

Case (iii). According to formulas (4-2)-(4-4), one needs to show

$$
C_{3}(f, s, s)=C_{f}(s, s)=\operatorname{Nm}_{k^{\prime} / k}(-1)^{\nu_{2}(\bar{f})}=(-1)^{\left(\nu_{2}(\bar{f})\left[k^{\prime}: k\right]\right)}=(-1)^{\left(\nu_{2}(\bar{f})\left[k^{\prime}: k\right]\right)^{2}} .
$$

We have the following exact sequence of 1-Tate vector spaces

$$
0 \longrightarrow \frac{s \mathrm{O}_{\mathbb{K}}}{s^{2} \mathrm{O}_{\mathbb{K}}} \rightarrow \frac{\mathrm{O}_{\mathbb{K}}}{s^{2} \mathrm{O}_{\mathbb{K}}} \rightarrow \frac{\mathrm{O}_{\mathbb{K}}}{s \mathrm{O}_{\mathbb{K}}} \longrightarrow 0 .
$$

and therefore by Lemma 4.3, for any element $p \in P_{s}$, there is a canonical isomorphism in $\mathscr{P i c} c^{\mathbb{Z}}$

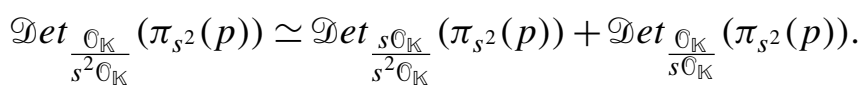


On the other hand, we have already shown that there are canonical isomorphisms

$$
\begin{aligned}
C_{2}(s, p) & \simeq-\operatorname{Det}_{\frac{\mathscr{O}_{K}}{s O_{K}}}\left(\pi_{s}(p)\right)=-\operatorname{Det}_{\frac{\mathscr{O}_{K}}{s O_{K}}}\left(\pi_{s^{2}}(p)\right), \\
C_{2}\left(s^{2}, p\right) & \simeq-\operatorname{Det}_{\frac{\mathscr{O}_{K}}{s^{2} \mathscr{O}_{K}}}\left(\pi_{s^{2}}(g)\right) .
\end{aligned}
$$

Again, by checking the construction as in Lemma-Definition 2.13, one obtains that under the isomorphisms (4-10), the canonical isomorphism

$$
C_{2}\left(s^{2}, p\right) \simeq C_{2}(s, p)+C_{2}(s, p)
$$

corresponds to (4-9).

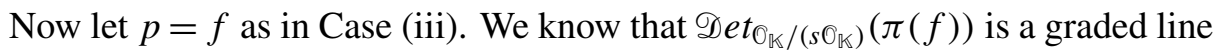
of degree $v_{2}(\bar{f})\left[k^{\prime}: k\right]$. Therefore, using $C_{2}(a, b) \simeq-C_{2}(b, a)$ for any commuting elements $a, b \in \mathrm{GL}(\mathbb{K})$, we obtain that Case (iii) follows from the definition of the commutativity constraints in $\mathscr{P} i c^{\mathbb{Z}}$.

Case (iv). One needs to show that $C_{s}(s, s)=1$. One can easily show that there are canonical isomorphisms $C_{2}(s, s) \simeq \ell_{0}, C_{2}\left(s^{2}, s\right) \simeq \ell_{0}$, and the canonical isomorphism $C_{2}\left(s^{2}, s\right) \simeq C_{2}(s, s)+C_{2}(s, s)$ corresponds to $\ell_{0} \simeq \ell_{0}+\ell_{0}$. (We used that for the $k^{\prime}$-space $M=k^{\prime} \llbracket t \rrbracket((s))$ we have $s M=M$, and the $k^{\prime}$-space $M$ induce a lattice in every 1 -Tate vector space $s^{n} \mathbb{O}_{\mathbb{K}} / s^{n+l} \mathrm{O}_{\mathbb{K}}, n \in \mathbb{Z}, l \in \mathbb{N}$.) This case also follows.

\section{Reciprocity laws}

We will use the adèle theory on schemes. Adèles on algebraic surfaces were introduced in [Parshin 1976]. On arbitrary noetherian schemes they were considered in [Beilinson 1980]. See the proof of part of results of this latter reference in [Huber 1991]. A survey of adèles can be found in [Osipov 2008].

We fix a perfect field $k$.

5A. Weil reciprocity law. To fix the idea, let us first revisit the Weil reciprocity law. Let $C$ be an irreducible projective curve over a field $k$. Let $k(C)$ be the field of rational functions on the curve $C$. For a closed point $p \in C$ let $\hat{O}_{p}$ be the completion by maximal ideal $m_{p}$ of the local ring $0_{p}$ of point $p \in C$. Let a ring $K_{p}$ be the localization of the ring $\hat{O}_{p}$ with respect to the multiplicative system $\hat{O}_{p} \backslash 0$. (If $p$ is a smooth point, then $K_{p}=k(C)_{p}$ is the fraction field of the ring $\hat{\mathrm{O}}_{p}$, and $K_{p}=k(p)\left(\left(t_{p}\right)\right), \hat{\mathrm{O}}_{p}=k(p) \llbracket t_{p} \rrbracket$, where $k(p)$ is the residue field of the point $p$, $t_{p}$ is a local parameter at $p$. For a nonsmooth point $p \in C$, the ring $K_{p}$ is a finite direct product of one-dimensional local fields.)

We have that $K_{p}$ is a 1 -Tate vector space over $k$, and $\hat{O}_{p}$ is a lattice in $K_{p}$ for any point $p \in C$. 
For any coherent subsheaf $\mathscr{F}$ of the constant sheaf $k(C)$ on the curve $C$ we consider the following adèle complex $\mathscr{A}_{C}(\mathscr{F})$ :

$$
\mathbb{A}_{C, 0}(\mathscr{F}) \oplus \mathbb{A}_{C, 1}(\mathscr{F}) \longrightarrow \mathbb{A}_{C, 01}(\mathscr{F})
$$

whose cohomology groups coincide with the cohomology groups $H^{*}(C, \mathscr{F})$. We recall that

$$
\begin{gathered}
\mathbb{A}_{C, 0}(\mathscr{F})=k(C) \otimes_{\mathscr{O}_{C}} \mathscr{F}_{\mathcal{F}}, \quad \mathbb{A}_{C, 1}(\mathscr{F})=\prod_{p \in C} \hat{\mathscr{O}}_{p} \otimes_{\mathscr{O}_{C}} \mathscr{F}, \\
\mathbb{A}_{C, 01}(\mathscr{F})=\mathbb{A}_{C}=\prod_{p \in C}^{\prime} K_{p} \otimes_{\mathscr{O}_{C}} \mathscr{F}_{\mathscr{F}}
\end{gathered}
$$

where $\prod^{\prime}$ denotes the restricted (adèle) product with respect to $\prod_{p \in C} \hat{\widehat{O}}_{p}$. Observe that since $\mathscr{F}$ is a subsheaf of $k(C)$, we have

$$
k(C) \otimes_{\mathscr{O}_{C}} \mathscr{F}=k(C), \quad K_{p} \otimes_{\mathscr{O}_{C}} \mathscr{F}_{F}=K_{p} .
$$

The adèle ring $\mathbb{A}_{C}$ is a 1-Tate vector space over $k$. This is because

$$
\mathbb{A}_{C}=\lim _{\mathscr{G} \subset k(C)} \underset{\mathscr{H} \subset \bar{C}(C)}{\lim _{C, 1}} \mathbb{A}_{C}(\mathscr{H}) / \mathbb{A}_{C, 1}(\mathscr{G})
$$

and $\operatorname{dim}_{k} \mathbb{A}_{C, 1}(\mathscr{H}) / \mathbb{A}_{C, 1}(\mathscr{G})<\infty$ for coherent subsheaves $0 \neq \mathscr{G} \subset \mathscr{H}$ of $k(C)$. (We used that $\mathbb{A}_{C, 1}(\mathscr{H}) / \mathbb{A}_{C, 1}(\mathscr{G})=\mathbb{A}_{C, 1}=\bigoplus_{p \in C} \hat{0}_{p} \otimes_{\mathscr{O}_{C}}(\mathscr{H} / \mathscr{G})$ ). For any coherent subsheaf $\mathscr{F}_{C}$ of $k(C)$ the space $\mathbb{A}_{C, 1}\left(\mathscr{F}_{F}\right)$ is a lattice in the space $\mathbb{A}_{C}$. Hence, the $k$-space $k(C)$ is a colattice in $\mathbb{A}_{C}$, since from the adelic complex $\mathscr{A}(\mathscr{F})$ it follows that

$$
\begin{gathered}
\operatorname{dim}_{k} k(C) \cap \mathbb{A}_{C, 1}(\mathscr{F})=\operatorname{dim}_{k} H^{0}(C, \mathscr{F})<\infty, \\
\operatorname{dim}_{k} \mathbb{A}_{C} /\left(k(C)+\mathbb{A}_{C, 1}(\mathscr{F})\right)=\operatorname{dim}_{k} H^{1}(C, \mathscr{F})<\infty .
\end{gathered}
$$

Let a $p$ be a point of $C$ and $f, g$ a pair of elements of $K_{p}^{\times}$. If $K_{p}=k(p)\left(\left(t_{p}\right)\right)$, then we denote by $\{f, g\}_{p}$ the element from $k(p)^{\times}$which is the corresponding tame symbol. If the ring $K_{p}$ is isomorphic to the finite product of fields isomorphic to $k(p)((t))$, then we denote by $\{f, g\}_{p}$ the element from $k(p)^{\times}$which is the same finite product of the corresponding tame symbols. Recall that there is the diagonal embedding $k(C) \hookrightarrow \mathbb{A}_{C}$.

Proposition 5.1 (Weil reciprocity law). For any elements $f, g \in k(C)^{\times}$the following product contains only finitely many nonequal to 1 terms and

$$
\prod_{p \in C} \mathrm{Nm}_{k(p) / k}\{f, g\}_{p}=1 .
$$

Proof. By Proposition 4.5, we can change $\mathrm{Nm}_{k(p) / k}\{f, g\}_{p}$ to $\operatorname{Comm}\left(\mathscr{D e t}_{K_{p}}\right)(f, g)$ for all $p \in C$ in (5-1). There are points $p_{1}, \ldots, p_{l} \in C$ such that if $p \in C$ and 
$p \neq p_{i}(1 \leq i \leq l)$, then $f \hat{\mathscr{O}}_{p}=\hat{\mathrm{O}}_{p}, g \hat{\mathscr{O}}_{p}=\hat{\mathrm{O}}_{p}$, and hence, by Lemma 4.7, $\operatorname{Comm}\left(\mathscr{D e t}_{K_{p}}\right)(f, g)=1$ for points $p \neq p_{i}(1 \leq i \leq l)$.

We define the group $H$ as the subgroup of the group $k(C)^{\times}$generated by the elements $f$ and $g$. We apply Lemma 4.3 to the 1 -Tate $k$-vector spaces

$$
\mathscr{V}=\mathbb{A}_{C}, \quad \mathscr{V}^{\prime}=\mathbb{A}_{C \backslash\left\{p_{1}, \ldots, p_{l}\right\}}, \quad \mathscr{V}^{\prime \prime}=\prod_{1 \leq i \leq l} K_{p_{i}} .
$$

The group $H$ preserves the lattice $\prod_{p \in C \backslash\left\{p_{1}, \ldots, p_{l}\right\}} \hat{\widehat{O}}_{p}$ in the space $\mathscr{V}^{\prime}$. Therefore, by Lemma 4.7, the homomorphism Det $_{\mathbb{A}_{C}}$ is isomorphic to the homomorphism Det ${ }^{\prime \prime}$, which is (again by Lemma 4.3) isomorphic to the sum of homomorphisms Det $_{K_{p_{1}}}, \ldots$, Det $K_{p_{l}}$. Since the group $H$ preserves the colattice $k(C)$ in $\mathbb{A}_{C}$, the homomorphism $\mathscr{D e t}_{\mathbb{A}_{C}}$ is isomorphic to the trivial one (by Lemma 4.7). Now using Remark 2.7 and Corollaries 2.9 and 2.10 we obtain (5-1).

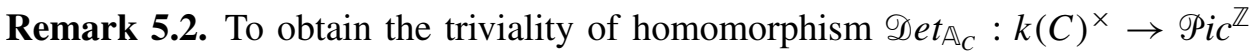
in an explicit way, one has to use the following canonical isomorphism for any $g \in k(C)^{\times}$:

$$
\operatorname{Det}_{\mathbb{A}_{C}}(g) \simeq \operatorname{det}\left(H^{*}\left(\mathscr{A}_{C}\left(g \mathscr{O}_{C}\right)\right)\right) \otimes \operatorname{det}\left(H^{*}\left(\mathscr{A}_{C}\left(\mathscr{O}_{C}\right)\right)\right)^{-1},
$$

where for any coherent sheaf $\mathscr{F}$ on $C$

$$
\begin{aligned}
\operatorname{det}\left(H^{*}\left(\mathscr{A}_{C}(\mathscr{F})\right)\right):=\operatorname{det}\left(H^{0}\left(\mathscr{A}_{C}(\mathscr{F})\right)\right) \otimes \operatorname{det}\left(H^{1}\left(\mathscr{A}_{C}(\mathscr{F})\right)\right)^{-1} & \\
& \simeq \operatorname{det}\left(H^{0}(C, \mathscr{F})\right) \otimes \operatorname{det}\left(H^{1}(C, \mathscr{F})\right)^{-1} .
\end{aligned}
$$

(Formula (5-2) easily follows from adèle complexes and (4-5) if we change in (4-5) the lattices $\mathscr{L}$ and $g \mathscr{L}$ in $\mathbb{A}_{C}$ to any two lattices coming from nonzero coherent subsheaves $\mathscr{G} \subset \mathscr{H}$ of $k(C)$, and change correspondingly in formula (5-2) the sheaves $\mathcal{O}$ and $g \mathcal{O}$ to the sheaves $\mathscr{G} \subset \mathscr{H}$.) Now the homomorphism $\mathscr{D}_{e t_{\mathbb{A}_{C}}}$ is isomorphic to the trivial one by formula (5-2) and the fact that multiplication on an element $g \in k(C)^{*}$ gives a canonical isomorphism between adèle complexes $\mathscr{A}_{C}\left(\mathscr{O}_{C}\right)$ and $\mathscr{A}_{C}\left(g \mathscr{O}_{C}\right)$, which induce the canonical isomorphism between $\operatorname{det}\left(H^{*}\left(\mathscr{A}_{C}\left(\mathscr{O}_{C}\right)\right)\right)$ and $\operatorname{det}\left(H^{*}\left(\mathscr{A}_{C}\left(g \mathscr{O}_{C}\right)\right)\right)$.

5B. Parshin reciprocity laws. Let $X$ be an algebraic surface over the field $k$. We assume, for simplicity, that $X$ is a smooth connected surface.

We consider pairs $x \in C$, where $C$ are irreducible curves on $X$ and $x$ are closed points on $C$. For every such pair one can define the ring $K_{x, C}$, which will be a finite product of two-dimensional local fields, as follows. Assume that the curve $C$ on $X$ has the formal branches $\mathbf{C}_{1}, \ldots, \mathbf{C}_{n}$ at the point $x \in C$; that is,

$$
\left.C\right|_{\operatorname{Spec} \hat{O}_{x}}=\bigcup_{1 \leq i \leq n} \mathbf{C}_{i}
$$


where $\hat{\mathscr{O}}_{x}$ is the completion of the local ring $\mathcal{O}_{x}$ of a point $x \in X$, and $\mathbf{C}_{i}$ is irreducible in Spec $\hat{O}_{x}$ for any $1 \leq i \leq n$. (Since we assumed $X$ is smooth, $\hat{\mathrm{O}}_{x} \simeq k(x) \llbracket t_{1}, t_{2} \rrbracket$.) Now every $\mathbf{C}_{i}$ defines a discrete valuation on the fraction field Frac $\hat{O}_{x}$. We define a two-dimensional local field $K_{x, \mathbf{C}_{i}}$ as the completion of the field Frac $\hat{O}_{x}$ with respect to this discrete valuation, and let $\hat{O}_{x}, \mathbf{C}_{i}$ be the valuation ring. Then we define

$$
K_{x, C}:=\bigoplus_{1 \leq i \leq n} K_{x, \mathbf{C}_{i}}, \quad \hat{\mathrm{O}}_{x, C}:=\bigoplus_{1 \leq i \leq n} \hat{\mathrm{O}}_{x, \mathbf{C}_{i}},
$$

Observe that if $x \in C$ is a smooth point, then $\hat{O}_{x, C} \simeq k(x)((t)) \llbracket s \rrbracket$ and $K_{x, C} \simeq$ $k(x)((t))((s))$. It is clear that the ring $\hat{O}_{x}$ diagonally embeds into the ring $K_{x, C}$.

Let us also define $B_{x} \subset K_{x, C}$ as $\underline{\lim }_{n>0} s_{C}^{-n} \hat{\widehat{O}}_{x}$, where a local equation $s_{C}=0$ determines $C$ on some open $X \supset V \vec{\ni} x$. It is clear that the subring $B_{x}$ of $K_{x, C}$ does not depend on the choice of such $s_{C}$ when $V \ni x$. If $x \in C$ is a smooth point, and $K_{x, C}=k(x)((t))\left(\left(s_{C}\right)\right)$, where $s_{C}=0$ is a local equation of the curve $C$ on $X$ near the point $x$ and $t=0$ defines a transversal curve locally on $X$ near $x$, then $B_{x}=k(x) \llbracket t \rrbracket\left(\left(s_{C}\right)\right)$.

Any ring $K_{x, C}$ is a 2-Tate vector space over $k(x)$ (and therefore over $k$ ), and the ring $\hat{\mathrm{O}}_{x, C}$ is a lattice in $K_{x, C}$.

Let

$$
f=\bigoplus_{1 \leq i \leq n} f_{i}, \quad g=\bigoplus_{1 \leq i \leq n} g_{i}, \quad h=\bigoplus_{1 \leq i \leq n} h_{i} .
$$

be elements of $K_{x, C}^{\times}$. We define an element of $k(x)^{\times}$by

$$
\{f, g, h\}_{x, C}:=\prod_{1 \leq i \leq n}\left\{f_{i}, g_{i}, h_{i}\right\}_{x}, \mathbf{C}_{i},
$$

where $\left\{f_{i}, g_{i}, h_{i}\right\}_{x}, \mathbf{C}_{i}$ is the two-dimensional tame symbol associated to the twodimensional local field $K_{x, \mathbf{C}_{i}}$ (cf. Section 4A).

Fix a point $x \in X$. For any free finitely generated $\hat{O}_{x}$-module subsheaf $\mathscr{F}_{F}$ of the constant sheaf Frac $\hat{O}_{x}$ on the scheme Spec $\hat{O}_{x}$ we consider the following adèle complex $\mathscr{A}_{X, x}(\mathscr{F})$ :

$$
\mathbb{A}_{X, x, 0}(\mathscr{F}) \oplus \mathbb{A}_{X, x, 1}(\mathscr{F}) \longrightarrow \mathbb{A}_{X, x, 01}(\mathscr{F}) .
$$

This is the adèle complex on the one-dimensional scheme $U_{x}:=\operatorname{Spec} \hat{\mathscr{O}}_{x} \backslash x$ for the sheaf $\mathscr{F}_{U_{x}}$, and, hence, the cohomology groups of this complex coincide with the cohomology groups $H^{*}\left(U_{x},\left.\mathscr{F}\right|_{U_{x}}\right)$. By definition, we have

$$
\mathbb{A}_{X, x, 0}(\mathscr{F})=\operatorname{Frac} \hat{O}_{x}, \quad \mathbb{A}_{X, x, 1}(\mathscr{F})=\prod_{\mathbf{C} \ni x} \hat{O}_{x, \mathbf{C}} \otimes_{\hat{O}_{x}} \mathscr{F}, \quad \mathbb{A}_{X, x, 01}(\mathscr{F})=\mathbb{A}_{X, x}=\prod_{\mathbf{C} \ni x}^{\prime} K_{p, \mathbf{C}},
$$


where the product is taken over all prime ideals $\mathbf{C}$ of height 1 of the ring $\hat{\mathrm{O}}_{x}$, and $\prod^{\prime}$ denotes the restricted (adèle) product with respect to $\prod_{\mathbf{C} \ni x} \hat{0}_{x}, \mathbf{C}$.

Observe that the adèle ring $\mathbb{A}_{X, x}$ is a 2-Tate vector space over the field $k(x)$. This is because

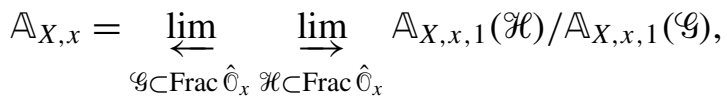

and $\mathbb{A}_{X, x, 1}(\mathscr{H}) / \mathbb{A}_{X, x, 1}(\mathscr{G})$ is a 1-Tate vector space for free $\hat{0}_{x}$-module subsheaves $0 \neq \mathscr{G} \subset \mathcal{H}$ of Frac $\hat{O}_{x}$. (We used that $\mathbb{A}_{X, x, 1}(\mathcal{H}) / \mathbb{A}_{X, x, 1}(\mathscr{G})=\bigoplus_{\mathbf{C} \ni x} \hat{\hat{O}}_{x, \mathbf{C}} \otimes_{\hat{O}_{x}}$ $\mathscr{H} / \mathscr{G}_{\text {. }}$ ) For any free finitely generated $\hat{O}_{x}$-module subsheaf $\mathscr{F}_{F}$ of Frac $\hat{O}_{x}$ the space $\mathbb{A}_{X, x, 1}(\mathscr{F})$ is a lattice in the space $\mathbb{A}_{X, x}$.

From Proposition 8 in [Osipov 2005] it follows that the $k(x)$-vector spaces $H^{0}\left(\mathscr{A}_{X, x}(\mathscr{F})\right)$ and $H^{1}\left(\mathscr{A}_{X, x}(\mathscr{F})\right)$ are 1-Tate vector spaces. Indeed, since $x$ is a smooth point of $X$,

$$
H^{0}\left(\mathscr{A}_{X, x}(\mathscr{F})\right)=H^{0}\left(U_{x},\left.\mathscr{F}\right|_{U_{x}}\right)=\mathscr{F}
$$

is a projective limit of finite-dimensional $k(x)$-vector spaces $\mathscr{F} / m_{x}^{n} \mathscr{F}\left(m_{x}\right.$ is the maximal ideal of the ring $\hat{0}_{x}$ ), and

$$
H^{1}\left(\mathscr{A}_{X, x}(\mathscr{F})\right)=H^{1}\left(U_{x}, \mathscr{F}_{U_{x}}\right)=\underset{n>0}{\lim _{n \rightarrow 0}} \operatorname{Ext}_{\hat{\mathrm{O}}_{x}}^{2}\left(\hat{\mathrm{O}}_{x} / m_{x}^{n}, \mathscr{F}\right),
$$

where for any $n>0$ the space $\operatorname{Ext}_{\hat{O}_{x}}^{2}\left(\hat{\mathscr{O}}_{x} / m_{x}^{n}, \mathscr{F}\right)$ is a finite-dimensional over the field $k(x)$ vector space; see, for example, [Osipov 2005, Lemma 6].

Fix an irreducible projective curve $C$ on $X$. For any invertible $0_{X}$-subsheaf $\mathscr{F}$ of the constant sheaf $k(X)$ on $X$ we consider the following adèle complex $\mathscr{A}_{X, C}(\mathscr{F})$

$$
\mathbb{A}_{X, C, 0}(\mathscr{F}) \oplus \mathbb{A}_{X, C, 1}(\mathscr{F}) \longrightarrow \mathbb{A}_{X, C, 01}(\mathscr{F}) .
$$

where $\mathbb{A}_{X, C, 0}(\mathscr{F}):=K_{C}, \mathbb{A}_{X, C, 01}(\mathscr{F}):=\mathbb{A}_{X, C}=\mathbb{A}_{C}\left(\left(s_{C}\right)\right)$, and

$$
\mathbb{A}_{X, C, 1}(\mathscr{F}):=\left(\prod_{x \in C} B_{x} \otimes_{\mathscr{O}_{X}} \mathscr{F}\right) \cap \mathbb{A}_{X, C} .
$$

Here $K_{C}$ is the completion of the field $k(X)$ with respect to the discrete valuation given by the curve $C$ on $X$. (If $s_{C}=0$ is a local equation of the curve $C$ on some open subset $V$ of $X$ such that $V \cap C \neq \varnothing$, then $K_{C}=k(C)\left(\left(s_{C}\right)\right)$.) The ring $\mathbb{A}_{X, C}$ is a subring of $\prod_{x \in C} K_{x, C}$, and does not depend on the choice of $s_{C}$. The intersection (5-4) is taken in the ring $\prod_{x \in C} K_{x, C}$. 
We note that from [Osipov 2005, § 5.1] it follows that the complex $\mathscr{A}_{X, C}(\mathscr{F})$ coincides with the following complex

$$
\underset{n}{\lim } \lim _{m>n} \mathscr{A}_{\left(C, \bigcirc_{X} / J_{C}^{m-n}\right)}\left(\mathscr{F} \otimes_{\mathscr{O}_{X}} J_{C}^{n} / J_{C}^{m}\right)
$$

Here $J_{C}$ is the ideal sheaf of the curve $C$ on $X,\left(C, O_{X} / J_{C}^{m-n}\right)$ is a one-dimensional scheme which has the topological space $C$ and the structure sheaf $\mathscr{O}_{X} / J_{C}^{m-n}$, and $A_{\left(C, O_{X} / J_{C}^{m-n}\right)}\left(\mathscr{F}_{F} \otimes_{\mathcal{O}_{X}} J_{C}^{n} / J_{C}^{m}\right)$ is the adèle complex of the coherent sheaf $\mathscr{F}_{\mathcal{F}} \otimes_{\mathcal{O}_{X}}$ $J_{C}^{n} / J_{C}^{m}$ on the scheme $\left(C, O_{X} / J_{C}^{m-n}\right)$. Hence and from the proof of [Osipov 2005, Proposition 12] we obtain that

$$
H^{*}\left(\mathscr{A}_{X, C}(\mathscr{F})\right)=\underset{n}{\lim } \lim _{m>n} H^{*}\left(C, \mathscr{F} \otimes_{\mathcal{O}_{X}} J_{C}^{n} / J_{C}^{m}\right)
$$

where for $i=0$ and $i=1$ we have $\operatorname{dim}_{k} H^{i}\left(C, \mathscr{F}_{\mathcal{F}} \otimes_{\mathfrak{O}_{X}} J_{C}^{n} / J_{C}^{m}\right)<\infty$. For $i=0$ and $i=1$ the $k$-vector space $H^{i}\left(\mathscr{A}_{X, C}(\mathscr{F})\right)$ has the natural topology of inductive and projective limits. It is not difficult to see that the space $H^{0}\left(\mathscr{A}_{X, C}(\mathscr{F})\right)$ is a locally linearly compact $k$-vector space; i.e., it is a 1-Tate vector space. But the space $H^{1}\left(\mathscr{A}_{X, C}(\mathscr{F})\right)$ is not a Hausdorff space in this topology. Let $\tilde{H}^{1}\left(\mathscr{A}_{X, C}(\mathscr{F})\right)$ be the quotient space of $H^{1}\left(\mathscr{A}_{X, C}(\mathscr{F})\right)$ by the closure of zero. Then the space $\tilde{H}^{1}\left(\mathscr{A}_{X, C}(\mathscr{F})\right)$ is a locally linearly compact $k$-vector space, i.e., a 1 -Tate vector space.

We note that for any invertible subsheaves $0 \neq \mathscr{G} \subset \mathscr{H}$ of $k(X)$ we have that the space $B_{x} \otimes_{\hat{O}_{x}}(\mathscr{H} / \mathscr{G})$ is a 1 -Tate vector space, which is equal to zero for almost all points $x \in C$. Hence, we obtain that the space

$$
\mathbb{A}_{X, C, 1}(\mathscr{H}) / \mathbb{A}_{X, C, 1}(\mathscr{G})=\bigoplus_{x \in C} B_{x} \otimes_{\hat{O}_{x}}(\mathscr{H} / \mathscr{G})
$$

is a 1-Tate vector space.

For any point $x \in X$, we define a ring $K_{x}$ as the localization of the ring $\hat{\mathscr{O}}_{x}$ with respect to the multiplicative system $\hat{O}_{x} \backslash 0$. (We note that inside of the field Frac $\hat{O}_{x}$ the ring $K_{x}$ is defined as the product of two subrings: $\hat{O}_{x}$ and $k(X)$.)

For any pair $x \in C$ (where $C$ is an irreducible curve on $X$ and $x \in C$ is a closed point), we have the natural embeddings $k(X) \hookrightarrow K_{x}, k(X) \hookrightarrow K_{C}$ (recall that $K_{C}$ is the completion of the field $k(X)$ with respect to the discrete valuation given by the curve $C$ ). In addition, there are the natural embeddings $K_{x}, K_{C} \hookrightarrow K_{x, C}$. Therefore, we obtain

$$
k(X) \hookrightarrow K_{x} \hookrightarrow \mathbb{A}_{X, x}, \quad k(X) \hookrightarrow K_{C} \hookrightarrow \mathbb{A}_{X, C} .
$$

Theorem 5.3 (Parshin reciprocity laws). (1) Fix a point $x \in X$. Consider elements $f, g, h$ of the group $K_{x}^{\times}$of invertible elements of the ring $K_{x}$. Then the 
following product in $k(x)^{\times}$contains only finitely many terms distinct from 1 and

$$
\prod_{C \ni x}\{f, g, h\}_{x, C}=1 .
$$

(2) Fix a projective irreducible curve $C$ on $X$. Let elements $f, g, h$ be from the group $K_{C}^{\times}$. Then the following product in $k^{\times}$contains only finitely many terms distinct from 1 and

$$
\prod_{x \in C} \mathrm{Nm}_{k(x) / k}\{f, g, h\}_{x, C}=1 .
$$

Proof. We first prove formula (5-5). By Theorem 4.11, for any $f, g, h \in K_{x, \mathbf{C}}^{\times}$we have

$$
\{f, g, h\}_{x, \mathbf{C}}=C_{3}^{\mathscr{D} e t_{x, \mathbf{C}}}(f, g, h)
$$

for all prime ideals $\mathbf{C}$ of height 1 of the ring $\hat{O}_{x}$, where the central extension $\mathscr{D e t}_{x, \mathbf{C}}$ of the group $K_{x, \mathbf{C}}^{\times}$by the Picard groupoid $\mathscr{P} i c^{\mathbb{Z}}$ is constructed by formula (4-6) from the 2-Tate vector space $K_{x, \mathbf{C}}$ over the field $k(x)$ and the lattice $\hat{\mathcal{O}}_{x, \mathbf{C}}$ as in Section $4 \mathrm{C}$. We note that for almost all prime ideals $\mathbf{C}$ of height 1 of ring $\hat{\mathcal{O}}_{x}$, and for any elements $f, g, h$ from the group Frac $\hat{O}_{x}^{\times}$, we have $f \mathscr{O}_{x, \mathbf{C}}=\mathfrak{O}_{x}, \mathbf{C}, g \mathscr{O}_{x}, \mathbf{C}=\mathfrak{O}_{x}, \mathbf{C}$, and $h \mathbb{O}_{x, \mathbf{C}}=\mathfrak{O}_{x}, \mathbf{C}$. Then by Lemma 4.10 and Corollary 2.19, for almost all prime ideals $\mathbf{C}$ of height 1 of ring $\hat{O}_{x}$ we have $C_{3}^{\mathscr{D} e t_{x, \mathbf{C}}}(f, g, h)=1$.

We will prove that the central extension $\mathscr{D}_{e} t_{x}$ of Frac $\hat{O}_{x}^{\times}\left(\subset \operatorname{GL}\left(\mathbb{A}_{X, x}\right)\right)$ by $\mathscr{P} i c^{\mathbb{Z}}$ constructed by the 2-Tate vector space $\mathbb{A}_{X, x}$ and the lattice $\mathbb{A}_{X, x, 1}\left(\hat{\mathscr{O}}_{x}\right)$ using formula (4-6) can be trivialized in an explicit way. Observe that for any $d \in$ Frac $\hat{O}_{x}^{x}$, there is a canonical isomorphism of $\mathscr{P} i c^{\mathbb{Z}}$-torsors:

$$
\operatorname{Det}\left(\mathbb{A}_{X, x, 1}\left(\hat{\mathscr{O}}_{x}\right) \mid \mathbb{A}_{X, x, 1}\left(d \hat{\widehat{O}}_{x}\right)\right) \simeq \operatorname{Det}\left(H^{*}\left(\mathscr{A}_{X, x}\left(d \hat{\mathscr{O}}_{x}\right)\right)\right)-\operatorname{Det}\left(H^{*}\left(\mathscr{A}_{X, x}\left(\hat{\mathscr{O}}_{x}\right)\right)\right),
$$

where for any free subsheaf $\mathscr{F}$ of Frac $\hat{O}_{x}$ on the scheme Spec $\hat{O}_{x}$

$$
\mathscr{D e t}\left(H^{*}\left(\mathscr{A}_{X, x}(\mathscr{F})\right)\right):=\operatorname{Det}\left(H^{0}\left(\mathscr{A}_{X, x}(\mathscr{F})\right)\right)-\mathscr{D e t}\left(H^{1}\left(\mathscr{A}_{X, x}(\mathscr{F})\right)\right) .
$$

Indeed, isomorphism (5-8) follows from Proposition 3.8 applied to the long exact sequence (decomposed into the short exact sequences) associated with the following exact sequence of complexes of length 2 for any nonzero free subsheaves $\mathscr{G} \subset \mathcal{H}$ of Frac $\hat{O}_{x}$ on the scheme $\operatorname{Spec} \hat{\hat{O}_{x}}$ :

$$
0 \longrightarrow \mathscr{A}_{X, x}(\mathscr{G}) \longrightarrow \mathscr{A}_{X, x}(\mathscr{H}) \longrightarrow \mathscr{A}_{X, x, 1}(\mathscr{H}) / \mathbb{A}_{X, x, 1}(\mathscr{G}) \longrightarrow 0,
$$

where the last complex consists only of the group placed in degree zero. Now we have 
$\operatorname{Det}\left(H^{*}\left(\mathscr{A}_{X, x}\left(d \hat{0}_{x}\right)\right)\right)-\operatorname{Det}\left(H^{*}\left(\mathscr{A}_{X, x}\left(\hat{\mathscr{O}}_{x}\right)\right)\right)$

$$
\simeq \operatorname{Hom}_{\mathscr{P P i c}} \mathbb{\mathbb { Z }}\left(\operatorname{Det}\left(H^{*}\left(\mathscr{A}_{X, x}\left(\hat{\mathrm{O}}_{x}\right)\right)\right), \operatorname{Det}\left(H^{*}\left(\mathscr{A}_{X, x}\left(d \hat{\mathrm{O}}_{x}\right)\right)\right)\right. \text {. }
$$

Multiplication by the element $d \in$ Frac $\hat{O}_{x}^{\times}$between adèle complexes $\mathscr{A}_{X, x}\left(\hat{\mathrm{O}}_{x}\right)$ and $\mathscr{A}_{X, x}\left(d \hat{O}_{x}\right)$ gives a natural isomorphism of $\mathscr{P} i c^{\mathbb{Z}}$-torsor from formula (5-9) to the trivial torsor $\mathscr{P} i c^{\mathbb{Z}}$.

Let $H$ be the subgroup of Frac $\hat{O}_{x}^{\times}$generated by the elements $f, g, h \in$ Frac $\hat{O}_{x}^{\times}$. Now we proceed as the proof of Weil reciprocity law (see Equation (5-1)), with the help of Lemma 4.9, Lemma 4.10, and Corollary 2.20. Then we obtain the following equality:

$$
\prod_{\mathbf{C} \ni x}\{f, g, h\}_{x, \mathbf{C}}=1 .
$$

Formula (5-5) follows from the last formula, since if a prime ideal $\mathbf{C}$ of height 1 in $\hat{O}_{x}$ is not a formal branch at $x$ of some irreducible curve $C$ on $X$, then for any element $d \in K_{x}^{\times}$we have $d \mathscr{O}_{x, \mathbf{C}}=\mathscr{O}_{x}, \mathbf{C}$. Hence, by formula (5-7), $\{f, g, h\}_{x, \mathbf{C}}=1$ for such $\mathbf{C}$ and any $f, g, h \in K_{x}^{\times}$.

Next we will prove formula (5-6). We construct the central extension $\mathscr{D e t}_{x, C}^{\prime}$ of the group $k(X)^{\times}$by the Picard groupoid $\mathscr{P} i c^{\mathbb{Z}}$ in the following way. We fix a point $x \in C$, and associate with the rings $B_{x} \subset K_{x, C}$ and with an element $d \in k(X)^{\times}$the following $\mathscr{P i c} c^{\mathbb{Z}}$-torsor:

$$
\operatorname{Det}\left(B_{x} \mid d B_{x}\right):=\operatorname{Det}\left(\frac{d B_{x}}{B_{x} \cap d B_{x}}\right)-\operatorname{Det}\left(\frac{B_{x}}{B_{x} \cap d B_{x}}\right) \text {. }
$$

(We used that $B_{x} / B_{x} \cap d B_{x}$ is a 1-Tate vector space over the field $k$.) By the formula which is analogous to formula (4-7) we obtain that the central extension Det $t_{x, C}^{\prime}$ is well defined. In a similar way we define the central extensions $\mathscr{D e t}_{C}^{\prime}$ and Det $t_{C \backslash\left\{x_{1}, \ldots, x_{l}\right\}}^{\prime}$ starting from the rings $\mathbb{A}_{X, C, 1}\left(\mathbb{O}_{X}\right) \subset \mathbb{A}_{X, C}$ and $\mathbb{A}_{X, C \backslash\left\{x_{1}, \ldots, x_{l}\right\}, 1}\left(\mathbb{O}_{X}\right) \subset$ $\mathbb{A}_{X, C \backslash\left\{x_{1}, \ldots, x_{l}\right\}}$, where $x_{1}, \ldots, x_{l}$ are some points on the curve $C$.

Let the group $H$ be generated in the group $k(X)^{\times}$by the elements $f, g, h \in$ $k(X)^{\times}$. For almost all points $x$ of the curve $C$ we have that the group $H$ preserves the subring $B_{x}$. Therefore form formula (5-10) we obtain that the central extension Det $t_{x, C}^{\prime}$ is isomorphic to the trivial one for almost all points $x$ of the curve $C$. Therefore for almost all points $x$ of the curve $C$ we have $C_{3}^{\mathscr{D} e t_{x, C}^{\prime}}(f, g, h)=1$.

We will prove that the central extension $\mathscr{D e t}_{x, C}^{\prime}$ is inverse (or dual) to the central extension $\mathscr{D e t}_{x, C}$, where the last central extension is constructed by formula (4-6) from the lattice $O_{x, C}$ in the 2-Tate vector space $K_{x, C}$. For any free subsheaf $\mathscr{F}_{F}$ of the constant sheaf Frac $\hat{O}_{x}$ on the scheme Spec $\hat{O}_{x}$ there is the following complex $\mathscr{A}_{X, C, x}(\mathscr{F})$ :

$$
\left(B_{x} \otimes_{\hat{\mathrm{O}}_{x}} \mathscr{F}\right) \oplus\left(\hat{\mathrm{O}}_{x, C} \otimes_{\hat{\mathrm{O}}_{x}} \mathscr{F}\right) \longrightarrow K_{x, C}
$$


We have canonically that $H^{*}\left(\mathscr{A}_{X, C, x}(\mathscr{F})\right)=H^{*}\left(U_{x},\left.\mathscr{F}\right|_{U_{x}}\right)$, where we recall $U_{x}=$ $\operatorname{Spec} \hat{O}_{x} \backslash x$ (see the proof of [Osipov 2005, Proposition 13]). Therefore the cohomology groups of complex $\mathscr{A}_{X, C, x}(\mathscr{F})$ are 1-Tate vector spaces. Hence, there is a canonical isomorphism between the following $\mathscr{P} i c^{\mathbb{Z}}$-torsors for any $d \in k(X)^{\times}$:

$$
\begin{aligned}
& \operatorname{Det}\left(B_{x} \mid d B_{x}\right)+\operatorname{Det}\left(\hat{\mathscr{O}}_{x, C} \mid d \hat{\widehat{O}}_{x, C}\right), \\
& \operatorname{Hom}_{\mathscr{P} i c^{\mathbb{Z}}}\left(\mathscr{D e t}\left(H^{*}\left(\mathscr{A}_{X, C, x}\left(\hat{\mathscr{O}}_{x}\right)\right)\right), \operatorname{Det}\left(H^{*}\left(\mathscr{A}_{X, C, x}\left(d \hat{\mathscr{O}}_{x}\right)\right)\right)\right) .
\end{aligned}
$$

Now multiplication by the element $d$ of adèle complexes gives a natural isomorphism from the last $\mathscr{P} i c^{\mathbb{Z}}$-torsor to the trivial one. Hence from Corollary 2.20 we have that

$$
C_{3}^{\mathscr{D} e t_{x, C}^{\prime}}(f, g, h)=C_{3}^{\mathscr{D} t_{x, C}^{\prime}}(f, g, h)^{-1}=\operatorname{Nm}_{k(x) / k}\{f, g, h\}_{x, C}^{-1}
$$

for $f, g, h \in k(X)^{\times}$.

Now the proof of formula (5-6) for elements $f, g, h \in k(X)^{\times}$follows by the same method as in the proof of formula (5-5), but we have to use the adèle ring $\mathbb{A}_{X, C}$ instead of the ring $\mathbb{A}_{X, x}$, and to use the central extension $\mathscr{D e t}_{C}^{\prime}$ instead of the central extension $\mathscr{D e t}_{x}$. We need only to prove that the central extension Det$_{C}^{\prime}$ constructed by the analog of formula (5-10) from the rings $\mathbb{A}_{X, C, 1}\left(0_{X}\right) \subset \mathbb{A}_{X, C}$ is isomorphic the trivial central extension. This follows if we consider the following $\mathscr{P i c} c^{\mathbb{Z}}$-torsors for $d \in k(X)^{\times}$

$$
\operatorname{Hom}_{\mathscr{P} i c^{\mathbb{Z}}}\left(\operatorname{Det}\left(H^{*}\left(\mathscr{A}_{X, C}\left(\mathscr{O}_{X}\right)\right)\right), \operatorname{Det}\left(H^{*}\left(\mathscr{A}_{X, C}\left(d \mathscr{O}_{X}\right)\right)\right)\right) \text {, }
$$

where

$$
\mathscr{D e t}\left(H^{*}\left(\mathscr{A}_{X, C}\left(d \mathscr{O}_{X}\right)\right):=\operatorname{Det}\left(H^{0}\left(\mathscr{A}_{X, C}\left(d \mathscr{O}_{X}\right)\right)-\operatorname{Det}\left(\tilde{H}^{1}\left(\mathscr{A}_{X, C}\left(d \mathscr{O}_{X}\right)\right) .\right.\right.\right.
$$

Multiplication by $d \in k(X)^{\times}$of adèle complexes gives the triviality of the $\mathscr{P} i c^{\mathbb{Z}}$ torsor (5-11). (See analogous reasonings earlier in the proof of this theorem.)

To obtain formula (5-6) for elements $f, g, h \in K_{C}^{\times}$we have to use that the field $k(X)$ is dense in the field $K_{C}$. Therefore for any element $f \in K_{C}^{\times}$there is an element $\tilde{f} \in k(X)^{\times}$such that $f=\tilde{f} m$, where the element $m$ is from the subgroup $1+m_{C}^{n}$ of the group $K_{C}^{\times}$for some $n \geq 1$, and $m_{C}$ is the maximal ideal of the valuation ring of discrete valuation field $K_{C}$. Then from formula (4-3) we have that $\{m, g, h\}_{x, \mathbf{C}}=1$ for any point $x \in C$, and any formal branch $\mathbf{C}$ of the curve $C$ at point $x$. Hence, from the trimultiplicativity of the two-dimensional tame symbol we obtain that

$$
\{f, g, h\}_{x, \mathbf{C}}=\{\tilde{f}, g, h\}_{x, \mathbf{C}} .
$$

Applying successively the same procedure to elements $g, h \in k_{C}^{\times}$we obtain

$$
\{f, g, h\}_{x, \mathbf{C}}=\{\tilde{f}, \tilde{g}, \tilde{h}\}_{x, \mathbf{C}},
$$


where $\tilde{f}, \tilde{g}, \tilde{h} \in k(X)^{\times}$, and any point $x \in C$, and $\mathbf{C}$ is any formal branch of the curve $C$ at point $x$.

Remark 5.4. For the proof of Parshin reciprocity laws we used "semilocal" adèle complexes of length 2 connected with either points or irreducible curves on an algebraic surface. But for the formulation of these reciprocity laws we used the rings $K_{x}$ and $K_{C}$ which appear from the "global" adèle complex of length 3 on an algebraic surface. It would be interesting to find direct connections between the "global" adèle complex and "semilocal" adèle complexes of an algebrac surface.

Remark 5.5. We have a symmetric monoidal functor from the Picard torsor $\mathscr{P i c}{ }^{\mathbb{Z}}$ to the Picard groupoid $\mathbb{Z}$ which sends every graded line to its grading element from $\mathbb{Z}$, where $\mathbb{Z}$ is considered as the groupoid with objects equal to $\mathbb{Z}$ and morphisms equal to identities morphisms. Under this functor a central extension of a group $G$ by a $\mathscr{P} i c^{\mathbb{Z}}$-torsor goes to the usual central of the group $G$ by the group $\mathbb{Z}$. In this way the map $v_{\mathbb{K}}$ for a two-dimensional local field $\mathbb{K}$ was obtained as the commutator of elements in this central extension in [Osipov 2005]. Also in this same reference the reciprocity laws for the map $v_{\mathbb{K}}$ were proved by the adèle complexes on an algebraic surface.

\section{Acknowledgments}

Osipov is grateful to L. Breen, M. Kapranov and A. N. Parshin for useful discussions on categorical approach to the two-dimensional tame symbol at various periods of time. Zhu thanks V. Kac for useful discussions.

\section{References}

[Arbarello et al. 1988] E. Arbarello, C. De Concini, V. G. Kac, and C. Procesi, "Moduli spaces of curves and representation theory", Comm. Math. Phys. 117:1 (1988), 1-36. MR 89i:14019 Zbl 0647.17010

[Arkhipov and Kremnizer 2010] S. Arkhipov and K. Kremnizer, "2-gerbes and 2-Tate spaces", pp. 23-35 in Arithmetic and geometry around quantization, edited by O. Ceyhan et al., Progr. Math. 279, Birkhäuser, Boston, MA, 2010. MR 2011g:22036 Zbl 05831590

[Baez and Neuchl 1996] J. C. Baez and M. Neuchl, "Higher-dimensional algebra, I: Braided monoidal 2-categories”, Adv. Math. 121:2 (1996), 196-244. MR 97h:18009

[Beilinson 1980] A. A. Beilinson, "Residues and adèles", Funktsional. Anal. i Prilozhen. 14:1 (1980), 44-45. In Russian; translated in Func. Anal. Appl. 14:1 (1980), 34-35. MR 81f:14010 Zbl 0509.14018

[Beilinson 1987] A. A. Beilinson, "How to glue perverse sheaves", pp. 42-51 in K-theory, arithmetic and geometry (Moscow, 1984-1986), edited by Y. I. Manin, Lecture Notes in Math. 1289, Springer, Berlin, 1987. MR 89b:14028 Zbl 0651.14009

[Beilinson et al. 2002] A. Beilinson, S. Bloch, and H. Esnault, " $\epsilon$-factors for Gauss-Manin determinants”, Mosc. Math. J. 2:3 (2002), 477-532. MR 2004m:14011 Zbl 1061.14010 
[Breen 1992] L. Breen, "Théorie de Schreier supérieure", Ann. Sci. École Norm. Sup. (4) 25:5 (1992), 465-514. MR 93k:18019

[Breen 1994] L. Breen, On the classification of 2-gerbes and 2-stacks, Astérisque 225, Société mathématique de France, Paris, 1994. MR 95m:18006 Zbl 0818.18005

[Breen 1999] L. Breen, "Monoidal categories and multiextensions", Compositio Math. 117:3 (1999), 295-335. MR 2000i:18009 Zbl 0932.18002

[Deligne 1973] P. Deligne, "La formule de dualité globale", pp. 481-587 in Théorie des topos et cohomologie étale des schémas (Séminaire de Géométrie Algébrique du Bois-Marie 1963-1964 = SGA 4), vol. 3, edited by M. Artin et al., Lecture Notes in Math. 305, Springer, Berlin, 1973. MR 50 \#7132 Zbl 0245.00002

[Deligne 1991] P. Deligne, "Le symbole modéré", Inst. Hautes Études Sci. Publ. Math. 73 (1991), 147-181. MR 93i:14030 Zbl 0749.14011

[Drinfeld 2006] V. Drinfeld, "Infinite-dimensional vector bundles in algebraic geometry: an introduction", pp. 263-304 in The unity of mathematics (Cambridge, MA, 2003), edited by P. Etingof et al., Progr. Math. 244, Birkhäuser, Boston, MA, 2006. MR 2007d:14038 Zbl 1108.14012

[Frenkel and Zhu 2008] E. Frenkel and X. Zhu, "Gerbal representations of double loop groups", preprint, 2008. arXiv 0810.1487

[Huber 1991] A. Huber, "On the Parshin-Beǔlinson adèles for schemes", Abh. Math. Sem. Univ. Hamburg 61 (1991), 249-273. MR 92k:14024

[Kapranov 2001] M. Kapranov, "Semiinfinite symmetric powers", 2001. arXiv math.AQ/0107089

[Kapranov and Voevodsky 1994] M. M. Kapranov and V. A. Voevodsky, "2-categories and Zamolodchikov tetrahedra equations", pp. 177-259 in Algebraic groups and their generalizations: quantum and infinite-dimensional methods (University Park, PA, 1991), edited by W. J. Haboush and B. J. Parshall, Proc. Sympos. Pure Math. 56, Amer. Math. Soc., Providence, 1994. MR 95f:18011 Zbl 0809.18006

[Kato 1986] K. Kato, "Milnor $K$-theory and the Chow group of zero cycles", pp. 241-253 in Applications of algebraic K-theory to algebraic geometry and number theory (Boulder, CO, 1983), edited by S. J. Bloch et al., Contemp. Math. 55, Amer. Math. Soc., Providence, 1986. MR 88c:14012 Zbl 0603.14009

[Osipov 2003] D. Osipov, "To the multidimensional tame symbol”, preprint 03-13, Humboldt University, Berlin, 2003, Available at http://edoc.hu-berlin.de/docviews/abstract.php?id=26204.

[Osipov 2005] D. V. Osipov, "Central extensions and reciprocity laws on algebraic surfaces", Mat. Sb. 196:10 (2005), 111-136. In Russian; translated in Sb. Math. 196:10 (2005), 1503-1527. MR 2007f:11071 Zbl 1177.14083 arXiv math.NT/0501155

[Osipov 2007] D. Osipov, "Adeles on $n$-dimensional schemes and categories $C_{n}$ ", Internat. J. Math. 18:3 (2007), 269-279. MR 2008b:14005 Zbl 1126.14004 arXiv math.AG/0509189

[Osipov 2008] D. V. Osipov, " $n$-dimensional local fields and adeles on $n$-dimensional schemes", pp. 131-164 in Surveys in contemporary mathematics, edited by N. Young and Y. Choi, London Math. Soc. Lecture Note Ser. 347, Cambridge Univ. Press, Cambridge, 2008. MR 2009b:14044 Zbl 1144.11078

[Parshin 1975] A. N. Parshin, "Class fields and algebraic K-theory", Uspehi Mat. Nauk 30:1 (181) (1975), 253-254. In Russian. MR 53 \#5537 Zbl 0302.14005

[Parshin 1976] A. N. Parshin, "On the arithmetic of two-dimensional schemes, I: Distributions and residues”, Izv. Akad. Nauk SSSR Ser. Mat. 40:4 (1976), 736-773. In Russian; translated in Math. USSR Izv. 10:4 (1976), 695-729. MR 54 \#7479 
[Parshin 1984] A. N. Parshin, "Local class field theory", pp. 143-170 in Algebraic geometry and its applications, Trudy Mat. Inst. Steklov. 165, 1984. In Russian; translated in Proc. Steklov Inst. Math. 1985, Issue 3, 157-185. MR 85m:11086 Zbl 0535.12013

[Quillen 1973] D. Quillen, "Higher algebraic K-theory. I", pp. 85-147 in Algebraic K-theory, I: Higher K-theories (Seattle, 1972), edited by H. Bass, Lecture Notes in Math. 341, Springer, Berlin, 1973. MR 49 \#2895 Zbl 0292.18004

[Serre 1988] J.-P. Serre, Algebraic groups and class fields, Graduate Texts in Mathematics 117, Springer, New York, 1988. MR 88i:14041 Zbl 0703.14001

[Tate 1968] J. Tate, "Residues of differentials on curves", Ann. Sci. École Norm. Sup. (4) 1 (1968), 149-159. MR 37 \#2756 Zbl 0159.22702

[Zhu 2009] X. Zhu, "The 2-group of linear auto-equivalences of an abelian category and its Lie 2-algebra", preprint, 2009. arXiv 0910.5699

Communicated by Michael Kapranov

Received 2010-02-27 Revised 2010-10-25 Accepted 2010-11-21

d_osipov@mi.ras.ru

Steklov Mathematical Institute, Gubkina str. 8, Moscow, 119991, Russia

xinwenz@math.harvard.edu Department of Mathematics, Harvard University, 1 Oxford Street, Cambridge, MA, 02138, United States 


\section{Algebra \& Number Theory}

www.jant.org

\section{EDITORS}

MANAGING EDITOR

Bjorn Poonen

Massachusetts Institute of Technology

Cambridge, USA

\author{
EDITORIAL BOARD CHAIR \\ David Eisenbud \\ University of California \\ Berkeley, USA
}

BOARD OF EDITORS

Georgia Benkart

Dave Benson

Richard E. Borcherds

John H. Coates

J-L. Colliot-Thélène

Brian D. Conrad

Hélène Esnault

Hubert Flenner

Edward Frenkel

Andrew Granville

Joseph Gubeladze

Ehud Hrushovski

Craig Huneke

Mikhail Kapranov

Yujiro Kawamata

János Kollár

Yuri Manin

Barry Mazur

Susan Montgomery
University of Wisconsin, Madison, USA

University of Aberdeen, Scotland

University of California, Berkeley, USA

University of Cambridge, UK

CNRS, Université Paris-Sud, France

University of Michigan, USA

Universität Duisburg-Essen, Germany

Ruhr-Universität, Germany

University of California, Berkeley, USA

Université de Montréal, Canada

San Francisco State University, USA

Hebrew University, Israel

University of Kansas, USA

Yale University, USA

University of Tokyo, Japan

Princeton University, USA

Northwestern University, USA

Harvard University, USA

University of Southern California, USA
Shigefumi Mori

Andrei Okounkov

Raman Parimala

Victor Reiner

Karl Rubin

Peter Sarnak

Michael Singer

Ronald Solomon

Vasudevan Srinivas

J. Toby Stafford

Bernd Sturmfels

Richard Taylor

Ravi Vakil

Michel van den Bergh

Marie-France Vignéras

Kei-Ichi Watanabe

Andrei Zelevinsky

Efim Zelmanov
RIMS, Kyoto University, Japan

Princeton University, USA

Emory University, USA

University of Minnesota, USA

University of California, Irvine, USA

Princeton University, USA

North Carolina State University, USA

Ohio State University, USA

Tata Inst. of Fund. Research, India

University of Michigan, USA

University of California, Berkeley, USA

Harvard University, USA

Stanford University, USA

Hasselt University, Belgium

Université Paris VII, France

Nihon University, Japan

Northeastern University, USA

University of California, San Diego, USA

\section{PRODUCTION}

contact@msp.org

Silvio Levy, Scientific Editor

Andrew Levy, Production Editor

See inside back cover or www.jant.org for submission instructions.

The subscription price for 2011 is US \$150/year for the electronic version, and \$210/year (+ \$35 shipping outside the US) for print and electronic. Subscriptions, requests for back issues from the last three years and changes of subscribers address should be sent to Mathematical Sciences Publishers, Department of Mathematics, University of California, Berkeley, CA 94720-3840, USA.

Algebra \& Number Theory (ISSN 1937-0652) at Mathematical Sciences Publishers, Department of Mathematics, University of California, Berkeley, CA 94720-3840 is published continuously online. Periodical rate postage paid at Berkeley, CA 94704, and additional mailing offices.

ANT peer review and production are managed by EditFLOw ${ }^{\mathrm{TM}}$ from Mathematical Sciences Publishers.

PUBLISHED BY

mathematical sciences publishers

http://msp.org/

A NON-PROFIT CORPORATION

Typeset in LATEX

Copyright $\odot 2011$ by Mathematical Sciences Publishers 


\section{Algebra \& Number Theory}

Volume $5 \quad$ No. $3 \quad 2011$

A categorical proof of the Parshin reciprocity laws on algebraic surfaces DENIS OSIPOV and XINWEN ZHU

Quantum differentiation and chain maps of bimodule complexes

ANNE V. SHEPLER and SARAH WITHERSPOON

Toric-friendly groups

MIKHAIL BOROVOI and ZINOVY REICHSTEIN

LuCHEZAR AVRAMOV, SRIKANTH B. IYENGAR and JOSEPH LIPMAN 